\title{
Les entreprises de création de sites web face à la rareté des compétences
}

The emerging Sector of Web Sites Creation and the Scarcity and the low

Visibility of Skills

\section{Yan Dalla Pria}

\section{OpenEdition}

\section{Journals}

Édition électronique

URL : http://journals.openedition.org/travailemploi/4834

DOI : 10.4000/travailemploi.4834

ISSN : 1775-416X

Éditeur

DARES - Ministère du Travail

Édition imprimée

Date de publication : 15 octobre 2006

Pagination : 7-18

ISSN : 0224-4365

\section{Référence électronique}

Yan Dalla Pria, "Les entreprises de création de sites web face à la rareté des compétences », Travail et Emploi [En ligne], 108 | octobre-décembre 2006, mis en ligne le 15 décembre 2008, consulté le 20 avril 2019. URL : http://journals.openedition.org/travailemploi/4834; DOI : 10.4000/ travailemploi.4834 


\section{T RAVAIL ET EMPLOI}

N 108 - Octobre-décembre 2006

Ministère

de l'emploi, de la cohésion sociale

et du logement

Direction de l'animation de la recherche,

des études et des statistiques 


\section{INDEX DE TRAVAIL ET EMPLOI}

L'index de l'ensemble des articles publiés dans la revue Travail et Emploi depuis sa création en 1979 est consultable sur le site Internet du Ministère de l'emploi, de la cohésion sociale et du logement dans la rubrique «Études et statistiques» (http://www.travail.gouv.fr/etudes/etudes_travail_emploi. html).

Cet index propose une recherche d'articles par grandes rubriques thématiques (index matières) et par auteurs (index auteurs, organisé par lettre initiale du patronyme des auteurs publiés). La consultation est proposée en téléchargement au format Word permettant ainsi d'enregistrer et d'imprimer facilement le contenu de l'index.

L'index «matières» et «auteurs» est mis à jour lors de la sortie de chaque nouveau numéro.

(c) La Documentation française - Paris, 2006

C C Ministère de l'emploi, de la cohésion sociale et du logement - Paris, 2006

«En application de la loi du 11 mars 1957 (art. 41) et du code de la propriété intellectuelle du 1er-juillet 1992, complétés par la loi du 3 janvier 1995, toute reproduction partielle ou totale à usage collectif de la présente publication est strictement interdite sans autorisation expresse de l'éditeur.

Il est rappelé à cet égard que l'usage abusif et collectif de la photocopie met en danger l'équilibre économique des circuits du livre». 
MARCHÉ DU TRAVAIL

Les entreprises de création de sites web face à la rareté des compétences

Yan Dalla Pria

\section{INTERNATIONAL}

Les catégories d'emploi dans la société japonaise

Kurumi Sugita

Les fusions théâtrales dans les nouveaux Länder:

héritage culturel, fardeau financier

Laure de Verdalle

\section{MARCHE DE L'EMPLOI}

La relation innovation - emploi dans les services

Faridah Djellal, Faïz Gallouj

Service public et sous-traitance :

la France au regard des expériences australiennes, britanniques et néerlandaises 



\section{MARCHÉ DU TRAVAIL}

\section{Les entreprises de création de sites web face à la rareté des compétences Yan Dalla Pria}

La rareté et la faible visibilité des compétences sur le marché du travail du secteur émergent de la création de sites web ont exercé une contrainte forte sur le fonctionnement des organisations spécialisées dans ces activités en France et aux États-Unis à la fin des années 1990. En prenant appui sur le cas de la start-up parisienne Babylone Connection, l'article propose de mettre en évidence la richesse et l'originalité des stratégies développées par les organisations pionnières de ce secteur d'activités en matière de recrutement, de fidélisation du personnel et d'organisation interne des activités productives, afin de s'adapter à cette conjoncture très exceptionnelle du marché du travail.

\section{INTERNATIONAL}

\section{Les catégories d'emploi dans la société japonaise Kurumi Sugita}

L'article analyse les catégories d'emploi telles qu'elles sont pratiquées dans le système statistique japonais. À partir de l'analyse des questionnaires des enquêtes principales sur l'emploi, l'auteure met en évidence des stratifications de significations successives à travers l'histoire, ce qui rend flou le contour de certaines catégories d'emploi. Leur caractère équivoque proviendrait du fait que la dénomination ordinaire est utilisée dans le système d'observation statistique ou que le système de classification savant se base sur les pratiques des entreprises. Toutefois, en examinant les changements récents dans les questionnaires, l'auteur met en évidence des tensions entre deux langages différents, appartenant aux deux champs, celui de la loi (introduction très récente) et celui de l'entreprise. Ces tensions semblent augmenter dans la situation actuelle de crise où l'État tente de revenir devant la scène afin de se charger des fonctions délaissées par les entreprises défaillantes.

\section{Les fusions théâtrales dans les nouveaux Länder: héritage culturel, fardeau financier Laure de Verdalle}

En 1990, au moment de la réunification, les décalages qui séparent le paysage théâtral de la RFA et celui de la RDA sont évidents. L'application rapide des règles ouest-allemandes sur le tissu théâtral est-allemand va donc avoir des conséquences fortes. La restructuration du paysage théâtral des nouveaux Länder se traduit par l'organisation de fusions regroupant différents théâtres publics. L'article s'intéresse aux modalités de mise en œuvre de ces fusions, qui exacerbent les rivalités entre municipalités et qui bousculent l'organisation du travail théâtral. Ce processus de rationalisation du tissu théâtral est-allemand constitue un exemple intéressant pour étudier l'application des modèles gestionnaires à la sphère culturelle. Les difficultés rencontrées par les structures concernées, les tâtonnements des différents acteurs en présence ainsi que le caractère inéluctable des restrictions budgétaires mises en œuvre conduisent l'auteure à interroger l'hypothèse d'un possible effet retour de la réunification qui semble annoncer une remise en cause du modèle ouest-allemand de relations professionnelles.

\section{MARCHÉ DE L'EMPLOI}

\section{La relation emploi - innovation dans les services Faridah Djellal, Faïz Gallouj}

Cet article est consacré à la difficile question de la relation entre l'innovation et l'emploi dans les services. Il propose une relecture, à la lumière de la question de l'emploi, des travaux consacrés à l'innovation dans les services. Il s'agit d'évaluer dans quelle mesure et sous quelle forme cette question y est implicitement ou explicitement présente ou mérite d'y être introduite. Ce projet constitue à la fois un bilan de la littérature nationale et internationale et un agenda de recherche. On explorera ainsi successivement les deux thèmes suivants : les approches technologistes et la question de l'emploi; la question de l'emploi dans les approches servicielles.

\section{Service public et sous-traitance: la France au regard des expériences australiennes, britanniques et néerlandaises}

Céline Gratadour, Michèle Mansuy

La sous-traitance des services d'accompagnement des chômeurs est une tendance générale. Pour éclairer la situation de la France, où cette possibilité a été récemment étendue sous l'égide de l'ANPE et de l'UNEDIC, cet article propose d'utiliser l'exemple des pays qui ont poussé le plus loin la logique d'intervention des opérateurs privés, l'Australie, les Pays-Bas et à un moindre degré le Royaume-Uni. Dans un premier temps, il examine les fonctions du SPE qui sont sous-traitées et les formes d'implication possibles des prestataires privés. Dans une deuxième partie, à la lumière de la théorie de l'agence, sont exposés les risques associés à la sous-traitance de l'accompagnement des chômeurs, essentiellement un problème d'aléa moral lié à l'asymétrie d'information que les prestataires privés peuvent utiliser en adoptant des comportements opportunistes. Pour le résoudre, l'acteur public principal doit mettre en place des systèmes de gouvernance adaptés, ex ante et ex post. 


\section{Revue}

internationale

du Travail

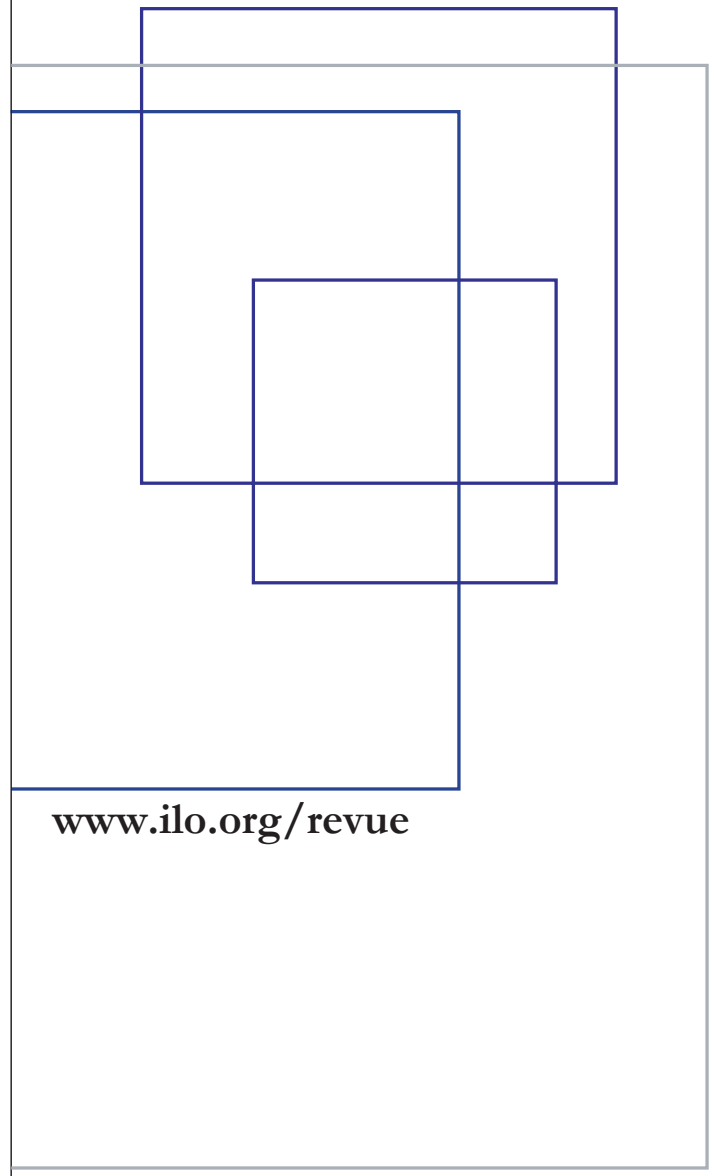

Vol. 145 (2006), no. 1-2

\section{Numéro spécial: migrations}

Potentiel des programmes de migration temporaire dans l'organisation de migrations internationales

M. RUHS

La mondialisation du marché du travail des personnels de santé

P. F. CLARK, J. B. STEWART et D. A. CLARK

La chasse à la matière grise: Les stratégies de la Chine pour faire revenir les cerveaux

D. ZWEIG

Effets de l'immigration sur le marché du travail: Le cas de l'Italie

A. VENTURINI et C. VILLOSIO

\section{Perspectives}

L'internationalisation de l'emploi: Un défi pour une mondialisatin juste?

La nouvelle convention maritime:

Un instrument novateur

Livres

Abonnement à l'édition imprimée et accès à l'édition électronique (2006):

Institutions: 1 an: 180 fr. suisses; 120 euros. 2 ans: 290 fr. suisses; 190 euros.

Particuliers: 1 an: 90 fr. suisses; 60 euros. 2 ans: 145 fr. suisses; 95 euros. Edition électronique seulement: un an: 100 fr. suisses; 65 euros.

Publications du BIT, Bureau international du Travail, CH-1211 Genève 22, Suisse Fax: (41-22) 799.69.38; Tél.: 799.78.28; E-mail: pubvente@ilo.org 


\title{
Les entreprises de création de sites web face à la rareté des compétences
}

\author{
Yan Dalla Pria (*)
}

La rareté et la faible visibilité des compétences sur le marché du travail du secteur émergent de la création de sites web ont exercé une contrainte forte sur le fonctionnement des organisations qui se sont spécialisées dans ces activités à la fin des années 1990. L'article décrit les spécificités de ce type de situation: pénurie de main-d'œuvre qualifiée, forte rotation du personnel, absence des signaux classiques de la compétence, marché faiblement régulé, faible implantation syndicale. Puis, en s'appuyant sur le cas de la start-up parisienne Babylone Connection, il met en évidence la richesse et l'originalité des stratégies développées par cette organisation pionnière en matière de recrutement, de fidélisation du personnel et d'organisation interne des activités productives. Le modèle babylonien est caractérisé par une sélectivité forte, avec des accents «libertaires » et communautaires qui tendent à fidéliser les salariés; des organisations spécifiques, comme le travail en binômes, ou le staffing, permettent de cerner les niveaux individuels de compétence, renforcées ensuite par un double système de réseaux d'excellence pour les meilleurs et de formation pour les autres, de façon à pallier les contraintes du marché et à faire face à la double problématique de rareté des ressources humaines et d'incertitude de départ sur leur qualité.

Nouveau savoir-faire, nouveau procédé, nouvelle technologie: ces petites révolutions sont autant d'occasions de remise en question des compétences valorisées précédemment. Parfois, les nouvelles compétences requises sont aisées à acquérir et l'ajustement est rapide. D'autres fois, l'émergence de nouvelles compétences clés est anticipée et préparée par les acteurs du secteur. Cependant, il arrive que les évolutions en question soient émergentes, peu prévisibles, et qu'elles impliquent la maîtrise de compétences radicalement nouvelles, difficiles à acquérir sur le marché du travail dans les premiers temps (introduction de la Production et Conception Assistée par Ordinateur - PAO et CAO - dans l'industrie automobile, du Personal Computer - PC - dans les organisations, etc.). Ces situations ne sont certes pas fréquentes puisqu'elles sont initiées par des changements de savoir-faire ou de technologie majeurs et donc peu communs. Mais en dépit de leur marginalité, elles soulèvent une problématique plus large: comment les entreprises réagissent-elles lorsque le marché du travail de leur secteur d'activité est caractérisé par des compétences à la fois rares et difficilement identifiables? Nous nous proposons d'étudier cette question dans le cas des entreprises de création de site web.

\footnotetext{
* Maître de conférence à l’Université Paris-Nanterre, e-mail: y.dallapria@cso.cnrs.fr

Je remercie vivement Christine Musselin, Pierre François, Denis Segrestin, Erhard Friedberg et Michel Lallement pour l'aide précieuse qu'ils m'ont apportée lors de la réalisation de cet article.
}

Si elle n'est pas nouvelle en sciences économiques (théorie du signal : SPENCE, 1973 ; phénomènes de sélection adverse: AKerlof 1970, WeISS, 1980; règle du up or out: O'Flaherty, Siow, 1995) cette problématique n'a jamais été traitée en profondeur par la sociologie. En dépit de son abondance, la littérature sociologique relative au concept de compétence s'est en effet organisée autour de deux thématiques majeures, négligeant de ce fait l'étude de situations et de problématiques plus marginales. La première, assurément la plus pertinente dans le cas qui nous intéresse, est relative à l'évaluation des compétences lors des procédures de recrutement (EyMARD-Duvernay, Marchal, 1997, 2000 ; Bureau, Marchal, 2005). À partir de l'étude de situations empiriques diverses, ces travaux d'inspiration institutionnaliste visent à appréhender les dispositifs permettant la formation des jugements portés sur la compétence des candidats à un recrutement. Ils montrent notamment pour ce faire que les modes de jugement sur les compétences peuvent varier selon les conventions retenues par les recruteurs, variables d'une entreprise à l'autre. Mais si ces travaux apportent un éclairage intéressant sur les dispositifs de recrutement, ils n'abordent pas explicitement la question des conséquences d'une conjoncture du marché du travail aussi atypique que celle que nous proposons d'étudier sur les pratiques de recrutement, et encore moins sur l'organisation ultérieure des activités productives en interne.

La deuxième thématique majeure a trait à la gestion des compétences et à l'émergence postulée 
d'un «modèle de la compétence» (ZARIFIAN, 1988)(1). Si une partie des travaux rattachés à ce courant thématique a abordé la notion de compétence dans sa globalité (ZARIFIAN, 2001 ; Le BOTERF, 2000), de nombreux autres se sont focalisés sur la question de la filiation et des similitudes éventuelles existant entre le modèle de la compétence et celui de la qualification (PARADEISE, LiCHTENBERGER, 2001; Oiry, D'Iribarne, 2001). Parallèlement, la notion de compétence a également donné lieu à diverses approches critiques amenant par exemple à considérer le modèle de la compétence comme un instrument de domination managériale (DUGUÉ, 1994) ou aboutissant à une individualisation croissante en matière de gestion des ressources humaines et à un isolement du salarié désormais seul face à l'entreprise (Courpasson, Livian, 1991) (2). On soulignera enfin que le concept de compétence est désigné par Boltanski, ChiAPEllo (1999) comme l'une des caractéristiques marquantes du «nouvel esprit du capitalisme» en ce sens qu'il confère une valeur économique aux individus eux-mêmes et non plus seulement à leur travail. Mais en dépit de son abondance, ce deuxième courant de littérature sociologique demeure cantonné à des problématiques postulant l'existence d'une compétence et la possibilité de l'identifier.

Qu'en est-il alors des situations évoquées précédemment dans lesquelles la compétence est tout à la fois rare et difficilement identifiable pour une entreprise en phase de recrutement? Nous nous proposons dans cet article de traiter cette problématique en nous appuyant sur le cas du secteur économique de la création de sites web, né entre 1994 et 1997 aux États-Unis puis entre 1996 et 1999 en France consécutivement à l'émergence d'une innovation technologique: 1'Internet. Nous nous intéresserons pour ce faire au cas de la première génération d'entreprises qui s'est lancée sur ce secteur d'activité: les web agencies. Nous montrerons plus précisément comment ces start-up ont géré leur recrutement et leur fonctionnement interne dans un contexte économique de rareté de la main-d'œuvre qualifiée.

(1) Le concept sociologique de compétence a émergé à la fin des années 1980 en réaction à celui de qualification. Alors que le concept de qualification repose sur l'idée d'un poste défini pour lequel une personne possèderait ou non les aptitudes nécessaires, celui de compétence amène à considérer l'ensemble des connaissances, pratiques et attitudes, éventuellement évolutives et extensibles à d'autres métiers, d'un individu afin de définir la fonction qu'il pourrait occuper.

(2) Cette polémique autour de la signification du passage au modèle de la compétence a été mise en exergue par la revue Gérer et Comprendre (décembre 2000), qui a invité deux sociologues à confronter leurs vues sur la question : là où J-P. DuRAND dénonce «le dernier avatar en date du capitalisme le plus classique», P. ZARIFIAN entrevoit une rupture avec le taylorisme et un «retour du travail dans le travailleur».
Dans un premier temps, nous reviendrons sur la situation du marché du travail du secteur de l'Internet à Paris («Silicon Sentier») et à New York («Silicon Alley») à l'époque où sont apparues les premières web agencies. Nous montrerons notamment que ces start-up ont dû faire face au milieu des années 1990 à une situation aussi délicate qu'inhabituelle sur le marché du travail: rareté des compétences, absence de signaux clairs (pas de formations, de diplômes, etc.), abus en tous genres, absence de régulations collectives... Ces constats nous permettront de justifier l'utilisation du terme de «compétence» plutôt que celui de «qualification» pour le secteur d'activité considéré. Dans un deuxième temps, nous étudierons les stratégies développées à cette période par les web agencies pour faire face à la pénurie de main-d'œuvre qualifiée et à la difficile identification des compétences. Nous évoquerons pour ce faire le cas d'une web agency, nommée Babylone Connection pour des raisons de confidentialité, dont nous présenterons les stratégies de recrutement, de fidélisation du personnel puis d'organisation interne du travail.

\section{Encadré 1 \\ Méthodologie}

L'analyse des marchés du travail du Silicon Sentier parisien et de la Silicon Alley new-yorkaise se fonde sur des données collectées par entretiens auprès d'un échantillon représentatif des acteurs de ces districts (dirigeants de start-up et de grandes entreprises, capitaux-risqueurs, recruteurs, journalistes, pouvoirs publics, etc.). Ces entretiens individuels semi-directifs (une centaine environ) ont été réalisés pour moitié dans le Silicon Sentier de mars à septembre 2001 et pour moitié dans la Silicon Alley de novembre 2002 à février 2003. L'ouvrage de KAIT et WEISS (2001), qui rassemble de nombreux témoignages portant sur l'histoire de la Silicon Alley, a constitué une source complémentaire de données secondaires.

L'étude du fonctionnement des entreprises de création de site web repose sur une monographie d'entreprise rédigée à partir d'une soixantaine d'entretiens individuels semi-directifs réalisés dans une web agency parisienne (désignée par le pseudonyme de Babylone Connection pour des raisons d'anonymat). Ces entretiens ont été effectués de mai à septembre 2000 dans le cadre d'un mémoire de DEA de sociologie des organisations (IEP de Paris) et ont ensuite été analysés selon la méthode de l'analyse stratégique des organisations (Crozier, Friedberg, 1977). La représentativité des constats issus de cette monographie a été validée grâce aux entretiens réalisés ultérieurement dans d'autres start-up du Silicon Sentier et de la Silicon Alley. 


\section{Un marché du travail émergent}

L'apparition puis la médiatisation de l'Internet a incité un nombre croissant d'entreprises traditionnelles à se doter d'un site web, dans un contexte d'insuffisance de main-d'œuvre, d'opacité des niveaux de compétence et d'absence de régulation. Ne maîtrisant pas les compétences nécessaires, elles se sont tournées vers de jeunes start-up spécialisées dans cette activité: les web agencies. Ainsi, à partir de 1994 aux États-Unis et de 1996 en France, l'industrie du site web a profité de cette manne financière pour s'épanouir.

\section{La pénurie de main-d'œuvre qualifiée}

Mais, en dépit de cette conjoncture économique exceptionnellement favorable, l'expansion des web agencies a été freinée par une contrainte forte: la rareté de la main-d'œuvre maîtrisant les compétences nécessaires à la réalisation d'un site web (web design, codage HTML, développement informatique, etc.). Ainsi, dans le Silicon Sentier, «il y a eu un boom extraordinaire de création de start-up au début, d'où un fort besoin de maind'œuvre compétente dans un contexte où la force de travail était faible» (journaliste, Paris). Aussi, «à cette période, quand des profils techniciens se présentaient, on ne faisait pas trop la fine bouche car ils étaient rares» (dirigeant de start-up, Paris). En outre, cette conjoncture particulière a amené les web agencies et bien d'autres start-up à se trouver en situation de concurrence pour le recrutement: «Les PDG ne voulaient pas envoyer leurs employés représenter l'entreprise dans les First Tuesday(3), car ils étaient débauchés par d'autres PDG. C'était l'aspect volatile de la main-d'œuvre d'ingénieurs en développement, de développeurs, de web designers et de programmeurs» (journaliste, Paris).

À la même période, la situation du marché du travail de l'Internet dans la Silicon Alley newyorkaise présente des caractéristiques similaires: "Quand on a commencé à croître, on a dû accepter des CV pas terribles car tous les profils étaient difficiles à trouver» (dirigeant de start-up, New York). Les entreprises doivent faire face à l'existence d'un fort taux de rotation du personnel: «Le marché du travail était tellement tendu: les gens passaient d'une entreprise à l'autre !» (journaliste, New York), ainsi qu'à des comportements déloyaux de la part de startup concurrentes: «Les dirigeants d'entreprises étaient consternés car il n'y avait pas de loyauté. Les entreprises tentaient de débaucher les gens d'autres entreprises. Il y avait tellement de soirées que la proximité était grande: c'était très facile d'être présenté à d'autres $\mathrm{CEO} »$ (recruteur, New York).

(3) Rencontres entre financeurs et porteurs de projet organisées tous les premiers mardis du mois pendant les années fastes de la net économie.

\section{L'absence des signaux classiques de la compétence}

Pourcertains postes, la pénurie évoquée précédemment est simplement liée à l'explosion des besoins des entreprises (informaticiens notamment). Pour d'autres, un autre phénomène complique les choses: l'absence de «signaux (4)» (SPENCE, 1973) crédibles permettant d'identifier la compétence. Ainsi, les postes de codeur HTML ou de web designer par exemple requièrent des aptitudes nouvelles et ne correspondent à cette période à aucune formation reconnue (5). À cet égard, Fondeur et Sauviat (2002) ont montré qu'aujourd'hui encore, $44 \%$ des individus évoluant dans le secteur de l'Internet ne possédaient pas de diplôme ou avaient un niveau d'étude inférieur à $\mathrm{Bac}+2$, à la différence de ceux évoluant dans les autres métiers tertiaires des NTIC (conseil, édition ou SSII), très fortement qualifiés. De même, les sources d'information internes au secteur sont très rares ou imparfaites: à l'époque, les associations professionnelles du type Syntec informatique n'ont encore que peu développé leurs activités multimédias, les magazines en ligne ou portails spécialisés sur l'Internet naissent à peine (O1net. a été lancé en 1998 et le Journal du Net en 1999), etc.

Dans ce contexte, confrontés à la difficulté de devoir évaluer par eux-mêmes les talents d'autodidactes prétendant maîtriser telle ou telle compétence, les recruteurs ont dû se rabattre sur d'autres signaux. L'ancienneté relative dans le secteur d'activité est alors devenue le principal critère pris en compte: «C'était incontrôlé, si quelqu'un avait un an d'expérience on line, sa valeur sur le marché était énorme !» (recruteur, New York). Mais de manière générale, chaque dirigeant a géré son recrutement à sa manière avec plus ou moins de succès: «Je demandais simplement aux gens quel genre de musique ils aimaient, je ne faisais pas de test de personnalité» (dirigeant de start-up, New York), «j'ai d'abord recruté mon cousin, puis ça s'est fait par cooptation: c'est un moyen de créer une valeur de confiance partagée.» (dirigeant de start-up, Paris), «on en a recruté deux par une annonce dans [Magazine]. L'annonce disait: "fatigués, cheveux gras, rejoignez [notre start-up]" car il y avait une pub pour un shampoing qui disait la même chose à côté. On a eu des réponses de game designers passionnés: ils sont délirants et excellents.» (dirigeant de start-up, Paris).

(4) «Un signal est un attribut manipulable ou une activité qui transmet de l'information» (SPENCE, 1974).

(5) Cette absence de signaux fiables ne concerne pas les ingénieurs et informaticiens issus d'écoles anciennes dont le niveau des formations est bien connu. À cette époque, les start-up ont recruté nombre de ces jeunes diplômés et la difficulté qu'elles ont rencontrée a résidé dans leur rareté et non dans l'incertitude sur leur niveau de compétence. 
Mais en dépit de ces «recettes» personnelles, cette période a donné lieu à bien des abus, à Paris comme à New York, dont les jeunes start-up ont eu à assumer les conséquences (6). La conjoncture a d'abord permis à des gens peu compétents d'être recrutés: «Tu ne montrais même pas un $\mathrm{CV}$, tu n'avais rien à prouver ! Je demandais à être directeur de création associé avec seulement un ou deux ans d'expérience !!! Il fallait juste que tu sois très sûr de toi» (employée de start-up, New York), «notre chef de projet n'était pas un king d'Internet. On a galéré pour le trouver. Mais il crédibilisait ce projet et était indispensable pour lever des fonds car les financeurs rencontrés n'étaient pas assez bons pour voir qu'il n'était pas top» (dirigeant de start-up, Paris). D'autre part, on a assisté à une inflation des salaires, dont ont bénéficié les employés mais qui a nui au développement des start-up elles-mêmes: «On ne pouvait pas faire grand-chose: des gens normalement payés $50000 \$$ voulaient $70000 \$$.» (dirigeant de start-up, New York), «mon salaire a beaucoup augmenté en quelques mois en 19951996. Tu n'avais qu'à faire savoir que tu savais faire les choses.» (employée de start-up, New York), «il y avait des types qui disaient $\mathrm{OK}$ pendant l'entretien puis demandaient $40 \%$ de plus de salaire ensuite» (dirigeant de start-up, Paris).

\section{Des marchés du travail “faiblement régulés "}

On ne s'étonnera pas de trouver à New York un marché du travail des NTIC caractérisé par une très faible implantation des syndicats, une souplesse extrême du droit du travail et des salaires fluctuant librement au gré de l'offre et de la demande. En revanche, la même faiblesse des régulations sur le marché du travail des NTIC à Paris est plus surprenante dans un pays où le droit du travail est sensiblement plus développé. Cette situation se manifeste d'abord par la très faible implantation des syndicats dans les entreprises NTIC. Selon FondEUR et SAuviat (2002), près de $85 \%$ des employés du secteur n'ont jamais eu de contact autre qu'anecdotique avec un syndicat. D'autre part, les métiers et le fonctionnement des entreprises de la net économie s'avèrent particulièrement inadaptés aux modes d'action d'organisations professionnelles telles que la fédération Syntec, à laquelle sont rattachées les web agencies: «La convention Syntec Informatique régit les trois quarts du chiffre d'affaires de l'informatique français mais n'est pas adaptée à la nouvelle économie en raison des 35 heures et de la diversité des métiers» (association parisienne de start-up) ou encore "Syntec est une approche sectorielle alors que dans les métiers d'Internet, il y a de grandes différences. Du coup, on nomme des choses nouvelles de la net économie avec des

(6) Cf. Riou (2001) sur ce thème. termes anciens et c'est problématique car les statistiques rament.» (agent du ministère de l'Économie, des Finances et de l'Industrie). Cette idée est illustrée par LALLEMENT (2003) qui montre comment il a fallu attendre 1999 en France et les négociations sur la réduction du temps de travail (lois Aubry) pour que la fédération Syntec reprenne la main en proposant des éclaircissements sur les métiers du secteur NTIC: redéfinition du statut de cadre, distinction entre «vrais» et «faux» ingénieurs, etc.

Dans ces conditions, le marché du travail des web agencies (et des start-up Internet en général) à Paris comme à New York peut être considéré pendant la période qui nous intéresse comme «externe» (KerR, 1954), c'est-à-dire très faiblement organisé et proche du modèle économique classique de la concurrence pure et parfaite. L'absence de syndicalisme, le faible niveau de qualification de nombreux salariés, la libre circulation des individus au gré de l'évolution des salaires sont en effet caractéristiques de ce type de marché. En ce sens, le marché du travail de l'Internet se distinguait à cette période des autres marchés du travail du secteur high tech, caractérisés par l'existence de niveaux de qualification élevés et standardisés et donc plus proches du marché «professionnel», voire «interne» lorsque des compétences spécifiques entravent la mobilité externe. Certes, cette distinction, surtout adaptée au monde industriel, est aujourd'hui quelque peu datée et artificielle (LEFRESNE, 2002). Toutefois, elle conserve le mérite de caractériser les marchés du travail qui nous intéressent et de mettre en exergue l'existence dans le secteur tertiaire des NTIC d'employés faiblement qualifiés, évoluant sur des postes à faible qualification (HTMListes et infographistes notamment).

En conclusion, les marchés du travail parisien et new-yorkais de l'industrie du site web étaient relativement comparables dans la seconde moitié des années 1990. Ils étaient caractérisés par une pénurie de main-d'œuvre, une difficulté à identifier les niveaux de compétence et une absence de régulations collectives. Autant d'éléments qui ont contraint les recruteurs et dirigeants des web agencies et autres start-up à imaginer des stratégies originales de recrutement, de fidélisation du personnel et d'organisation interne du travail. Nous allons maintenant entrer dans le détail de ces stratégies au travers du cas d'une web agency parisienne.

\section{Le cas de Babylone Connection}

Nous étudierons cette entreprise sous l'angle de ses stratégies de recrutement et d'organisation du travail et confronterons ses pratiques avec celles d'autres cas de web agencies traités dans la littérature. 


\section{Présentation de l’entreprise}

Depuis sa création en 1995, la web agency Babylone Connection s'est développée lentement jusqu'en 1999 avant de connaître une augmentation importante de son chiffre d'affaires (27 millions de francs en 1999) et de son personnel (130 personnes en juillet 2000). Financièrement indépendante, l'entreprise prépare lors de la réalisation de l'étude son introduction en Bourse afin de disposer des capitaux nécessaires à son expansion. À terme, son objectif est de développer ses activités à l'étranger afin de devenir un leader européen dans son secteur. Ces ambitions de développement international rapide soutenu par une levée de fonds sur le marché bousier constituaient, avant l'éclatement de la «bulle Internet», une norme plus ou moins explicite de croissance pour les start-up.

L'organisation interne de Babylone Connection est structurée autour de départements fonctionnels appelés «studios». Chaque studio est dirigé par un manager de studio et regroupe des individus ayant des métiers proches. Le rôle du manager de studio est de gérer l'évolution de carrière des individus de son studio et de décider sur quel projet ils seront affectés (staffing).

La procédure standard de réalisation d'un site compte six étapes (proposition, conseil, définition, conception, production et mise à jour) impliquant chacune des studios différents. Les observations mobilisées dans ce papier pour décrire l'organisation interne du travail sont toutes tirées de la phase de production, riche en enseignements en raison de la densité des relations auxquelles elle donne lieu. Cette étape, généralement la plus longue (jusqu'à plusieurs mois), est marquée par la réalisation concrète du site (passage du papier à l'informatique) et implique un nombre important de personnes. Elle voit la création d'équipes projet constituées d'un chef de projet et de représentants de trois corps de métier techniques («professionnels»): infographistes, HTMListes et codeurs informatique. Ces derniers travaillent généralement beaucoup, pour un salaire parfois modéré, et effectuent des tâches relativement répétitives requérant peu de créativité. À l'exception de quelques «stars locales», ils peuvent donc être considérés comme les «ouvriers spécialisés (OS) du web». On soulignera enfin que les infographistes et les codeurs informatiques sont supervisés par le directeur artistique et le concepteur informatique ayant précédemment réalisé l'identité visuelle et l'architecture informatique du site respectivement.

Babylone Connection présente donc sur le papier une structure fonctionnelle relativement classique. Pour autant, cette structure ne dit rien du fonctionnement interne de l'entreprise. Dans la suite de cet article, nous chercherons à appréhender plus spécifiquement ce fonctionnement interne en mettant au jour les stratégies développées par l'entreprise pour répondre aux contraintes imposées par la conjoncture particulière de son marché du travail.

\section{Comment recruter des gens compétents sur un marché du travail émergent et les garder?}

La période de réalisation de l'étude (Printemps 2000) a coïncidé avec l'explosion de la bulle Internet. Pour autant, le retournement de l'activité économique de l'entreprise a été moins brutal que celui des marchés financiers et celle-ci était encore en pleine croissance à cette époque. Dans le contexte de main-d'œuvre rare évoqué précédemment et en l'absence de formations clairement reconnues aux nouveaux métiers du web (7), Babylone Connection, à l'image de la majorité des entreprises de l'Internet, connaissait des difficultés pour recruter des individus compétents mais également pour conserver les individus précédemment recrutés et formés, fortement sollicités par la concurrence. La variété des stratégies déployées autour de ces deux problématiques atteste de l'importance de cet enjeu pour l'entreprise.

\section{Les méthodes de recrutement: pénurie de main-d'ouvre et sélection adverse}

En réaction à la pénurie de main-d'œuvre, Babylone Connection a tout d'abord mis en place un système de cooptation en plus des offres d'emplois diffusées dans la presse et sur le web. Ce système prévoit l'octroi d'une prime de $5000 \mathrm{FF}$ à tout individu de Babylone étant à l'origine d'un recrutement. Cette pratique, qui incite les «babyloniens» à diffuser activement les offres d'emploi dans leur réseau personnel, est de nature à accroître les opportunités de recrutement pour l'entreprise: les contacts personnels constituent en effet pour un individu en recherche d'emploi le vecteur d'information le plus efficace sur les emplois nouvellement créés (GranovetTer, 1974). Ce système assure en outre une certaine homogénéité dans la population babylonienne.

Ensuite, la start-up recourt à diverses recettes classiques visant à limiter les problèmes de sélection adverse (8) (AKERLOF, 1970) liés à la difficulté à évaluer le niveau de compétence des personnes recrutées. En premier lieu, Babylone recourt fréquemment aux stages et aux contrats à durée déterminée et procède à un renouvellement quasi systématique voire abusif de la période d'essai: «[Direction] m'a proposé une troisième période d'essai.» (HTMListe).

(7) À cette période, les formations universitaires ou professionnelles aux métiers du web se développaient mais leur émergence récente ne permettait pas d'avoir une bonne visibilité sur leur qualité.

(8) Le problème de la sélection adverse apparaît lorsque l'une des parties possède une information privée ou cachée. Cf. l'exemple classique du marché des voitures d'occasion, lemons, étudié par AKERLOF (1970). 
Parallèlement, les épreuves de recrutement sont assorties lorsque cela est possible d'une validation des compétences techniques des candidats. Un test a par exemple été créé par un HTMListe expérimenté et reconnu afin de s'assurer des aptitudes des candidats à un recrutement dans ce métier: «Je m'occupe de l'embauche des nouveaux HTMListes après l'entretien avec les ressources humaines. Le test que j'ai élaboré consiste à leur donner une page à monter. On est obligé de faire ça car il y a des formations mais on ne sait pas ce qu'il y a derrière» (HTMListe «star»).

Enfin, tant en raison de la pénurie de maind'œuvre qualifiée que de la faible lisibilité des compétences, les aspects techniques sont parfois relégués au second rang et les critères de recrutement reposent souvent sur l'adéquation de la personnalité des individus aux valeurs affichées comme étant importantes par l'entreprise et sur leurs expériences professionnelles antérieures dans des secteurs d'activité autres que le web (MARTY, 2002). Ainsi, nombreux sont les babyloniens ayant pris part à des projets professionnels requérant sens de l'initiative et autonomie: «Vu que j'ai monté une boîte avec un pote, j'étais dans l'esprit Babylone» (communication interne). Cette recherche d'individus autonomes s'explique en outre par le fait que la forte croissance de Babylone ne permet pas aux ressources humaines d'encadrer les nouveaux venus qui doivent donc s'intégrer par eux-mêmes: "L'intégration est très dure. On te dit: "bonjour, bienvenu". Et hop, on te lâche dans la nature. On est un peu paumés. Il y a tellement de nouveaux qu'il n'y a pas de stabilité. Au bout de deux trois mois, on nous prend pour un ancien » (concepteur informatique). À tel point que Babylone compte dans ses rangs de nombreux «autodidactes du web» ayant fait la preuve de leur capacité à évoluer de façon autonome dans un cadre organisationnel lâche voire inexistant: "Avant, j'étais musicien. Mon père composant, il avait un ordinateur. J'ai appris comme ça» (HTMListe). À cet égard, ces constats tendent à corroborer l'hypothèse émise par FONDEUR et SAUViat (2002) selon laquelle une évolution rapide de la technologie dans un secteur rend impossible l'existence de qualifications standardisées et confère dès lors une importance capitale à une compétence particulière: la capacité d'adaptation.

\section{Comment limiter le taux de rotation du personnel?}

Pour compenser la pénurie de main-d'œuvre compétente, nombre de start-up ont cherché à fidéliser leurs employés en élaborant des politiques de ressources humaines susceptibles de les dissuader de se laisser séduire par les offres financières de la concurrence. De telles pratiques, similaires à celles observées dans les cabinets de conseil (BENAMOUZIG, 1994), étaient en effet particulièrement fréquentes à cette époque.
Chez Babylone Connection, l'argument le plus visible de cette politique a résidé dans l'octroi de bons de souscription d'actions (BSA) promettant des espoirs de gain importants aux salariés disposés à rester assez longtemps dans l'entreprise pour pouvoir légalement les exercer (cinq ans). En outre, les BSA confèrent aux individus une double casquette de salariés actionnaires (DESBRIĖRES, 1997) et sont pour cette raison supposés susciter chez eux un surcroît de motivation.

La stratégie salariale de l'entreprise est elle aussi orientée vers la fidélisation des employés: si les salaires d'embauche sont légèrement inférieurs à ceux du marché, les augmentations sont rapides et substantielles. En ce sens, cette stratégie peut être rapprochée de l'un des modèles de LAZEAR (1993), dans lequel les salaires sont déconnectés de la productivité des salariés sur le court terme afin de les inciter à ne pas quitter l'entreprise (9).

Parallèlement, la direction de Babylone Connection s'est appuyée sur la qualité de l'ambiance de travail pour prévenir d'éventuels départs en soutenant l'idée d'une conception nouvelle de la vie en entreprise. Cette perspective exerçait un attrait certain sur les jeunes employés de la startup , beaucoup d'entre eux retrouvant dans cet environnement professionnel certains repères du monde étudiant qu'ils venaient de quitter. On observe ainsi dans l'entreprise nombre des clichés concernant la culture du web évoqués avec humour par ICHBIAH (1998).

Cette ambiance est tout d'abord caractérisée par la grande liberté laissée aux babyloniens en matière de «look» (tatouages, piercings, excentricités vestimentaires), d'horaires ou de comportements: «Tes horaires, tout le monde s'en fout: tu peux arriver à 9 heures ou 11 heures, partir à 19 heures ou 23 heures, jouer aux jeux vidéos, bosser avec un casque, venir habillé comme tu veux. L'essentiel, c'est que tu aies fait ton boulot. À partir de 20 heures, les locaux sont «fumeur» et tu peux rouler des joints» (chef de projet). De même, les échanges interpersonnels relèvent plus de la camaraderie que de la relation professionnelle classique (tutoiement généralisé, usage fréquent de pseudonymes, etc.).

Pour beaucoup d'individus, notamment les jeunes originaires de province et ayant un réseau relationnel peu développé à Paris, Babylone représente une seconde maison: "On travaille en s'amusant. C'est une boîte top, pas comme les autres. Certains viennent même le week-end mais pour jouer»" (graphiste). Ce fort sentiment d'appartenance s'explique notamment par les efforts de la direction qui a mis diverses infrastructures à la disposition des

(9) À ce «détail» près que l'échelle de temps envisagée par LAZEAR est longue alors qu'elle n'est que de quelques années voire mois chez Babylone Connection. 
membres du personnel (machine à laver, cuisine et sauna) et organise des fêtes tous les vendredis soir au Babylone Café à l'occasion desquelles «le boss paie à boire à tout le monde». Mais il puise également ses origines dans les multiples divertissements disponibles dans l'entreprise. Au premier rang de ces divertissements, on trouve les jeux en réseau (Quake, Ever Quest), qui semblent constituer un véritable «principe de socialisation professionnelle» (LALlement, 2003). Mais on y inclura également les «mailing lists» qui jouent un rôle crucial dans la cohésion de la communauté babylonienne et constituent une forme de la «communication numérique $(10)$ » caractéristique de la «cyberculture» (LÉvy, 1997). En effet, si certaines ont un contenu purement professionnel (informations par métier ou administratives), d'autres ont un contenu plus ludique: URL de sites, blagues, vie politique, activités sportives, images «porno» et «gore». En ce sens, leur fonction chez Babylone semble proche de celle des forums de discussion étudiés chez France Télécom par Beaudoin, Cardon et Mallard (2001). $\mathrm{Au}$ passage, ces derniers éléments ont motivé des critiques acerbes à l'égard des start-up. Jérémie LEFEBVRE (11) (2000) (12) notamment, compare cet univers à celui de l'île aux Enfants (13) dans la mesure où la culture communautaire infantilise selon lui les individus afin de les rendre dociles.

Enfin, la culture de Babylone Connection est considérée par le personnel comme un motif essentiel de fidélité à l'entreprise. Elle s'inscrit en effet en rupture avec la culture classique des grandes entreprises bureaucratiques pour promouvoir des normes de fonctionnement aux accents libertaires, respectueuses de la culture originelle du web.

La première norme réside dans le refus de l'idée de contrôle. Ce rejet se manifeste d'abord par l'absence de contrôle des comportements : la consommation de cannabis et la circulation d'images pornographiques sont monnaie courante. Il se traduit ensuite par le refus d'un contrôle formel dans le travail. À titre d'exemple, le directeur financier préfère parler de «comptabilité analytique» que de «contrôle de gestion» lorsqu'il décrit son travail et les web timesheets (14) restent à ce jour inutilisables car mal

(10) Moyens de communication transportant l'information par paquets sous la forme de séquences de 0 et de 1 . Il s'agit ici de la communication via Internet mais cette expression englobe aussi la télévision numérique, etc.

(11) Cet ex-employé de l'éditeur de jeux Ubi Soft a été le premier à tenter de créer une représentation syndicale dans une entreprise du secteur des NTIC en lançant en 1998 le site contestataire Ubi Free visant à dénoncer la gestion des ressources humaines d'Ubi Soft (http://membres.lycos.fr/ubifree/).

(12) Cf. WASER, A.-M. (2001) pour un compte rendu de lecture.

(13) Emission télévisée de divertissement diffusée entre 1974 et 1982 et devenue très populaire grâce à la marionnette de Casimir.

(14) Fiches de gestion du temps destinées à facturer la prestation au client. remplies. La politique de la direction consiste donc à responsabiliser les babyloniens et pratiquement tout est permis tant que le travail est fait: «Il faut un temps pour s'adapter à cette structure où on n'oblige à rien. On recrute surtout des gens autonomes, à même de s'intégrer dans une structure souple. Si quelqu'un n'est pas là une journée, personne ne s'inquiète» (manager de studio).

Deuxièmement, les individus de la communauté sont considérés comme égaux et l'idée de hiérarchie est rejetée. Cette valeur a un impact direct sur les relations de travail dans la mesure où l'autorité n'est pas une ressource acceptable pour contraindre un individu à réaliser une tâche: "Il ne faut pas jouer au chef, surtout avec le HTMListe, même si tu es son chef. Sinon, il va dire que tu le prends de haut. La relation doit être d'égal à égal même s'il y a une différence de salaire, de statut. Dans chef de projet, il y a chef et ça ne plaît pas » (chef de projet).

La dernière norme est le refus affiché de l'individualisme et du carriérisme. Les babyloniens disent exercer leur métier par passion et non dans le but de s'assurer une carrière fulgurante: «J'ai des propositions de job à $800 \mathrm{KF}$. Mais l'argent n'est pas un but en soi pour moi. Je me plais bien ici. Il n'y a pas beaucoup de place pour l'évolution en terme de titre et je ne veux pas prendre la place de quelqu'un»" (concepteur expert).

Finalement, les pratiques originales développées par l'entreprise ainsi que la revendication d'une culture de type communautaire aux accents «libertaires » peuvent être appréhendées comme autant de tentatives de s'adapter aux contraintes du marché du travail. Mais loin de se limiter au recrutement et à l'ambiance dans l'entreprise, nous allons voir que les spécificités de ce marché du travail pèsent également sur le fonctionnement interne de l'entreprise.

\section{Des modes de régulation interne répondant aux spécificités du marché du travail}

Nous avons vu que Babylone Connection, comme beaucoup de start-up à cette période, a recruté de nombreux individus dont le niveau de compétence était parfois incertain. Nous allons maintenant voir comment l'organisation du travail et la politique de gestion des hommes en interne permettent de faire face à cette incertitude afin de ne pas mettre l'entreprise en péril.

\section{Le travail en binômes: un processus autonome d'identification et de contrôle des compétences}

Le premier enjeu du processus interne d'organisation du travail consiste à compenser les imperfections du marché du travail en levant le voile d'ignorance dissimulant les niveaux individuels de compétence à l'entrée (problématique de la sélection adverse). Dans le contexte culturel de l'entre- 
prise (refus du contrôle), c'est le travail en binôme qui permet de faire face à ce premier enjeu.

On observe d'abord la formation de binômes composés d'un professionnel et de son «supérieur hiérarchique» direct: développeur informatique/ concepteur informatique et graphiste/directeur artistique. Les deux individus d'un binôme ayant la même formation et les mêmes objectifs, les relations sont en général bonnes. Mais ces binômes n'en demeurent pas moins des lieux de révélation des niveaux individuels de compétence: "[Concepteurs $\mathrm{X}$ et $\mathrm{Y}]$ ont des façons différentes de gérer les gens: $X$ a de l'expérience, c'est une grande valeur ajoutée, alors que $\mathrm{Y}$ est moins technique et m'a aidée en faisant le découpage des images» (développeur informatique).

Parallèlement, on notera l'existence de binômes composés de deux professionnels et formant une chaîne: graphiste/HTMListe puis HTMListe/développeur informatique. Ainsi, chacun doit avancer le travail de son prédécesseur tout en anticipant les contraintes de son successeur. Comme dans les binômes précédents, cette forme d'organisation du travail en chaîne permet à chaque individu d'évaluer le travail de son prédécesseur. Ce phénomène aboutit à une mise au jour de fait du niveau de compétence de chacun: "On avait des modifications à faire sur un site. Le chef de projet a contacté un HTMListe. Mais cette personne a fait du travail pourri et j'ai dû passer derrière: c'est un problème que j'ai eu plusieurs fois chez Babylone» (développeur informatique).

Le travail en binôme constitue ainsi un excellent exemple des «arrangements informels» observés par GIRARD et STARK (2002) dans une web agency de la Silicon Alley new-yorkaise. Ces arrangements, destinés selon eux à simplifier le travail de chacun afin de rendre possible la production d'un site web, constituent un trait dominant d'un nouvel idéaltype d'organisation situé entre la hiérarchie et le marché: "l'hétérarchie». Mais plus pragmatiquement, le travail en binôme s'avère être un moyen de surveillance et de contrôle en ce sens qu'il rend possible un processus émergent d'évaluation de la qualité du travail individuel et une canalisation des comportements non conformes aux attentes de l'entreprise. Notamment, les binômes de professionnels donnent naissance à une forme moins visible mais non moins réelle de contrôle: le contrôle par les pairs (peers pressure). On retrouve d'ailleurs dans ce mode d'organisation une forme classique du concept de «contrôle croisé» développé par REYNAUd (1989).

Finalement, l'analyse de l'organisation du travail rend illusoire l'idée d'une absence de contrôle chez Babylone. Si le contrôle officiel et personnalisé est quasi inexistant, ce sont en fait des mécanismes de contrôle social émergents, moins visibles, moins habituels et souvent non hiérarchiques qui permet- tent de révéler les niveaux individuels de performance. Nous allons maintenant voir comment cette information est diffusée et traitée afin d'assurer le bon fonctionnement du système.

\section{Un système de gestion des carrières peu formalisé: effets de réputation et récompenses implicites}

Le second enjeu majeur consiste à faire remonter et à traiter les informations précédemment obtenues sur les niveaux individuels de compétence sans heurter une culture d'entreprise prônant le refus du carriérisme et l'égalité entre les individus. Pour ce faire, effets de réputation et récompenses implicites doublent efficacement un système officiel de gestion des ressources humaines fondé sur des évaluations formelles et des récompenses classiques.

À l'issue d'un projet, la règle veut que l'équipe soit évaluée par le chef de projet mais également par le manager de studio. Toutefois, cette règle ne s'applique dans les faits que de manière partielle et ne suffit pas à rendre compte des inégalités de compétences. L'évaluation effectuée par le manager de studio n'a que peu de valeur car celui-ci ne suit pas le travail des employés pendant les projets et celle réalisée par le chef de projet reste souvent informelle (grilles prévues non remplies) voire inexistante. Ainsi, de manière générale, les évaluations prévues et formelles n'ont qu'un intérêt limité et pas de répercussions concrètes: «Une évaluation? Je ne sais pas... Ça serait plutôt le chef de projet avec l'aide de mon manager de studio. L'évaluation est informelle: je m'auto-évalue, il m'évalue et on compare. Mon dernier entretien a eu lieu il y a un an et n'a eu aucun impact. C'était du vent» (HTMListe).

Dans ces conditions, l'opinion portée sur les individus semble faiblement liée aux évaluations et repose plutôt sur l'existence de forts effets de réputation. Le personnel de l'entreprise est classé de manière informelle en catégories liées au niveau de compétence: les «bons», «cadors», «top guns» s'opposent ainsi aux «mauvais», «incompétents». À tel point que chaque individu semble avoir une cote au sein de l'entreprise attestant de sa compétence: «Il y a un mec qui porte la chtouille: il transforme en merde tout ce qu'il touche. Et puis il y a [X] qui a des doigts de fée. T'as les bons graphistes et les mauvais. Il y a dix nuisibles qui tirent tout vers le bas et il y a vingt personnes très compétentes » (chef de projet). Cette mise en concurrence des employés est identifiée par BERREBI-HofFMANN (2002) comme un mécanisme de contrôle fréquemment rencontré dans les start-up de même que dans les entreprises informatiques.

Ainsi, les effets de réputation ont une telle ampleur chez Babylone Connection qu'ils se substituent dans les faits au système officiel des évaluations traditionnelles pour devenir «l'indicateur prégnant» (BOUSSARD, 2001) sur lequel repose l'ordre social 
dans l'entreprise. Le seul mécanisme d'évaluation efficient est l'opinion des pairs, fondée sur les informations obtenues lors du travail en binômes puis diffusées par le bouche à oreille. Ce phénomène de diffusion des réputations au sein de l'entreprise relève très exactement de «l'effet-réseau » considéré par Courpasson (2000) dans son étude sur le management par les compétences comme une source de domination: les individus n'ont plus aucun contrôle sur la manière dont ils sont perçus alors même qu'ils sont en théorie censés détenir le pouvoir de construire seuls leurs propres compétences.

Parallèlement, la gestion des hommes chez Babylone Connection comprend un système de «récompenses» destiné à inciter les individus à travailler au maximum de leur productivité.

Tout d'abord, l'entreprise a développé un système classique de récompenses financières et statutaires. Les individus méritants peuvent se voir accorder une prime de fin de projet, une augmentation et/ou une promotion, parfois même sans avoir à la demander, sur décision du chef de projet, du manager de studio ou de la direction. Les salaires d'embauche étant relativement bas, la direction possède une marge de manœuvre importante en termes de politique salariale: «Depuis mon arrivée, j’ai été augmentée une fois sans demander. [Direction] est venu me voir après un mois et m'a dit que j'étais augmentée de $2000 \mathrm{FF}$ » (chef de projet). De même, une évolution statutaire est possible par le biais d'un changement de métier (intégration au sein du studio valorisé des «concepteurs/experts»(15)). Ces promotions demeurent cependant rares en raison de la structure hiérarchique plate de l'entreprise et l'essentiel des promotions correspond au passage très symbolique de «junior» à « confirmé» puis à «senior».

Toutefois, ici encore, ce système officiel de promotion est doublé d'un volet informel et implicite qui se matérialise par l'importance économique des projets dans lesquels les individus sont impliqués. À défaut de leur octroyer une promotion statutaire, ce processus permet de valoriser les meilleurs éléments en les affectant sur des projets de plus en plus importants, tout en garantissant la réussite des projets prioritaires de l'entreprise (gros budgets, enjeux d'image, perspectives de collaborations futures avec le client...). Dès lors, il apparaît que la procédure de staffing (16) constitue la clé de voûte de l'organisation du travail chez Babylone Connection en ce sens qu'elle permet de concilier récompenses implicites destinées à motiver les salariés et intérêts de l'entreprise.

(15) Ce studio récent rassemble six personnes expérimentées supposées assurer un soutien technique en interne et représenter l'expertise de Babylone auprès des clients.

(16) Terme anglais désignant le processus d'affectation des individus sur les projets.

\section{Le staffing: deux poids, deux mesures selon l'importance du projet...}

La procédure de staffing à laquelle donne lieu la phase de production concerne toutes les catégories de professionnels. Elle constitue un enjeu majeur pour les chefs de projet dont l'objectif est d'obtenir des individus travaillant vite et bien afin de respecter les délais et le budget fixés pour un projet. Toutefois, leurs aspirations sont soumises au bon vouloir des managers de studios multimédia et informatique, responsables du staffing, dont le rôle consiste notamment à éviter l'exclusion et la marginalisation des mauvais éléments alors que la start-up est en phase de croissance. Pour ce faire, les managers de studios identifient ces éléments faibles, leur proposent des formations ponctuelles, et surtout les affectent sur des projets en dépit des réticences des chefs de projet.

Cependant, cette procédure officielle donne souvent lieu à des ajustements officieux, les chefs de projet négociant pour obtenir des managers de studio un bon staffing. Et cette négociation peut s'avérer payante: "Le manager de studio connaît les gens: si je demande un top gun, il me le trouve. Mais il ne faut pas abuser» (chef de projet). Ce «contournement» de la procédure officielle de staffing peut avoir divers fondements: assurer la réussite d'un projet prioritaire, prévenir les conflits au sein d'une équipe, respecter le principe d'équité entre chefs de projet («tournante» des mauvais éléments). Mais il semble en fait que la trajectoire du chef de projet (expérience, crédibilité) et surtout l'importance du projet (les deux étant toutefois liés) soient des arguments majeurs: "S'il y a conflit sur le staffing, ça dépend du chef de projet et du projet. Il y a des chefs de projet à qui on ne refuse rien. Ceux qui ont une grande expérience, on leur fait confiance. Si ton projet est moins important, tu te fais piquer les ressources ou t'as les gens moins bons» (chef de projet).

En revanche, la marge de négociation des professionnels eux-mêmes est quasi-nulle dans la mesure où le staffing fait partie du système de récompense officieux. Tout au plus, ils peuvent émettre des préférences thématiques (secteur ou technologie) ou exprimer leur insatisfaction en modérant leur ardeur au travail: "Ils font la gueule mais ne peuvent pas faire plus» (chef de projet).

De manière générale, on retrouve donc durant cette phase de staffing des professionnels les arrangements ainsi que les enchevêtrements de relations marchandes et hiérarchiques décrits par GIRARD et Stark (2002). À tel point que deux tendances lourdes se dégagent selon l'importance du projet considéré: 
- Les projets prioritaires :

la dynamique des «réseaux d'excellence»

Sur les projets prioritaires, les processus officieux de négociation chefs de projet/managers de studio évoqués précédemment jouent à plein. En effet, ces projets sont en général confiés à des chefs de projet expérimentés disposant d'un «capital crédibilité » et d'un pouvoir de négociation fort face aux managers de studio en termes de staffing. Aussi, les équipes projet regroupent majoritairement de très bons éléments: à la période de l'étude, les deux «stars du HTML» étaient staffés sur les deux projets prioritaires de l'entreprise, et l'ancien chef de projet de l'un de ces projets venait d'être promu directeur des opérations internationales, signe de la confiance que lui accordait la direction. Ainsi, une logique d'appariement (Menger, 1994) par niveau de compétence préside à la composition des équipes travaillant sur les projets prioritaires. Cette logique de «réseaux d'excellence» répond à des impératifs économiques: elle optimise les chances de réussite des projets capitaux pour l'entreprise.

- Les autres projets :

l'application de la règle officielle

Les projets non prioritaires sont soumis à la «règle de droit commun »: les chefs de projet ne choisissent pas les professionnels et sont dépendants des managers de studio qui maîtrisent les incertitudes du staffing. Certes, des négociations existent ici encore, mais qui jouent à la marge et n'aboutissent qu'à des ajustements ponctuels (éviter les conflits par exemple): ni l'importance des projets ni la légitimité des chefs de projet ne contraignent les managers de studio à transgresser de manière flagrante la règle. Ainsi, la majorité de l'effectif de Babylone, à l'exception des meilleurs éléments (et de quelques autres affectés aléatoirement sur les projets prioritaires), est soumise à la règle officielle. Cette règle garantit notamment le principe d'équité : les chefs de projet doivent accepter de travailler avec tous les professionnels, y compris les «mauvais», et réciproquement. Dans ces conditions, un objectif fondamental est atteint: les moins bons éléments ne sont pas exclus de l'organisation babylonienne. Ils peuvent alors progresser au contact des autres éléments ou en bénéficiant de périodes sans staffing: «J'ai proposé de dire aux gens moins bons pourquoi on rechigne à travailler avec eux. Bien sûr, ils se retranchent mais je leur fais remarquer qu'ils ne maîtrisent pas tel logiciel [...]. Je leur propose alors de changer les choses et je leur donne quinze jours sans staffing pour apprendre tel truc [...]» (manager de studio).

Finalement, les observations formulées convergent vers une même conclusion: le modèle organisationnel babylonien est caractérisé par une sélectivité indéniable permettant de compenser les imperfections d'un recrutement rendu complexe par le phénomène de sélection adverse. En effet, le fonctionnement interne de la start-up permet de lever le voile sur les niveaux individuels de compétence, de les diffuser par le système des réputations et enfin, d'organiser le travail de façon à prendre en compte ces inégalités. Cette sélectivité est toutefois rarement ouverte et personnalisée et ne donne pas lieu à des phénomènes d'exclusion: les éléments moins compétents sont généralement formés plutôt que licenciés. Globalement, ces procédés permettent donc à Babylone Connection de faire face à la double problématique de rareté des ressources humaines et d'incertitude initiale sur leur qualité. Ils s'avèrent donc adaptés à l'environnement instable de l'entreprise(17).

À cet égard, on s'étonnera de trouver à l'œuvre dans une organisation capitaliste moderne un système de récompense de type «bureaucratique» fondé avant tout sur l'intérêt et l'importance du travail qu'un individu se voit confier. À la différence que cet état de fait découle de la proéminence des règles impersonnelles dans les bureaucraties alors qu'il est lié pour Babylone Connection à une structure particulière du marché du travail qui rend impossible la motivation des individus par la perspective d'une évolution de carrière rapide ou d'un licenciement. Dans ces conditions, le cas Babylone Connection propose un paradoxe inattendu: alors que cette start-up constitue une figure emblématique du capitalisme actuel, sa marge de manœuvre en matière de gestion des ressources humaines est singulièrement entravée par la structure de son marché du travail.

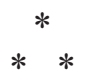

Dans cet article, nous avons montré qu'une pénurie et une absence de «signal» des compétences sur le marché du travail impliquaient des ajustements organisationnels significatifs de la part des organisations du secteur d'activité concerné. Pour ce faire, nous avons évoqué l'émergence récente d'une innovation technologique majeure, l'Internet, qui a conféré une valeur précieuse à des compétences nouvelles, rares et difficilement identifiables. Nous avons illustré notre propos en donnant à voir les stratégies de recrutement et d'organisation interne du travail développées par la web agency Babylone Connection en réaction à cette conjoncture exceptionnelle du marché du travail. À la lueur des résultats obtenus, trois éléments principaux de conclusion seront proposés.

(17) La structure du marché du travail du secteur de l'Internet s'étant depuis inversée (abondance de compétences clairement identifiables), les mêmes appariements sélectifs déboucheraient probablement aujourd'hui sur une exclusion plus directe des individus moins compétents (licenciement pur et simple), comme décrit par Menger (1994) dans le monde artistique (inactivité des artistes les moins réputés). 
En premier lieu, on se demandera si les résultats présentés dans ce papier peuvent être étendus à d'autres web agencies et start-up high-tech en général. De fait, des similarités existent entre les observations portant sur le fonctionnement de Babylone Connection et les cas comparables rapportés par la littérature: recrutement fondé sur l'adéquation aux valeurs de la start-up et sur la personnalité des candidats plus que sur leurs compétences (MARTY, 2002), fonctionnement reposant sur des arrangements et sur un enchevêtrement de relations marchandes et hiérarchiques dans les web agencies (GIRARD, STARK, 2002), contrôle exercé dans les start-up par la mise en concurrence des employés (Berrebi-Hoffmann, 2002), etc. De même, le fonctionnement de Babylone Connection est étonnamment proche de l'un des cinq modèles de fonctionnement des entreprises high-tech répertoriés par BARON, Burton, Hannan (1999): celui du commitment, caractérisé par un fort attachement des employés à l'entreprise «love», une sélection à l'entrée ciblant des profils adaptés à la culture de l'entreprise (cultural fit), et l'existence d'un contrôle exercé par la culture et les pairs (peer/cultural).

En admettant qu'elle soit validée, cette généralisation porte en outre en germe une question intéressante : la structure du marché du travail de l'Internet au milieu des années 1990, dont l'impact organisationnel a été décrit, peut-elle contribuer à expliquer l'originalité du fonctionnement des start-up, au même titre que la culture spécifique du web généralement mise en avant? Force est de constater que le choix d'une majorité de start-up de renoncer à partir de 2001 à leurs excentricités pour devenir des «entreprises comme les autres», s'il participe d'une quête de légitimité liée à la crise de la net économie, coïncide curieusement aussi avec le retournement du marché du travail (abondance de main-d'œuvre, émergence de formations reconnues, etc.).

En second lieu, les résultats de cet article conduisent à se demander si une pénurie de main-d'œuvre ou un flou sur les compétences ont un impact similaire sur une jeune start-up et sur une entreprise plus établie. AutIER et PICQ (2002) ont apporté des éléments de réponse à cette interrogation: ils ont montré que, contrairement aux prédictions des modèles classiques fondant l'avantage comparatif d'une entreprise sur ses ressources rares et spécifiques, les entreprises du secteur du jeu vidéo tendent en se développant à se séparer de leurs ressources humaines irremplaçables au profit de ressources génériques, moins rares et plus aisées à repérer. Leurs résultats semblent donc inviter à considérer le fonctionnement de Babylone Connection et d'autres start-up comme lié à un effet de taille et d'âge plus que comme une réponse à la conjoncture du marché du travail. Pourtant, à y regarder de plus près, leurs conclusions sont doublement problématiques. Tout d'abord, Vernardet (1999) a montré dans son étude sur l'industrie du jeu vidéo que si les bons créatifs, ressource rare et précieuse, quittent effectivement les studios lorsque ceux-ci atteignent une taille critique les amenant à privilégier les impératifs économiques aux considérations artistiques, ils y reviennent par la suite dans la mesure où les studios de grande taille ou rattachés à des éditeurs leur assurent des revenus stables et conséquents. D'autre part, la pertinence d'une ressource ne doit pas être décontextualisée et on peut opposer à F. Autier et T. PICQ que la ressource humaine stratégique change peut être lorsqu'une start-up se développe: incarnée d'abord par le créatif génial capable d'imaginer un jeu innovant pour lancer l'entreprise, pourquoi ne serait-elle pas représentée ensuite par l'habile stratège qui saura optimiser les ventes du même jeu?

En troisième et dernier lieu, on peut se demander si d'autres organisations n'auraient pas pu apporter au milieu des années 1990 des réponses organisationnelles différentes de celle de Babylone Connection à la conjoncture du marché du travail. On aurait par exemple pu s'attendre à observer chez Babylone Connection un fonctionnement de type up or out(18) (O'FlaherTy, Siow, 1995), fréquent dans les entreprises confrontées à des problèmes de sélection adverse lors du recrutement comme les cabinets de conseil (Fondeur, Sauviat, 2002), d'audit ou d'avocats. Or tel n'est pas le cas dans la mesure où ces modèles, bien qu'adaptés aux cas de sélection adverse, ne constituent pas une réponse satisfaisante à une pénurie de main-d'œuvre. Ainsi, chez Babylone Connection, les individus incompétents sont identifiés puis formés plutôt que licenciés : il est en effet plus judicieux de former un individu peu compétent partageant les valeurs de l'entreprise que de lancer une nouvelle procédure de recrutement comportant inéluctablement les mêmes incertitudes.

Trois éléments de conclusion qui constituent autant de nouvelles pistes de recherche à explorer pour mieux comprendre l'impact réel d'un marché du travail tendu sur le fonctionnement des organisations du secteur.
(18) Selon la règle du up or out («être promu ou partir»), les collaborateurs jugés capables par leur hiérarchie d'occuper un poste plus important sont promus, les autres sont invités à quitter l'entreprise. 


\section{Bibliographie}

Akerlof G.A. (1970), “The Market for 'Lemons': Quality Uncertainty and the Market Mecanism", The Quaterly Journal of Economics, 84 (3), pp. 488 - 500.

Autier F., PicQ T. (2002), «Gestion stratégique des ressources humaines dans le secteur du jeu vidéo: une situation paradoxale », Actes du XIIIe congrès de l'AGRH, Nantes.

Baron J.N., Burton M.D., Hannan M.T. (1999), "Building the iron cage: determinants of managerial intensity in the early years of organizations", American Sociological Review, 64, pp. 527-547.

Beaudoin V., Cardon D., Mallard A. (2001), «De clic en clic - Créativité et rationalisation dans les usages des intranets d'entreprise », Sociologie du Travail, 43 (3), pp. 309-326.

Benamouzig D. (1994), «Configurations de pouvoir et socialisation individuelle dans un cabinet de conseil», Sociologie du Travail, 36 (3), pp. 293-314.

Berrebi-Hofmann I. (2002), «Nouvelleéconomie, nouveaux pouvoirs?», Sciences Humaines, 125, pp. 32-35.

Boltanski L., Chiapello E. (1999), Le nouvel esprit du capitalisme, Gallimard, nrf Essais, Paris.

Boussard V. (2001), «Quand les règles s'incarnent L'exemple des indicateurs prégnants», Sociologie $d u$ Travail, 43 (4), pp. 533-551.

Bureau M.-C., Marchal E. (eds) (2005), Au risque de l'évaluation. Salariés et candidats à l'emploi soumis aux aléas du jugement, Presses universitaires du Septentrion, Villeneuve-d'Ascq.

Courpasson D. (2000), L'Action contrainte. Organisation libérales et domination, PUF, Paris.

Courpasson D., Livian Y.-F. (1991), «Le développement récent de la notion de compétence. Glissement sémantique ou idéologie?», Revue de Gestion des Ressources Humaines, 1, pp. 3-9.

Crozier M., Friedberg E. (1977), L'Acteur et le système, Seuil, Paris.

Desbrières P. (1997), «La participation financière des salariés et ses incidences sur la performance et l'organisation interne de l'entreprise». In: Charreaux G. (ed.), Le gouvernement des entreprises, Economica, Paris.

Dugué E. (1994), «La gestion des compétences: les savoirs dévalués, le pouvoir occulté», Sociologie $d u$ travail, 36 (3), pp. 273-291.

Durand J.-P. (2000), «Les enjeux de la logique compétence», Gérer et Comprendre, 62, pp. 16-24.

Eymard-Duvernay F., Marchal E. (1997), Façons de recruter, Paris, Métailié.

Eymard-Duvernay F., Marchal E. (2000), «Qui calcule trop finit par déraisonner: les experts du marché du travail», Sociologie du travail, 42, pp. 411-432.

Fondeur Y., Sauviat C. (2002), Normes d'emploi et marché du travail dans les métiers liés aux technologies de l'information, document d'études Dares, 63.

GIRARD M., STARK D. (2002), "Distributing Intelligence and Organizing Diversity in New Media Projects", Environment and Planning A, 34 (11).

Granovetter M.S. (1974), Getting a job; a study of contacts and careers, University of Chicago Press.
IChBiah D. (1998), Cyberculture, Anne Carrière, Paris. Kait, C., Weiss, S. (2001), Digital Hustlers: Living Large and Falling Hard in Silicon Alley, Harper Collins, New York.

Kerr C. (1954), "The Balkanisation of Labor Markets". In Bakke E.W. (ed.), Labor Market Mobility and Economic Opportunity, MIT Press, Cambridge, pp. 92-110.

Lallement M. (2003), Temps, travail et modes de vie, PUF, Paris.

Lazear E.P. (1993), Personnel Economics. "Chap. 4: Work-Life Incentives Schemes" (p 39 - 46), Editions Wicksell Lectures, Stokholm.

Le Boterf G. (2000), Construire les compétences individuelles et collectives, Éditions d'Organisation, Paris (3e édition).

Lefebvre J. (2000), La Société de consolation, Sens et Tonka, Paris.

LEFRESNE F. (2002), «Vers un renouvellement de l'analyse segmentationniste», Économie et société - série Socioéconomie du travail, 22, pp. 1241-1268.

Levy P. (1997), Cyberculture - rapport au Conseil de l'Europe, Odile Jacob, Paris.

Lichtenberger Y., Paradeise C. (2001), «Compétences, compétences », Sociologie du travail, 43 (1), p. 33-48.

MarTy O. (2002), «La vie de start-up. (S')investir dans les entreprises innovantes», Gérer et Comprendre, 67, pp.4-15.

Menger P.-M. (1994), Appariement, risque et capital humain: l'emploi et la carrière dans les professions artistiques. L'art de la recherche. Essais en l'honneur de Raymonde Moulin, La Documentation française, Paris, pp. 219-238.

O’Flaherty B., Siow A. (1992), “On the job screening, up or out rules, and firm growth", Canadian Journal of Economics, 25 (2), pp. 346-368.

OIRY E., D’IrIBARNEA. (2001), «La Notion de compétence : continuités et changements par rapport à la notion de qualification », Sociologie du travail, 43 (1), pp. 49-66.

Reynaud J.-D. (1997), Les Règles du jeu, Armand Colin/ Masson (1re édition: 1989), Paris.

Riou N. (2001), Comment j'ai foiré ma start-up, Éditions d'Organisation, Paris.

Spence M.A. (1973), “Job Market Signaling”, Quaterly Journal of Economics, 87, pp. 355-379.

Vernardet J. (1999), Les Nouveaux maîtres du jeu. Une étude de la filière logiciels de loisirs, mémoire présenté pour le DEA de sociologie de l'IEP de Paris.

WASER A.-M. (2001), "Vous êtes libres?», Gérer et Comprendre, 64, pp. 54-55.

ZARIFIAN P. (1988), «Le modèle de la compétence». In Stankiewick, F. (dir.), Les Stratégies d'entreprises face aux ressources humaines: l'après-taylorisme, Economica, Paris.

ZARIFIAN P. (2000), «Sur la question de la compétence, réponse à Jean-Pierre Durand», Gérer et Comprendre, 62 , pp. 25-28.

ZARIFIAN P. (2001), Le Modèle de la compétence: trajectoires historiques, enjeux actuels et propositions, Liaisons, Paris. 


\title{
Les catégories d'emploi dans la société japonaise
}

\author{
Kurumi Sugita $(*)$
}

\begin{abstract}
Les praticiens des comparaisons internationales en sciences sociales sont confrontés à la question de la comparabilité de leurs outils d'analyse, et notamment des catégories statistiques ou conceptuelles qu'ils emploient. L'auteure traite ici des catégories d'emploi telles qu'elles sont utilisées dans les enquêtes sur l'emploi et le travail au Japon. Elle part d'un examen minutieux des questionnaires de différentes enquêtes sur l'emploi pour observer les divers usages qui sont faits de ces catégories, dans une double perspective, historique (examen des variations de sens au cours du temps) et transversale (variations d'une enquête à l'autre). L'usage de ces catégories nécessite beaucoup de précautions, et doit tenir compte des relations existant entre elles, et les resituer dans leur contexte historique. L'auteure met en évidence le flou qui entoure désormais certaines catégories du fait de l'effacement progressif de leur signification lié à la sédimentation des enquêtes. Elle en souligne le caractère équivoque, du fait en particulier du non-recouvrement entre des stratifications de significations successives, ce qui a rendu flou le contour de certaines d'entre elles. L'article donne plusieurs interprétations possibles de leur caractère équivoque. Par exemple, le fait que la dénomination usuelle et quotidienne des différentes formes d'emploi soit également utilisée par le système d'observation statistique. Ou le fait encore que le système de classification savant se base sur les pratiques successives des entreprises, témoignant de leur importance dans la société japonaise. En tant que constructions sociales, les catégories du système statistique sont porteuses des évolutions de la société, incorporant au fil du temps des significations suivant des logiques différentes, qui contribuent à les rendre ambiguës, et peuvent prêter à confusion. Un glossaire en annexe aide à démêler ces imbrications de sens.
\end{abstract}

L'objet de cet article est d'analyser les catégories d'emploi telles qu'elles sont utilisées actuellement dans le système statistique japonais, et de rendre compte de la cohérence et des tensions qui existent entre elles. Les catégories d'emploi sont considérées ici comme des constructions sociales interdépendantes, se définissant les unes par rapport aux autres. Ce sont les relations entre elles, et la configuration de l'ensemble de ces catégories qui nous intéresseront. Dire que les catégories d'emploi sont des constructions sociales signifie qu'elles peuvent changer au fil du temps. Je vais donc les examiner dans leur dimension historique, sans toutefois m'engager dans l'étude de leur première mise en place. Le présent article se limitera à l'examen de leur évolution, et tendra à mettre en évidence les ressorts sociaux des définitions produites au fil du temps à la fois par les institutions statistiques, par les entreprises et par les individus eux-mêmes dans les réponses qu'ils font aux enquêtes.

Pour ce faire, je m'appuierai sur l'étude des questionnaires de quelques enquêtes publiques concernant la force de travail. Je n'aborderai pas ici la question de la qualité des réponses, fondées sur l'auto-évaluation, ni la nécessité de les replacer

\footnotetext{
* Chercheuse à l'Institut d'Asie Orientale, ENS LHS Lyon; kurumi.sugita@ens-1sh.fr
}

dans leur contexte. J'analyserai les questionnaires des enquêtes nationales et tout spécialement leur terminologie. J'utiliserai comme corpus de données la traduction officielle en anglais fournie dans les rapports d'enquêtes, car cette traduction est souvent révélatrice de la complexité latente des catégories(1). Je ne traiterai pas ici des catégories juridiques, pourtant étroitement imbriquées avec les catégories statistiques, l'articulation dynamique entre ces deux champs méritant une analyse ultérieure (2). L'article commencera par une esquisse de l'histoire des entreprises et du salariat au Japon, afin de montrer l'importance du rôle social de l'entreprise dans la société japonaise, perceptible dans la part qu'elle a eue dans la construction du système de classification. Cette esquisse est structurée autour des formes d'emploi et de la mobilité professionnelle. Elle examine l'émergence et la stabilisation de la norme dite de «l'emploi à vie», et met en lumière comment ce processus $\mathrm{a}$, simulta-

(1) Cette approche s'inscrit plus généralement dans les travaux anthropologiques sur le système de classification ( $c f$. DougLas, 1986; Douglas, Hill, 1992). Pour la France on peut évoquer A. Desrosières $(1988,2000,2001,2003)$.

(2) De même, le système de production statistique au Japon n'est pas analysé. La question de la place de l'État dans ce système devrait être étudiée pour mieux préciser le rôle que jouent les modalités de l'observation statistique aussi bien dans la mise en place des catégories que dans les politiques publiques. 
nément, fait émerger d'autres catégories d'emploi. La seconde partie de l'article présente les grandes enquêtes japonaises sur l'emploi, conduites par le Statistics Bureau of Ministry of Internal Affairs and communications du Japon. Les catégories d'emploi qu'elles utilisent sont analysées dans la dernière partie de l'article sous l'angle du statut d'emploi et de la forme d'emploi, mettant en évidence un cumul de significations successives à travers l'histoire.

\section{Les entreprises japonaises et le salariat}

$\mathrm{Au}$ Japon, de nombreux éléments pris en charge en France par les pouvoirs publics ont été historiquement dévolus aux entreprises, selon des rapports sociaux structurant les catégories d'emploi en fonction des attributs d'âge ou de genre des individus.

\section{Jusqu'à la fin de la Seconde Guerre mondiale}

Dans la seconde moitié du XIX $\mathrm{X}^{\mathrm{e}}$ siècle, les entreprises d'État jouèrent un rôle essentiel dans l'industrie lourde à cause de l'importance de la production militaire. Cette industrie profita largement des deux guerres sino-japonaise (1894-1895) et russo-japonaise (1904-1905). Par la suite, son développement s'est accompagné d'une modernisation technologique réalisée surtout grâce à la coopération des entreprises occidentales au cours des années 1920.

Dans le secteur des industries légères, le relais de l'État fut pris par des entreprises privées dans les années 1880 et 1890 . Ainsi l'industrie textile s'attacha très tôt à combler son retard sur la technologie occidentale, et les progrès techniques y furent comparativement plus rapides.

Par ailleurs, le développement de l'industrie lourde impliquait une évolution dans la formation et la qualification ouvrière. Dans ce secteur, le système de formation le plus ancien était l'institution des oyakata (maître/père), dont l'origine se trouve dans le milieu artisanal traditionnel. La majeure partie des ouvriers était placée sous le contrôle des oyakata, intermédiaires entre les ouvriers et l'entrepriseemployeur. Il s'agissait d'ouvriers ayant acquis une compétence grâce à leurs contacts avec d'autres ouvriers qualifiés ou avec des ingénieurs étrangers. Certains oyakata contrôlaient des centaines de travailleurs et étaient, de fait, des sous-traitants de l'entreprise. L'entreprise confiait à un oyakata une tâche à accomplir et celui-ci organisait le travail et la rémunération comme il l'entendait (HAZAMA, 1976, 1978; Gordon, 1988; Thomann, 2005).

L'apprentissage était fondé sur l'observation et l'imitation: les apprentis se formaient en regardant faire l'oyakata et les ouvriers plus âgés. Cependant, pour répondre aux besoins de formation plus systé- matiques des ouvriers, le gouvernement mit en place des écoles techniques dès la fin du XIXe siècle, suivi par les grandes entreprises privées. Ce type de formation se répandit progressivement avant la Première Guerre mondiale (Hazama, 1978). Après la guerre, les grandes entreprises se mirent à pratiquer systématiquement la formation interne, qu'elles dispensaient aux jeunes recrutés directement après leur formation scolaire (SumIYA, 1977). Le remplacement d'une qualification artisanale et la disparition de l'institution des oyakata, de pair avec l'introduction de l'Organisation scientifique du travail, marquent pour la première fois l'apparition de relations salariales directes entre les travailleurs et l'entreprise dans le secteur de l'industrie lourde. Par contre, dans l'industrie légère, notamment textile, l'institution des oyakata n'existait pas. La mécanisation qui caractérisait cette industrie favorisait le contrôle direct de la main-d'œuvre par l'entreprise, main-d'œuvre composée essentiellement de jeunes femmes dont la formation se faisait presque exclusivement sur le tas. Il existait des écoles d'entreprise, mais ces établissements enseignaient les arts domestiques féminins.

Après la Première Guerre mondiale se met en place une population ouvrière qui constituera, ultérieurement, le cœur de la norme d'emploi dominante: celle des ouvriers qualifiés de l'industrie lourde, appartenant surtout à de grandes entreprises, ouvriers toute leur vie et sans autres ressources. Par ailleurs, la grande partie des ouvriers non qualifiés effectuait un travail saisonnier durant la saison creuse agricole, ou bien restait fermiers et travaillait temporairement dans les usines (3).

La disparition progressive du système des oyakata s'est accompagnée d'une politique d'entreprise visant à stabiliser les ouvriers qualifiés, très demandés, mais qui changeaient fréquemment d'emploi à la recherche de meilleures conditions de travail. Dès les années 1910, les grandes entreprises ont introduit des politiques d'avantages sociaux appliqués exclusivement aux ouvriers qualifiés, consolidant ainsi la distinction entre ouvriers réguliers qualifiés (jôko ou jôyô koyô) et ouvriers temporaires non ou peu qualifiés. Cette politique ne s'appliquait pas uniformément à tous les ouvriers permanents; elle touchait surtout les plus anciens. Tous ces éléments - politique de formation, établissement d'un statut privilégié, attribution d'avantages sociaux - aboutirent à la constitution d'un marché du travail fermé où la mobilité était essentiellement interne à l'entreprise. Le salaire

(3) Le travail «en alternance» entre l'industrie et la campagne, appelé dekasegi, caractérise une partie de la main-d'œuvre japonaise jusqu'à nos jours (voir ÔKAWA, 1979; WATANABE, HANEDA, 1977). Le terme dekasegi est actuellement employé pour désigner les immigrés issus de la migration japonaise outre-mer, principalement en Amérique du Sud, qui retournent au Japon pour y travailler. 
à l'ancienneté fait partie de cette stratégie de stabilisation de la main-d'œuvre qualifiée.

Les relations salariales qui se structurent ainsi dans la première moitié du $\mathrm{XX}^{\mathrm{e}}$ siècle doivent être replacées dans un contexte historique dans lequel les instances politiques et économiques sont profondément imbriquées avec la structure de parenté. L'État et l'entreprise étaient présentés comme un prolongement de l'organisation familiale. Dans le discours politique et patronal, le modèle de référence était la famille patriarcale dans laquelle la continuité du lignage était un absolu. La piété filiale acquise dans la famille, décrite comme le fondement de la morale et de l'éthique, était en continuité directe avec la loyauté envers l'empereur. L'effort des organisations politiques et économiques pour étendre ces règles familiales à l'organisation du travail est repérable dans les discours patronaux contre la promulgation $\mathrm{du}$ Code du travail, lequel risquait d'introduire, selon eux, un cadre juridique nouveau et externe dans les relations salariales, alors que ces dernières devraient être régies dans le cadre de la parenté.

Pendant la Seconde Guerre mondiale, et surtout vers sa fin, le salaire a progressivement été dissocié du travail effectué, et a acquis un caractère de revenu de survie pour le travailleur et sa famille, dans le cadre de la mobilisation pour la guerre. Ainsi, la famille d'un ouvrier mobilisé continuait à recevoir son salaire pendant son absence, ce salaire étant considéré comme une rétribution du soldat de l'armée impériale. Cette dissociation du salaire et du travail effectif, son association à la survie de la famille auraient préparé la forme du salaire dit «vital», instituée après guerre à la suite d'une forte mobilisation syndicale (MAGOTA, 1978).

\section{La fin de la guerre et la période de haute croissance économique}

Après la guerre, les syndicats mobilisent contre les licenciements, revendiquant la sécurité d'emploi et un salaire minimum basé sur le coût de survie. La partie centrale du salaire, qui a marqué le système ultérieur, était calculée en fonction de l'âge du travailleur, et avait comme objectif d'assurer la vie du salarié et de sa famille. À cette partie s'ajoutaient des éléments prenant en compte la compétence et l'ancienneté (Urabe, ÔMURA, 1983).

Cette forme du salaire est fortement liée à l'institution familiale. À travers la sécurité d'emploi et le revenu du chef de famille masculin, l'entreprise est censée assurer la survie de la famille. Dans ce cadre, la fonction de régulation de l'emploi ainsi que la protection sociale étaient déléguées en partie à l'entreprise. De même, l'institution familiale, intégrée dans cette configuration, fournissait la base de services aux membres de la famille à la place des organismes publics ou d'État : les femmes assuraient ces services, jouant le rôle combiné de travailleuse/ mère/épouse/bru, parfois en se retirant du marché du travail, et souvent en travaillant en tant qu'aide familiale ou à temps partiel.

Pendant la période de forte croissance économique, 1955-1970, les grandes entreprises japonaises évitaient autant que possible les compressions d'effectifs; elles ont ainsi progressivement institué la norme d'emploi dite «emploi à vie», shûshin koyô.

C'est à travers le développement du débat sur la gestion «à la japonaise», dans lequel les médias ont joué un rôle très important, que la pratique de l'emploi à long terme, apparue dans les années 1920 dans le contexte de la modernisation de l'industrie lourde, défendue par les syndicats d'entreprise après la Seconde Guerre mondiale, se voit instituée comme concept clé de la forme d'emploi (Nomura, 1994; Sugita, 1989). Les termes d' «emploi à vie» (shûshin koyô) et "salaire à l'ancienneté» (nenkô chingin) se stabilisent et entrent dans le langage ordinaire au cours des années 1960 et 1970.

\section{La restructuration de l'organisation de la production}

Vers la fin des années 1960 et au début des années 1970, les grandes entreprises se lancent à la fois dans la rationalisation intérieure et dans la réorganisation des sous-traitants. La grande majorité des entreprises japonaises est intégrée dans des chaînes de production chapeautées par une grande entreprise de plus en plus spécialisée dans la conception et la commercialisation. La production est assurée par une cascade de sous-traitants qui sont organisés de manière extrêmement hiérarchisée. Le modèle de référence pour une entreprise japonaise serait de démarrer au bas de l'échelle, puis de grimper en assumant des processus de production de plus en plus difficiles du point de vue technologique ou organisationnel, et en créant ses propres réseaux de sous-traitants. Cette ascension a des conséquences économiques immédiates, en particulier au niveau des prix et des salaires, car le prix de l'heure achetée/ vendue dans les transactions dépend de la place de l'entreprise dans cette hiérarchie. L'ascension de l'entreprise dans l'échelle a une répercussion directe sur le personnel. Du fait du statut plus élevé de son entreprise, le salarié jouira lui-même d'un prestige et d'un salaire plus élevés, et d'une meilleure sécurité de l'emploi. Ainsi, la mobilité est située plutôt au niveau de l'entreprise qu'au niveau de l'individu. La carrière professionnelle d'un individu est étroitement liée à l'histoire de l'entreprise. Par ailleurs, jusqu'à la crise actuelle, l'entreprise japonaise fonctionnait sur la base d'un champ d'action temporel très long. Les participations croisées dans les grandes entreprises, l'engagement à long terme de la banque principale, permettaient aux entreprises d'adopter une logique stratégique à un horizon temporel fort 
éloigné. Dans ce cadre, avec la norme d'emploi à vie, les membres d'une entreprise partageaient intimement un vécu et un avenir collectifs (MAGAUD, Sugita, 1993 ; Sugita, 1997).

\section{La politique de flexibilisation du marché de l'emploi et l'éclatement des bulles financières}

En 1985, une nouvelle loi, the Worker Dispatching Law(4), autorise les entreprises intérimaires dans certains secteurs d'activité; en 1999, un amendement à la loi les autorise dans presque tous les secteurs, sauf dans des cas bien précis. Un dernier amendement, en 2004, a prolongé la durée du contrat d'un an à trois ans sous certaines conditions.

L'éclatement des bulles financières en 1992 et la crise économique qui a suivi ont accéléré l'effritement du système d'emploi, suscitant la crise de la norme de «l'emploi à vie», et menaçant une population autrefois bien protégée, à savoir les salariés réguliers masculins âgés de 35 ans à 54 ans, chefs de famille, travaillant dans des grandes entreprises. L'emploi dit atypique augmente de manière forte depuis les années 1980 et en particulier dans les années 1990, et participe à la déstabilisation de la norme principale d'emploi. La hausse du taux de chômage, en particulier chez les jeunes, et l'instabilité croissante des trajectoires professionnelles modifient profondément les perspectives de vie. Le licenciement et la difficulté de retrouver un emploi chez les hommes ayant connu une longue carrière stable bouleversent la personne et menacent la division sexuelle du travail au sein de la famille (Kase, Sugita, 2006).

\section{Les enquêtes japonaises sur l'emploi}

Les catégories d'emploi au Japon vont être étudiées maintenant à partir des définitions qui en sont données dans les questionnaires des trois enquêtes conduites par le Statistics Bureau of Ministry of Internal Affaires and Communications du Japon. Ce sont les enquêtes de base sur l'emploi au Japon. Conçues et réalisées par le même organisme, elles sont relativement homogènes, ce qui va faciliter le travail de déconstruction des catégories utilisées.

Le questionnaire n'est pas rempli par l'enquêteur dans le cadre d'un entretien en face à face, mais distribué par l'enquêteur qui le récupère ensuite chez les répondants.

\section{Labour Force Survey}

L'équivalent de l'Enquête Emploi, rôdô ryoku chôsa - Labour Force Survey (LFS ci-après) - a été introduit au Japon en 1946 sous la direction des Américains suivant le modèle du Current Population Survey (CPS ci-après) aux États-Unis (IWAI, 1992). La LFS a commencé véritablement en 1947 après un an d'essai. Elle est réalisée auprès d'environ 40000 ménages, soit environ 100000 personnes de 15 ans et plus. Le même échantillon est suivi pendant deux mois. Elle porte sur la dernière semaine de chaque mois (dans le cas du mois de décembre, il s'agit de la semaine du 20 au 26). Cette enquête ainsi que la Special Survey of the LFS (voir ci-dessous), suit la convention du $\mathrm{BIT}$, et porte sur la semaine de référence.

La LFS comprend en sus des questions concernant l'identité, la position au sein du ménage et l'état civil: neuf questions au total pour les personnes actives occupées, deux questions pour les personnes qui n'ont pas travaillé dans la semaine de référence et qui cherchent un emploi, et une question pour les inactifs. La différence dans la taille du questionnaire entre la LFS d'une part et la CPS ou l'Enquête Emploi en France de l'autre est flagrante: la partie du questionnaire de la CPS sur l'emploi comporte environ 200 questions. Le questionnaire de l'Enquête Emploi comporte, pour les personnes ayant une activité professionnelle, grosso modo une soixantaine de questions (nombre variable selon les filtres), et une trentaine de questions pour les personnes s'étant déclarées «chômeur».

Cette enquête de taille restreinte est complétée par la Special Survey of the Labour Force Survey (SSLFS ci-après), conduite une fois par an à peu près sur un échantillon de même taille - éventuellement un peu plus faible. Cette enquête a été réalisée pour la première fois en 1949 en tant qu'enquête complémentaire à la LFS. Elle porte le nom de SSLFS à partir de 1962. Depuis 1999, elle est effectuée deux fois par an. À partir de 2002, elle est intégrée dans la LFS en tant que questionnaire spécial. J'examinerai ici le questionnaire SSLFS aussi bien que le questionnaire spécial de la LFS qui l'a remplacé. Quant au volume, le questionnaire de 1964 comporte, sur l'emploi, cinq questions au plus pour les actifs, et quatre questions pour les inactifs. Le nouveau questionnaire de 2002 comprend treize questions au plus pour les personnes de différents statuts (actif occupé, chômeur, inactif).

\section{Employment Status Survey (Shûgyô kôzô kihon chôsa)}

Cette enquête a démarré en 1956 au rythme d'une enquête tous les trois ans. À partir de 1982, elle s'est déroulée tous les cinq ans. Elle porte sur environ 430000 ménages, correspondant à peu près à 1100000 personnes de 15 ans et plus. À la 
différence des deux enquêtes précédentes, celle-ci n'adopte pas la convention du BIT, et est centrée sur l'état «habituel» de la situation professionnelle du répondant. Le questionnaire de 1956 comprend neuf questions au plus pour les personnes avec un travail, six questions pour les personnes sans travail et qui souhaitent travailler, et trois questions pour les personnes sans travail et qui ne désirent pas travailler. Le questionnaire de 2002 comporte, pour les personnes ayant une activité professionnelle, une trentaine de questions au plus, une vingtaine pour les personnes sans travail et cherchant un emploi, et une douzaine au plus pour les inactifs.

\section{Les catégories d'emploi dans les enquêtes}

La petite taille des questionnaires que nous venons de constater est certainement liée à la méthode de collecte. Toutefois, il ne suffit pas de réduire la taille du questionnaire pour rendre cette méthode opérationnelle. Il existe certes un manuel d'aide pour remplir le questionnaire, mais il ne peut pas remplacer l'enquêteur, censé préparer le répondant à l'enquête (5), l'aider à comprendre le sens des questions, et tenter d'éviter de possibles erreurs d'interprétation, etc. Plus fondamentalement, cette méthode implique que les catégories proposées aux répondants soient assez nombreuses et qu'il $\mathrm{y}$ ait une bonne adéquation des catégories statistiques avec les catégories du sens commun. Est-ce le cas? Si oui, comment cela fonctionne-t-il? Les catégories statistiques qui sont, par nécessité, rigoureuses et stabilisées (du moins est-ce leur vocation), peuvent-elles être suffisamment en adéquation avec les catégories de la vie quotidienne, et ceci sur une durée suffisamment longue, pour permettre l'analyse de l'évolution de l'emploi sur le long terme?

Partant ces questionnements, je vais à présent examiner les catégories d'emploi telles qu'elles apparaissent dans les questionnaires selon le système de classification adopté par les enquêtes.

Les catégories d'emploi proposées par les trois enquêtes(6) concernent le «statut d'emploi» (jyûgyô jô no chii) et la "forme d'emploi» (koyô keitai). Ces deux classifications sont fondées sur la déclaration du répondant, qui choisit parmi une liste de réponses.

\section{Le statut d'emploi}

Les éléments suivants figurent au début du questionnaire de la LFS.

Tableau 1 - Questionnaire de la LFS

Shigoto no naiyô : description détaillée du travail

- Remplissez la partie qui concerne le travail effectué durant la dernière semaine du mois (dans le cas du mois de décembre pour la période qui s'étend entre le 20 et le 26) ou le travail dont vous étiez temporairement absent durant la même semaine.

- Si vous avez effectué plus d'un travail, remplissez la partie qui concerne le travail pour lequel vous avez passé le plus d'heures.

\begin{tabular}{|c|c|c|}
\hline \multirow{6}{*}{$\begin{array}{l}\text { Jyûgyô jô no chii (statut d'emploi) } \\
\text { - Rinji yatoi no hito (temporaire) s'applique } \\
\text { aux personnes dont la durée du contrat est } \\
\text { d'un mois et plus, et inférieure à un an } \\
\text { - Hiyatoi no hito (journalier) s'applique aux } \\
\text { personnes dont la durée du contrat est infé- } \\
\text { rieure à un mois } \\
\text { - Jiei gyôshu (indépendant) signifie le proprié- } \\
\text { taire de magasin, l'exploitant agricole, etc. } \\
\text { à son propre compte } \\
\text { - Naishoku (travail domestique) signifie } \\
\text { le travail à la pièce exécuté à la maison }\end{array}$} & \multirow[t]{2}{*}{ Yatowarete iru hito no uchi : salarié } & $\begin{array}{l}\text { 1) Jôko no hito : salarié régulier } \\
\text { 2) Rinji yatoi no hito: temporaire } \\
\text { 3) Hiyatoi no hito : journalier }\end{array}$ \\
\hline & & $\begin{array}{l}\text { 4) Kaisha nado no yakuin: directeur } \\
\text { des firmes, etc. }\end{array}$ \\
\hline & \multirow{2}{*}{ Jiei gyôshu : indépendant } & 5) Yatoi nin ari : avec salariés \\
\hline & & 6) Yatoi nin nashi: sans salariés \\
\hline & & $\begin{array}{l}\text { 7) Jika eigyô no tetsudai : aide } \\
\text { de l'entreprise familiale indépendante }\end{array}$ \\
\hline & & 8) Naishoku : travail domestique \\
\hline
\end{tabular}

(5) L'enquêteur-distributeur de questionnaires présente l'enquête au répondant lors de son passage, mais il ne peut pas faire un travail relationnel de même qualité que l'enquêteur qui reste pour l'entretien. Par contre, la présence de celui-ci introduit le difficile problème des relations entre l'enquêteur et l'enquêté qui influenceraient les réponses.

(6) Voir glossaire en annexe. 
Comme nous pouvons le constater dans la colonne de gauche, le questionnaire ne donne aucune explication sur jôko(7) no hito, salarié régulier(8). La définition se construit par élimination, à partir des définitions des autres catégories de salariés. Ce caractère potentiellement non-substantiel de la catégorie d'emploi régulier (jôko) trouve son écho dans les modifications de sa définition. En effet, dans la terminologie utilisée dans les rapports de l'enquête LFS (terminologie inconnue des répondants), la définition de jôko se modifie au fil des années. De 1964 à 1970, jôkô est défini comme suit: «Personnes employées soit sans que soit fixée la durée d'embauche, soit pour une durée déterminée d'un an et plus, ainsi que directeurs des firmes, des associations et des organisations diverses». Entre 1971 et 1987, jôkô est défini comme "personnes autres que les rinji yatoi (temporaires) et hiyatoi (journaliers)». Entre 1988 et 1990, ce terme définit «les personnes employées sans détermination de durée ou pour une durée déterminée d'un an et plus». Entre 1991 et 1996 , cela devient «parmi les salariés, ceux qui sont autres que rinji yatoi et hiyatoi ». À partir de 1997, la définition est la suivante:

Tableau 2 - Définition de la catégorie Jôko à partir de 1997

\begin{tabular}{|l|l|l|}
\hline \multicolumn{2}{|l|}{ Salariés réguliers (jôko) dont } & \\
\hline Directeurs (yakuin) & $\begin{array}{l}\text { Directeurs des firmes, associations et organisations } \\
\text { diverses }\end{array}$ \\
\cline { 2 - 4 } & $\begin{array}{l}\text { Salariés réguliers ordi- } \\
\text { naires (ippan jôko) }\end{array}$ & $\begin{array}{l}\text { Personnes employées pour une durée d'un an et plus, } \\
\text { ou qui travaillent sur un contrat à durée indéterminée } \\
\text { (sauf les directeurs) }\end{array}$ \\
\hline $\begin{array}{l}\text { Salariés temporaires (rinji } \\
\text { yatoi) }\end{array}$ & $\begin{array}{l}\text { Personnes employées pour une durée déterminée d'un } \\
\text { mois et plus, et inférieure à un an }\end{array}$ \\
\hline Salariés journaliers (hiyatoi) & $\begin{array}{l}\text { Personnes employées sur une base journalière ou pour } \\
\text { une durée déterminée inférieure à un mois }\end{array}$ \\
\hline
\end{tabular}

Ce qui nous intéresse ici est le fait que, même dans la terminologie, la définition de jôko ne soit pas constante, et qu'à certaines périodes, ce soit une définition par défaut, à travers les définitions retenues pour les autres catégories d'emploi. Le fait que la catégorie d'emploi censée être au cœur du système soit définie via les autres catégories d'emploi mérite réflexion. Quant au questionnaire, l'enquêté doit procéder à une interprétation afin de savoir ce que c'est le jôko no hito, d'autant plus que jôko n'est pas un terme courant. Ce mot est l'abréviation de jôyô koyô, qui signifie emploi permanent, ce qui n'est absolument pas évident pour les personnes enquêtées. Ainsi que nous l'avons vu, ce terme était historiquement employé pour désigner les travailleurs permanents et les différencier des autres

(7) Peut être lu comme jôyatoi également.

(8) Traduit en anglais comme "regular employee» dans le rapport de l'enquête. $C f$. plus loin. travailleurs. De nos jours, il n'est pas utilisé dans la vie quotidienne. Par contre, rinji yatoi (temporaire) et hiyatoi (journalier) sont parfaitement compréhensibles (9).

Ainsi que nous le voyons dans la terminologie présentée ci-dessus, le Statistics Bureau donne comme définition du «salarié régulier ordinaire» des personnes employées pour une durée d'un an et plus, ou qui travaillent sur un contrat à durée indéterminée. Toutefois, dans la pratique, la plupart des «salariés réguliers ordinaires» n'ayant pas de contrat de travail, c'est plutôt l'opération par élimination qui prévaut.

Cenpendant, un problème se pose lorsque le questionnement est «qui sont ceux qui n'ont pas de contrat de travail dans l'entreprise?». C'est-àdire qui sont les personnes qui, dans la pratique sociale, entrent dans la catégorie de jôko, selon la définition donnée dans l'enquête? En effet, la plupart des travailleurs à temps partiel, les jeunes recrutés temporairement, c'est-à-dire les personnes qui seraient classées comme occupant les formes d'emploi dites atypiques, n'ont pas de contrat. Ainsi, de nos jours, la catégorie jôko, héritée de l'époque où jôkô koyô, l'emploi permanent, émergea en opposition à la catégorisation des travailleurs à mobilité très élevée ou des jeunes ouvrières dans le secteur de l'industrie légère, peut comprendre en son sein des formes d'emploi atypiques. Effectivement, l'Employment Status Survey (ESS ci-après) fournit un tableau croisé de la classification par statut et par forme d'emploi dans lequel figure le temps partiel, arubaito, le travail intérimaire, et le travail sous contrat dans la catégorie jôko comme dans les autres catégories. La SSLFS ainsi que l'ESS procèdent de la même manière que la LFS. Ni l'une ni l'autre ne fournissent la définition du «salarié régulier» (jôko) dans le questionnaire. Seule la SSLFS donne une définition dans le manuel d'aide pour remplir le questionnaire: «Jôko désigne la personne employée avec un contrat d'un an et plus ou avec un contrat à durée indéterminée».

Le caractère non rigoureux et potentiellement équivoque de la catégorie de jôko se retrouve également dans la traduction anglaise de ce terme utilisée dans les rapports d'enquêtes. erronée par rapport à la définition donnée par l'enquête. 
Traduction anglaise de jôko

\begin{tabular}{|c|c|c|}
\hline LFS & SSLFS & ESS \\
\hline Regular employee & Regular employee & Ordinary employee \\
\hline
\end{tabular}

Le même mot anglais, "Regular employee» apparaît dans la classification par la «forme d'emploi» (koyô keitai) (voir ci-dessous).

Traduction anglaise de seiki no shokuin, jyûgyôin

\begin{tabular}{|c|c|c|}
\hline LFS & SSLFS & ESS \\
\hline & Regular staff & Regular employee \\
\hline
\end{tabular}

Or, il s'agit de la traduction des termes seiki no shokuin, et jyûgyôin, "salarié typique», excluant donc les formes d'emploi atypiques (10). Comment le même terme peut-il désigner à la fois une catégorie incluant des formes d'emploi atypiques et une autre qui les exclut? Cette ambiguïté ou plutôt cette contradiction, est d'autant plus dérangeante que ces trois enquêtes partagent par ailleurs de nombreux concepts. Elle semble être révélatrice d'une accumulation, d'une stratification de significations successives à travers l'histoire. Afin d'examiner cette hypothèse, nous allons passer à présent au second système de classification.

\section{La forme d'emploi}

Dans le questionnaire de la SSLFS ainsi que dans le nouveau questionnaire spécial de la LSF comme dans l'ESS, figure une question aux répondants se déclarant comme salariés (à l'exclusion des directeurs), quant à leur appellation sur le lieu de travail. Les différentes appellations parmi lesquelles on leur demande de choisir sont identiques pour les deux derniers questionnaires.

Le questionnaire SSLFS ne donne pas de définition de différentes «formes d'emploi», ni dans le questionnaire lui-même, ni dans le manuel d'aide. En revanche, le questionnaire spécial de la LFS, qui a remplacé la SSLFS depuis 2002, fournit les définitions suivantes dans le manuel d'aide:
On peut remarquer que, tandis que les autres termes ne sont pas accompagnés de précision, seul haken shain est explicité déjà dans le questionnaire. Ce statut particulier de haken shain se retrouve dans le cas de l'ESS, qui fournit l'explication suivante:

\begin{tabular}{|l|}
\hline Question A1-2 : appellation sur le lieu de travail \\
\hline - Le haken shain, envoyé par l'agence de travail intéri- \\
maire » désigne les personnes encadrées par le Worker \\
Dispatching Law. \\
- Pour les salariés haken dans les magasins de grande \\
surface, ou les gardiens haken, référez-vous à l'appellation \\
qui était employée à l'agence intérimaire qui vous a envoyé.
\end{tabular}

De même, dans le manuel d'aide, l'ESS donne l'explication suivante:

- Si vous n'êtes pas sûr que vous êtes pâto ou arubaito, référez-vous à l'appellation qui a été utilisée dans l'annonce d'embauche ou dans les textes d'embauche ou encore lors du contrat d'embauche.

- Pour les personnes envoyées par l'agence de travail intérimaire, choisissez haken shain (intérimaire), même si d'autres appellations sont utilisées sur le lieu de travail.

L'explication concernant haken shain continue encore longuement dans le manuel ( $c f$. glossaire). Le fait que seule la catégorie haken shain fasse l'objet d'un long commentaire qui se réfère à la définition juridique suggère son caractère spécifique par rapport aux autres catégories d'emploi. D'où provient-t-il? Ces différentes formes d'emploi n'ont pas la même filiation historique. L'intérimaire et le contractuel sont plus récents; le premier est apparu dans le questionnaire en 1987, et le second en 2002, c'est-à-dire lors de la dernière enquête ESS. Les trois autres catégories ainsi que celle de «salarié ordinaire» sont des catégories plus anciennes. Le mot pâto taimu apparait pour la première fois dans le questionnaire ESS en 1968 comme une réponse - «Je souhaite être embauché à temps réduit (par exemple pâto taimu)» - à la question «Quelle sorte de travail souhaitez-

\begin{tabular}{ll}
\hline \multicolumn{2}{l}{ Question A4 : concernant votre travail actuel, si vous êtes salarié, indiquez l'appellation utilisée sur le lieu du travail } \\
\hline $\begin{array}{l}\text { Questionnaire } \\
\begin{array}{l}\text { Seiki no shokuin, } \\
\text { jyûgyôin }\end{array}\end{array}$ & Explication donnée dans le manuel d'aide \\
\hline $\begin{array}{l}\text { Pâto }{ }^{1} \text {, arubaito } 2 \\
\text { S'applique aux personnes appelées ippan shokuin (salarié ordinaire) ou seishain (salarié titulaire), etc. }\end{array}$ \\
$\begin{array}{l}\text { Haken shain (envoyé } \\
\text { par l'agence de travail } \\
\text { intérimaire) }\end{array}$ & $\begin{array}{l}\text { S'appliquent aux personnes qui sont appelées ainsi ou d'une manière similaire sur le lieu de travail sans } \\
\text { Dispatching Law }{ }^{3} \text {. Si votre cas correspond à cette catégorie, choisissez celle-ci, même si vous êtes appelé } \\
\text { par un autre terme listé ici }\end{array}$ \\
\hline Keiyaku shain & $\begin{array}{l}\text { S'applique aux personnes qui sont engagées avec un contrat à durée déterminée pour occuper un poste à } \\
\text { une compétence spécifique }\end{array}$ \\
\hline $\begin{array}{l}\text { Shokutaku } 4 \\
\text { S'applique aux personnes appelées ainsi ou de manière similaire sur leur lieu de travail, sans tenir compte } \\
\text { des conditions de travail ou de la durée du contrat }\end{array}$ \\
\hline
\end{tabular}

1 Le terme pâto taimu et son abréviation pâto sont des néologismes à partir de l'anglais «part time » (temps partiel).

2 Mot dérivé de l'allemand « arbeit », - travail.

3 Rôdôsha hakenhô.

4 Les personnes à qui on « confie » (shokutaku, traduit comme « entrust » en anglais) le travail. 
vous»? Dans la liste de réponses, on voit figurer, à côté de pâto, la réponse suivante: «Je souhaite être embauché avec un horaire normal - futsû kinmu).

La question concernant l'appellation sur le lieu de travail figurant dans le second système de classification est apparue en 1982. Les quatre réponses proposées sont: seiki no shokuin/jyûgyôin, pâto/arubaito, shokutaku etc., autres. Elles ont été introduites avant la mise en œuvre de la politique de flexibilisation du marché de l'emploi. L'intégration de l'intérimaire en 1987 et du contractuel en 2002 marque l'entrée d'un langage juridique dans ce système de classification qui était jusqu'alors fondé uniquement sur la dénomination ordinaire, plus précisément sur la pratique langagière de l'entreprise. Ainsi, dans le même système de classification, différentes couches de langage de codification peuvent s'accumuler. Les explications dans le manuel d'aide montrent bien que la cohabitation entre ces deux langages n'est pas encore stabilisée, et que le sens à donner aux mots n'est pas partagé de manière homogène par les salariés. L'histoire de la SSLFS est à peu près parallèle: le terme pâto taimu apparaît en 1967; en 1981, parmi les réponses figurent pour la première fois seiki jyûgyôin ainsi que arubaito, marquant la formalisation concomitante de ces catégories. Quant à la question concernant l' «appellation sur le lieu de travail», elle entre dans le questionnaire SSLFS en 1983 lorsque figure pour la première fois shokutaku. L'intérimaire apparaît en 1999, et le contractuel a été intégré en 2002.

Nous avons vu que la désignation de la plupart des formes d'emploi n'est pas fondée sur le contrat de travail, car, dans la grande majorité des cas, il n'existe pas de contrat légal. Normalement il devrait exister un règlement intérieur d'entreprise définissant les statuts et les formes d'emploi, mais souvent il n'y en a pas ou s'il y en a un, ce règlement est souvent caduc. De manière générale, l'emploi au Japon est relativement peu régulé par un cadre juridique. En lieu et place d'un système légal relevant des pouvoirs publics et dépassant ainsi le niveau de l'entreprise, il revient à cette dernière d'encadrer le statut de l'emploi(11). Cela ne signifie pas que l'entreprise soit libre de disposer de ses salariés comme elle l'entend. Cette pratique d'emploi fondée sur un consensus social concernant le rôle de l'entreprise dans la société est effectivement sanctionnée par la société et protégée par la jurisprudence(12) (Sugeno, 2004).

(11) Le cadre législatif existe, bien entendu, mais d'un côté, il laisse beaucoup plus d'espace à l'entreprise comparativement à ce qui se passe en France, et de l'autre côté, il est amendé par une nombre important de dérogations.

(12) C'est bien pour cette raison que la vague de licenciements massifs dans les grandes entreprises a suscité une angoisse au sein de la société japonaise. Non seulement du fait de l'importance numérique de ces licenciements, mais plus encore parce que le consensus social ne tient plus. Il s'agit bien de l'effritement d'une norme.
Si c' est une logique juridique qui donne de la cohérence aux deux formes les plus récentes d'emploi, quelle est la logique qui sous-tend les quatre autres formes? Ces formes d'emploi plus anciennes, «titulaire», «temps partiel», «arubaito», «shokutaku», pour lesquels les questionnaires n'expliquent pratiquement rien, ce qui laisse supposer une multiplicité de sens, sont très fortement liées au genre et au cycle de vie. Le temps partiel concerne les femmes de plus de 35 ans, c'est-à-dire celles qui reviennent sur le marché de l'emploi après avoir élevé leurs enfants. L'arubaito est le fait des jeunes, garçons et filles. En fait, il s'agit de «petits boulots». Les pâto aussi bien que les arubaito sont payés à l'heure, et sont exclus des avantages sociaux accordés par l'entreprise. Le shokutaku est lié aux hommes âgés de plus de 55 ans, et surtout de plus de 65 ans. Dans la pratique, il s'agit d'hommes qui continuent de travailler au sein de l'entreprise après avoir pris leur retraite, en tant que salariés titulaires. Enfin la notion de «titulaire» est fortement liée aux hommes adultes ( $c f$. tableau 3 ).

Ainsi, c'est la construction sociale des formes d'emploi, intégrant les relations de genre, d'âge, le rôle de l'institution familiale et la division sexuelle du travail, qui sous-tend la cohérence de ce système de classification. Cette construction sociale fournit la base de l'adéquation entre les catégories ordinaires et les catégories statistiques, et ainsi étaie la validité opérationnelle lors de la collecte d'information.

\section{Changements historiques et tensions entre catégories d'emploi}

Au-delà de l'opérationnalité des catégories d'emploi, le fait que ce système de classification savant se base sur les pratiques de dénomination au sein de l'entreprise donne une bonne indication sur la place de l'entreprise dans la société japonaise, à côté de l'État et d'autres institutions publiques : bien des éléments pris en charge par les pouvoirs publics en France sont, au Japon, dévolus à l'entreprise avec l'accord des partenaires sociaux. Le consensus social s'est longtemps appuyé sur le fait que l'entreprise assurait la stabilité à long terme des revenus des ménages à travers le salaire du chef de famille travaillant en son sein. Ce consensus structure les catégories d'emploi en les mettant en relation avec les différents attributs sociologiques de l'individu, aussi bien du point de vue du genre que de la place du salarié dans le cycle de vie. Les formes d'emploi, «typique» et «atypique» par exemple, et leurs appellations expriment aussi bien la position de l'individu que la place de l'entreprise dans cette configuration.

Les deux langages, dans le second système de classification fondé sur la forme d'emploi, reflètent les tensions entre les deux champs, celui de la loi et celui de l'entreprise. Ces tensions semblent 
Tableau 3

Répartition des emplois selon leur forme

en 2001 (en \%)

\begin{tabular}{|c|c|c|c|c|c|c|c|c|}
\hline & & $\begin{array}{l}\text { Tous } \\
\text { âges }\end{array}$ & $\begin{array}{c}15-24 \\
\text { ans }\end{array}$ & $\begin{array}{c}25-34 \\
\text { ans }\end{array}$ & $\begin{array}{c}35-44 \\
\text { ans }\end{array}$ & $\begin{array}{c}45-54 \\
\text { ans }\end{array}$ & $\begin{array}{c}55-64 \\
\text { ans }\end{array}$ & $\begin{array}{l}65 \text { ans } \\
\text { et plus }\end{array}$ \\
\hline \multirow{9}{*}{$\begin{array}{l}\stackrel{\mathscr{U}}{\Xi} \\
\stackrel{\Xi}{\Xi} \\
\stackrel{\Xi}{\Xi}\end{array}$} & Directeurs & 9 & 0 & 2 & 7 & 11 & 19 & 32 \\
\hline & Seiki no shokuin, jyûgyôin & 78 & 64 & 90 & 89 & 83 & 62 & 26 \\
\hline & Emploi atypique & 13 & 35 & 8 & 4 & 6 & 19 & 42 \\
\hline & Pâto & 2 & 2 & 1 & 0 & 1 & 3 & 12 \\
\hline & Arubaito & 5 & 29 & 3 & 1 & 1 & 3 & 8 \\
\hline & Intérimaire & 0 & 1 & 0 & 0 & 0 & 0 & 1 \\
\hline & Contractuel et shokutaku & 4 & 3 & 2 & 2 & 2 & 10 & 16 \\
\hline & Autres & 1 & 1 & 1 & 1 & 1 & 3 & 5 \\
\hline & Total emploi & 100 & 100 & 100 & 100 & 100 & 100 & 100 \\
\hline \multirow{9}{*}{ 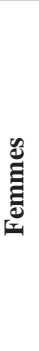 } & Directeurs & 4 & 0 & 1 & 4 & 6 & 8 & 32 \\
\hline & Seiki no yakuin, jyûgyôin & 51 & 57 & 66 & 47 & 43 & 39 & 25 \\
\hline & Emploi atypique & 45 & 43 & 33 & 49 & 51 & 53 & 41 \\
\hline & Pâto & 30 & 8 & 18 & 37 & 42 & 43 & 29 \\
\hline & Arubaito & 7 & 27 & 6 & 4 & 2 & 3 & 3 \\
\hline & Intérimaire & 2 & 1 & 3 & 2 & 1 & 0 & 0 \\
\hline & Contractuel et shokutaku & 5 & 5 & 4 & 4 & 4 & 5 & 5 \\
\hline & Autres & 2 & 1 & 2 & 2 & 1 & 2 & 5 \\
\hline & Total emploi & 100 & 100 & 100 & 100 & 100 & 100 & 100 \\
\hline
\end{tabular}

Sources: Special Survey of the Labour Force Survey.

augmenter dans la situation actuelle de crise où l'Etat tente de revenir sur le devant de la scène afin de prendre en charge les fonctions délaissées par les entreprises défaillantes.

Je voudrais revenir à présent sur le caractère, que j'ai qualifié de non-substantiel, du statut du salarié régulier (jôko) et sur le premier système de classification, basé sur le statut d'emploi. De même que l'ambiguïté et la tension dans le second système de classification relèvent du contexte historique, dans le premier système, le contexte historique met bien en évidence le caractère équivoque du statut du salarié régulier. En effet, lors de démarrage de ces enquêtes, les formes d'emploi qualifiées d'atypiques aujourd'hui, notamment le pâto (temps partiel), l'arubaito et le shokutaku n'étaient pas encore développées. Cela ne signifie pas que la population associée à ces formes d'emploi n'était pas présente sur le marché du travail. Prenons le cas des femmes. L'évolution du taux d'activité au Japon montre que les femmes japonaises maintiennent un taux d'activité assez stable ( $c f$. figure 1). Le changement s'observe plutôt dans la progression de la salarisation, en parallèle avec la forte diminution de l'aide familiale, en particulier dans le secteur de l'agriculture et de la forêt ( $c f$. tableau 4). Le travail à temps partiel apparaît vers le milieu des années 1950, son développement remonte aux années
1970, mais surtout après les années 1980. Lors des premières périodes des enquêtes sur la force du travail examinées ici, le brouillage de la catégorie jôko du fait de l'intégration des autres catégories d'emploi émergentes ne s'est pas encore produit. Au début, dans les années 1950 et sans doute dans la grande partie des années 1960, les trois statuts de salariés, jôko, temporaire et journalier étaient probablement aptes à appréhender le salariat.

Figure 1

Évolution du taux d'activité au Japon entre 1955 et 2005

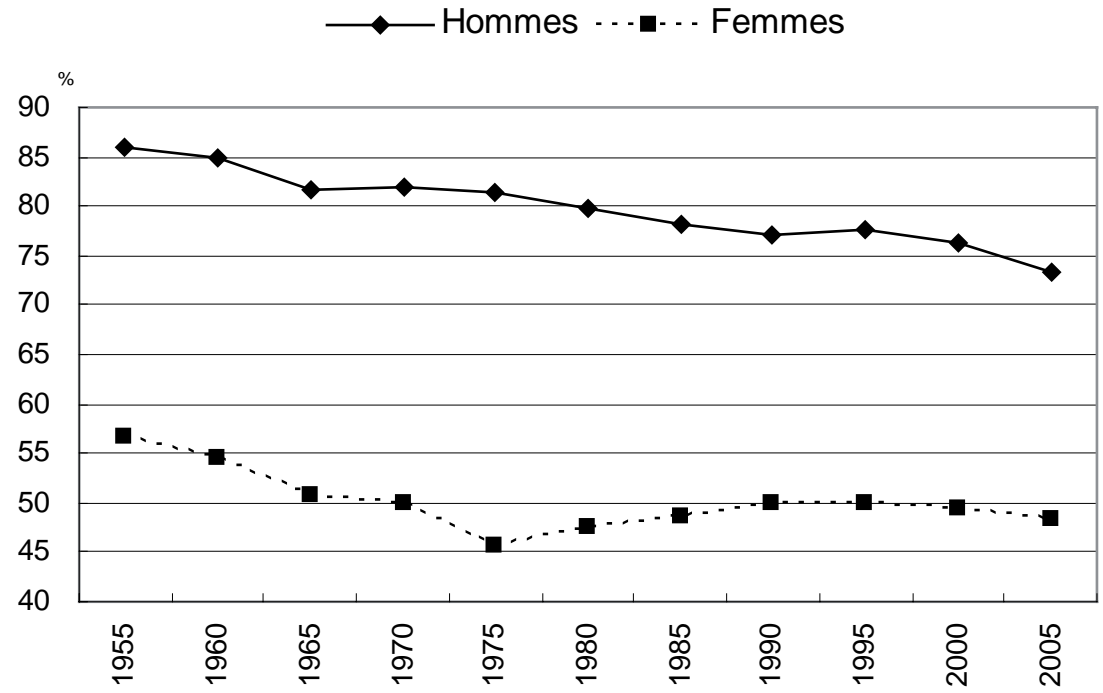

Sources: Labour Force Survey.

Champs : 15 ans et plus. 
Tableau 4

Évolution de la salarisation par sexe au Japon entre 1955 et 2005.

\begin{tabular}{|c|c|c|c|c|c|c|}
\hline & \multicolumn{3}{|c|}{ Hommes } & \multicolumn{3}{|c|}{ Femmes } \\
\hline & 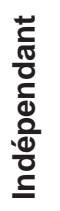 & 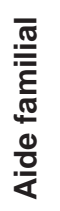 & $\begin{array}{l}\frac{0}{\frac{1}{2}} \\
\frac{\pi}{\sqrt[T]{D}}\end{array}$ & 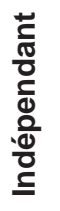 & 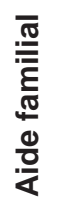 & $\frac{0}{\text { ஸे }}$ \\
\hline 1955 & 32 & 16 & 52 & 16 & 53 & 31 \\
\hline 1960 & 27 & 11 & 62 & 16 & 43 & 41 \\
\hline 1965 & 23 & 8 & 69 & 15 & 37 & 49 \\
\hline 1970 & 22 & 6 & 71 & 14 & 31 & 55 \\
\hline 1975 & 20 & 4 & 76 & 14 & 26 & 60 \\
\hline 1980 & 19 & 3 & 77 & 14 & 23 & 63 \\
\hline 1985 & 18 & 3 & 79 & 13 & 20 & 67 \\
\hline 1990 & 16 & 3 & 81 & 11 & 17 & 72 \\
\hline 1995 & 14 & 2 & 84 & 9 & 13 & 78 \\
\hline 2000 & 14 & 2 & 84 & 8 & 11 & 81 \\
\hline 2005 & 13 & 2 & 85 & 6 & 9 & 85 \\
\hline
\end{tabular}

Sources: Labour Force Survey.

Note:En 2005, 85\% des hommes occupés actifs sont salariés, $13 \%$ sont indépendants et $2 \%$ aides familiaux.

Champ: actifs occupés de 15 ans et plus.
On a remédié à l'effritement de la catégorie jôko et à l'insuffisance du premier système de classification fondé sur le statut d'emploi par l'introduction, en 1983, du second système de classification fondé sur la forme d'emploi et prenant mieux en compte la salarisation croissante et la diversification des formes d'emploi. Les catégories du premier système correspondaient à des périodes pendants lesquelles la part des indépendants et des aides familiaux demeurait importante. Dans le second système, les catégories sont issues des pratiques empiriques dont la sédimentation, appuyée par la jurisprudence, a assuré, du moins pour une certaine période, la stabilisation des catégories d'emploi.

Ainsi les catégories d'emploi sont sujettes aux changements, et les épaisseurs accumulées au fil du temps peuvent les rendre équivoques.

Il est évident que des comparaisons internationales appellent la mise en évidence préalable des contextes nationaux historiques et institutionnels dans lesquels les catégories ont émergé et ont évolué. La même précaution est nécessaire au niveau national, car les mêmes catégories d'emploi peuvent recouvrir des réalités différentes selon l'époque, le secteur d'activité, ou encore tout simplement selon l'enquête. Cette vigilance est d'autant plus indispensable que les épaisseurs de signification demeurent souvent implicites et inconscientes. 


\section{Bibliographie}

Desrosières A. (2000), «L'histoire de la statistique comme genre: styles d'écriture et usages sociaux», Genèses, 39.

Desrosières A. (2001), «Entre réalisme métrologique et conventions d'équivalence: les ambiguïtés de la sociologie quantitative», Genèses, 43.

Desrosières A. (2003), «Comment fabriquer un espace de commune mesure? Harmonisation des statistiques et réalisme de leurs usages », in: M. Lallement et J. Spurk (éds), Stratégies de la comparaison internationale, Paris, CNRS éditions.

Desrosières A., Thevenot L. (1988), Les catégories socioprofessionnelles, Paris, La Découverte.

Douglas M. (1986), How institutions think, New York, Syracuse University Press, (traduction française) Ainsi pensent les institutions, éditions Usher, 1989 et Comment pensent les institutions, Paris, La Découverte. 2004.

Douglas M., Hill D. éd. (1992), How classification works, Edinburgh, Edinburgh University Press.

Fus H. (1995), Nihongata kigyô shakai to josei rôdô. Shokugyô to katei no ryôritsu o mezashite, Kyoto, Minerva shobô.

Gordon A. (1988), The evolution of labour relations in Japan. Heavy Industry, 1853-1955, Council of East Asian Studies, Harvard University, Cambridge \& London.

Hazama H. (1976), "Formation of An Industrial Work Force: Historical Changes in the Life Style of Industrial Workers", in: Hugh Patrick (ed.), Japanese Industrialization and Its Social Consequences, Berkeley, University of California Press.

Hazama H. (1978), Nihon rômu kanri kenkyû, Ochanomizu shobô, Tokyo.

Hirata H., Sugita K. (1988), «Politique paternaliste et division sexuelle du travail: le cas de l'industrie japonaise», Le Mouvement Social, n 144.

IwaI H. (1992), Rôdôryoku, koyô, shitsugyô tôkei no kokusaiteki tenkai, Chiba, Azusa shuppan.

Kase K., Sugita K. éd. (2006), The Unemployed and Unemployment in an International Perspective: Comparative Studies of Japan, France and Brazil, ISS Research Series, Institut des Sciences Sociales, Université de Tokyo, Tokyo.
Magaud J., Sugita K. (1993), «Le retour des réseaux : à propos d'une comparaison franco-japonaise», Gérer et Comprendre, $\mathrm{n}^{\circ} 31$.

Magota R. (1978), Nenkô chingin no shûen, Nikkei shinsho, Tokyo.

Nomura M. (1994), Shûshin koyô, Iwanami, Tokyo.

Ôкаwа T. (1979), Dekasegi no keizaigaku, Kinokuniya, Tokyo.

SAкамото F. (1977), Nihon koyôshi volume 2. Nenkôsei heno nagai michinori, Chûôkeizaisha, Tokyo.

Sugeno K. (2004), Shin koyô shakai no hô, Yûhikaku, Tokyo.

Sugita K. (1989), «Le Japon: jeux de miroirs », Sociologie du travail, $\mathrm{n}^{\circ} 2$.

Sugita K (1997), «Sous-traitance en cascade au Japon: le cas d'un groupe d'entreprises dans le secteur électronique », Précarisation sociale, travail et santé, IRESCO/ CNRS.

Sumiya M, Kobayashi K., Hyodo Ts. (1967), Nihon shihonshugi to rôdô mondai, Tokyo daigaku shuppankai, Tokyo.

SumiYa M. (1976), Nihon chinrôdô no shiteki kenkyû, Ochanomizu shobô, Tokyo.

SumiYa M. éd. (1977), Nihon shokugyô kunren hattenshi, 2 vol., Nihon rôdô kyôkai, Tokyo.

Thomann B. (2005), «Japon. Les emplois non réguliers et les mécanismes de la régulation sociale», Chronique internationale de l'IRES, $\mathrm{n}^{\circ} 97$.

Thomann B. (2005), «Les conditions historiques de la naissance et de la reproduction de l'emploi à vie comme archétype de l'emploi masculin au Japon », Le Mouvement Social, $\mathrm{n}^{\circ} 210$.

Urabe K., Ômura K. (1983), Nihon teki rôshi kankei no tankyû, Chûô keizaisha, Tokyo.

Watanabe S., Haneda A. (1977), Dekasegi rôdô to nôson no seikatsu, Tokyo daigaku shuppankai, Tokyo.

Watanabe S. (1987), Dekasegi no sôgôteki kenkŷ̂, Tokyo daigaku shuppankai, Tokyo. 


\section{Glossaire}

La traduction anglaise provient de rapport annuel ou du site internet du Statistic Bureau (les mots clés sont traduits en français par l'auteure). En l'absence d'une traduction officielle en anglais, une traduction française est fournie à partir du texte japonais.

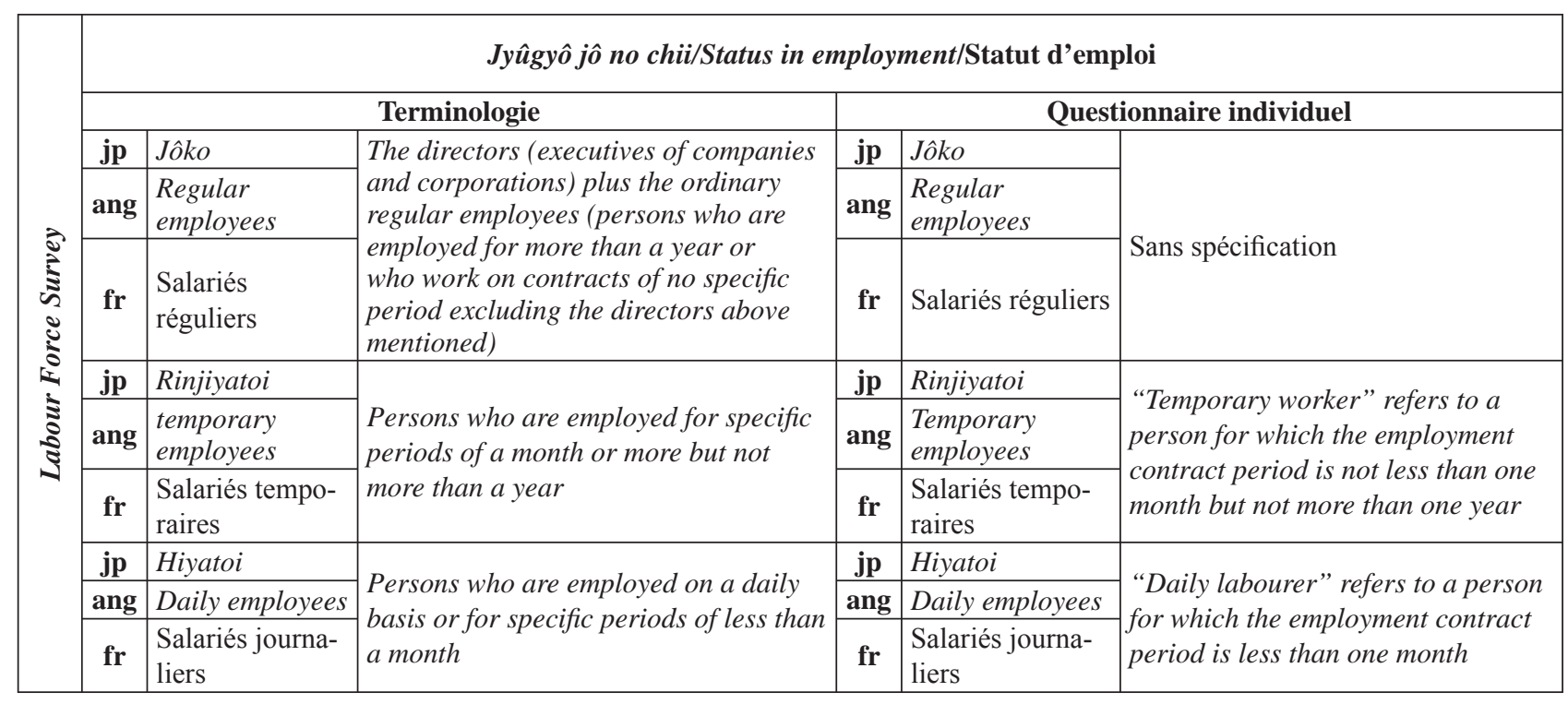

\begin{tabular}{|c|c|c|c|c|c|c|}
\hline \multirow{11}{*}{ 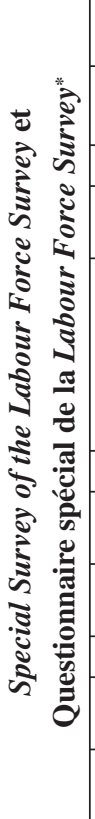 } & \multicolumn{6}{|c|}{ Jyûgyô jô no chii/Status in employment/Statut d'emploi } \\
\hline & \multicolumn{3}{|r|}{ Terminologie } & \multicolumn{3}{|c|}{$\begin{array}{l}\text { Questionnaire individuel et manuel d'aide } \\
\text { pour remplir le questionnaire }\end{array}$} \\
\hline & jp & Jôko & \multirow{3}{*}{$\begin{array}{l}\text { Executives of company/corporation } \\
\text { (Executives, managing directors } \\
\text { and auditors of private companies, } \\
\text { associations, public corporations, etc.) } \\
\text { and ordinary employees, ippan jôko, } \\
\text { (persons who work on contracts of no } \\
\text { specific period or a year or more of } \\
\text { employment excluding executives of } \\
\text { company/corporation above mentioned }\end{array}$} & jp & Jôko & \multirow[b]{3}{*}{$\begin{array}{l}\text { Personne employée pour une durée } \\
\text { d'un an et plus, ou qui travaillent sur } \\
\text { un contrat à durée indéterminée }\end{array}$} \\
\hline & ang & $\begin{array}{l}\text { Regular } \\
\text { employees }\end{array}$ & & ang & $\begin{array}{l}\text { Regular } \\
\text { employee }\end{array}$ & \\
\hline & fr & $\begin{array}{l}\text { Salariés } \\
\text { réguliers }\end{array}$ & & fr & Salarié régulier & \\
\hline & jp & Rinjiyatoi & \multirow{3}{*}{$\begin{array}{l}\text { Persons who work on contracts of a } \\
\text { month or more but not more than a year }\end{array}$} & jp & Rinjiyatoi & \multirow{3}{*}{$\begin{array}{l}\text { Personne employée pour une durée } \\
\text { d'un an et plus, ou qui travaillent sur } \\
\text { un contrat à durée indéterminée }\end{array}$} \\
\hline & ang & $\begin{array}{l}\text { Temporary } \\
\text { employees }\end{array}$ & & ang & $\begin{array}{l}\text { Temporary } \\
\text { employee }\end{array}$ & \\
\hline & fr & $\begin{array}{l}\text { Salariés } \\
\text { temporaires }\end{array}$ & & fr & $\begin{array}{l}\text { Salarié } \\
\text { temporaire }\end{array}$ & \\
\hline & jp & Hiyatoi & \multirow{3}{*}{$\begin{array}{l}\text { Persons who work on a daily basis or on } \\
\text { contracts of less than a month }\end{array}$} & jp & Hiyatoi & \multirow{3}{*}{$\begin{array}{l}\text { Personne employée sur une base } \\
\text { journalière ou pour une durée } \\
\text { déterminée inférieure à un mois }\end{array}$} \\
\hline & ang & $\begin{array}{l}\text { Daily } \\
\text { employees }\end{array}$ & & ang & Daily labourer & \\
\hline & $\mathbf{f r}$ & $\begin{array}{l}\text { salariés } \\
\text { journaliers }\end{array}$ & & $\mathbf{f r}$ & Salarié journalier & \\
\hline
\end{tabular}

* Aucune spécification quant à la koyô keitai, forme d'emploi, dans la SSLFS. 


\begin{tabular}{|c|c|c|c|c|c|c|}
\hline \multirow{20}{*}{ 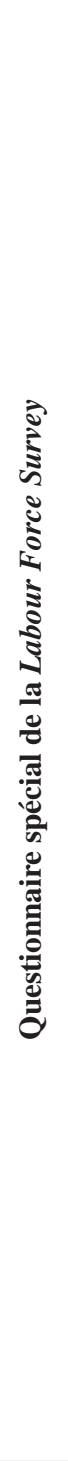 } & \multicolumn{6}{|c|}{ Koyô keitai/Form of employment/forme d'emploi (According to how their position is called at their workplace) } \\
\hline & \multicolumn{3}{|c|}{ Terminologie } & \multicolumn{3}{|c|}{ Manuel d'aide pour remplir le questionnaire } \\
\hline & jp & $\begin{array}{l}\text { Seiki no shokuin, } \\
\text { jyûgyôin }\end{array}$ & \multirow{3}{*}{$\begin{array}{l}\text { Sans } \\
\text { spécification }\end{array}$} & jp & \begin{tabular}{|l} 
Seiki no shokuin, \\
jyûgyôin
\end{tabular} & \multirow{3}{*}{$\begin{array}{l}\text { S'applique aux personnes appelées ippan shokuin } \\
\text { (salarié ordinaire) ou seishain (salarié titulaire), etc. }\end{array}$} \\
\hline & ang & Regular staff & & ang & Regular employee & \\
\hline & fr & Salarié régulier & & fr & Salarié régulier & \\
\hline & jp & Pâto & \multirow{3}{*}{$\begin{array}{l}\text { Sans } \\
\text { spécification }\end{array}$} & jp & Pâto & \multirow{6}{*}{$\begin{array}{l}\text { S'appliquent aux personnes qui sont appelées ainsi } \\
\text { ou d'une manière similaire sur le lieu de travail sans } \\
\text { qu'on tienne compte de leur horaire de travail ni du } \\
\text { nombre de jours travaillés }\end{array}$} \\
\hline & ang & Part-time worker & & ang & Part-time worker & \\
\hline & $\mathrm{fr}$ & \begin{tabular}{|l|} 
Travailleur à \\
temps partiel
\end{tabular} & & $\mathbf{f r}$ & \begin{tabular}{|l|} 
Travailleur à \\
temps partiel
\end{tabular} & \\
\hline & jp & Arubaito & \multirow{3}{*}{$\begin{array}{l}\text { Sans } \\
\text { spécification }\end{array}$} & jp & Arubaito & \\
\hline & \begin{tabular}{|l|} 
ang \\
\end{tabular} & Arubaito & & ang & Arubaito & \\
\hline & $\mathrm{fr}$ & Arubaito & & fr & Arubaito & \\
\hline & jp & $\begin{array}{l}\text { Rôdôsha haken } \\
\text { jigyôsho no haken } \\
\text { shain }\end{array}$ & \multirow{3}{*}{$\begin{array}{l}\text { Sans } \\
\text { spécification }\end{array}$} & jp & $\begin{array}{l}\text { Jinzai haken kigyô } \\
\text { no haken shain }\end{array}$ & \multirow{3}{*}{$\begin{array}{l}\text { S'applique aux personnes envoyées par une agence } \\
\text { de travail intérimaire dans le cadre du Worker } \\
\text { Dispatching Law. Si votre cas correspond à cette } \\
\text { catégorie, choisissez celle-ci même si vous êtes } \\
\text { appelé par un autre terme listé ici }\end{array}$} \\
\hline & ang & $\begin{array}{l}\text { Dispatched } \\
\text { workers from } \\
\text { temporary labour } \\
\text { agency }\end{array}$ & & ang & $\begin{array}{l}\text { Worker dispatched } \\
\text { from a dispatching } \\
\text { service agency }\end{array}$ & \\
\hline & fr & $\begin{array}{l}\text { Intérimaire envoyé } \\
\text { par l'agence de } \\
\text { travail intérimaire } \\
\end{array}$ & & $\mathrm{fr}$ & $\begin{array}{l}\text { Intérimaire envoyé } \\
\text { par l'agence de } \\
\text { travail intérimaire }\end{array}$ & \\
\hline & jp & $\begin{array}{l}\text { Keiyaku shain, } \\
\text { shokutaku }\end{array}$ & \multirow{3}{*}{$\begin{array}{l}\text { Sans } \\
\text { spécification }\end{array}$} & jp & \begin{tabular}{|l}
$\begin{array}{l}\text { Keiyaku shain, } \\
\text { shokutaku }\end{array}$ \\
\end{tabular} & \multirow{3}{*}{$\begin{array}{l}\text { Contractuel s'applique aux personnes qui sont } \\
\text { engagées avec un contrat à durée déterminée pour } \\
\text { occuper un poste à une compétence spécifique. } \\
\text { Shokutaku s'applique aux personnes appelées ainsi } \\
\text { ou de manière similaire sur leur lieu de travail, sans } \\
\text { tenir compte des conditions de travail ou de la durée } \\
\text { du contrat }\end{array}$} \\
\hline & ang & $\begin{array}{l}\text { Contract employee } \\
\text { or entrusted } \\
\text { employee }\end{array}$ & & ang & $\begin{array}{l}\text { Contract employee } \\
\text { or entrusted } \\
\text { employee }\end{array}$ & \\
\hline & fr & $\begin{array}{l}\text { Salarié contractuel } \\
\text { ou salarié à qui on } \\
\text { confie le travail }\end{array}$ & & fr & $\begin{array}{l}\text { Salarié contractuel } \\
\text { ou salarié à qui on } \\
\text { confie le travail }\end{array}$ & \\
\hline & jp & Sonota & \multirow{3}{*}{$\begin{array}{l}\text { Sans spécifi- } \\
\text { cation }\end{array}$} & jp & Sonota & \multirow{3}{*}{ Sans spécification } \\
\hline & ang & Other & & ang & Other & \\
\hline & fr & Autres & & fr & Autres & \\
\hline
\end{tabular}

\begin{tabular}{|c|c|c|c|c|c|c|}
\hline \multirow{11}{*}{ 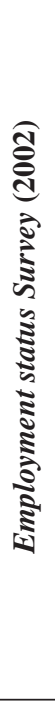 } & \multicolumn{6}{|c|}{ Jyûgyô jô no chii/Status in employment/Statut d'emploi } \\
\hline & \multicolumn{3}{|r|}{ Terminologie } & \multicolumn{3}{|r|}{ Questionnaire individuel } \\
\hline & jp & Ippan jôko & \multirow{3}{*}{$\begin{array}{l}\text { Employees other than executives, } \\
\text { "Temporary employees" and } \\
\text { "Daily employees" }\end{array}$} & jp & Jôko & \multirow{3}{*}{ Sans spécification } \\
\hline & ang & $\begin{array}{l}\text { Ordinary } \\
\text { employees }\end{array}$ & & ang & $\begin{array}{l}\text { Ordinary } \\
\text { employee }\end{array}$ & \\
\hline & fr & \begin{tabular}{|l|}
$\begin{array}{l}\text { Salariés } \\
\text { réguliers }\end{array}$ \\
\end{tabular} & & $\mathbf{f r}$ & \begin{tabular}{|l|}
$\begin{array}{l}\text { Salarié } \\
\text { régulier }\end{array}$ \\
\end{tabular} & \\
\hline & jp & Rinjiyatoi & \multirow{3}{*}{$\begin{array}{l}\text { Those who are employed on a } \\
\text { contract of employment for a term } \\
\text { of a month or more but less than } \\
\text { a year }\end{array}$} & jp & Rinjiyatoi & \multirow{6}{*}{$\begin{array}{l}\text { Temporary employee» means a worker with an } \\
\text { employment contract of } 1-12 \text { months. "Daily } \\
\text { employee" means a worker with an employ- } \\
\text { ment contract of less than a month. }\end{array}$} \\
\hline & ang & $\begin{array}{l}\text { Temporary } \\
\text { employees }\end{array}$ & & ang & \begin{tabular}{|l} 
Temporary \\
employee
\end{tabular} & \\
\hline & fr & \begin{tabular}{|l|} 
Salariés \\
temporaires
\end{tabular} & & fr & \begin{tabular}{|l|} 
Salarié \\
temporaire
\end{tabular} & \\
\hline & jp & Hiyatoi & \multirow{3}{*}{$\begin{array}{l}\text { Those who are employed on a } \\
\text { daily basis or on a contract of } \\
\text { employment for a term of less than } \\
\text { a month. }\end{array}$} & jp & Hiyatoi & \\
\hline & ang & \begin{tabular}{|l|}
$\begin{array}{l}\text { Daily } \\
\text { employees }\end{array}$ \\
\end{tabular} & & ang & \begin{tabular}{|l|}
$\begin{array}{l}\text { Daily } \\
\text { employee }\end{array}$ \\
\end{tabular} & \\
\hline & fr & \begin{tabular}{|l|}
$\begin{array}{l}\text { Salariés } \\
\text { journaliers }\end{array}$ \\
\end{tabular} & & fr & \begin{tabular}{|l|}
$\begin{array}{l}\text { Salarié } \\
\text { journalier }\end{array}$ \\
\end{tabular} & \\
\hline
\end{tabular}




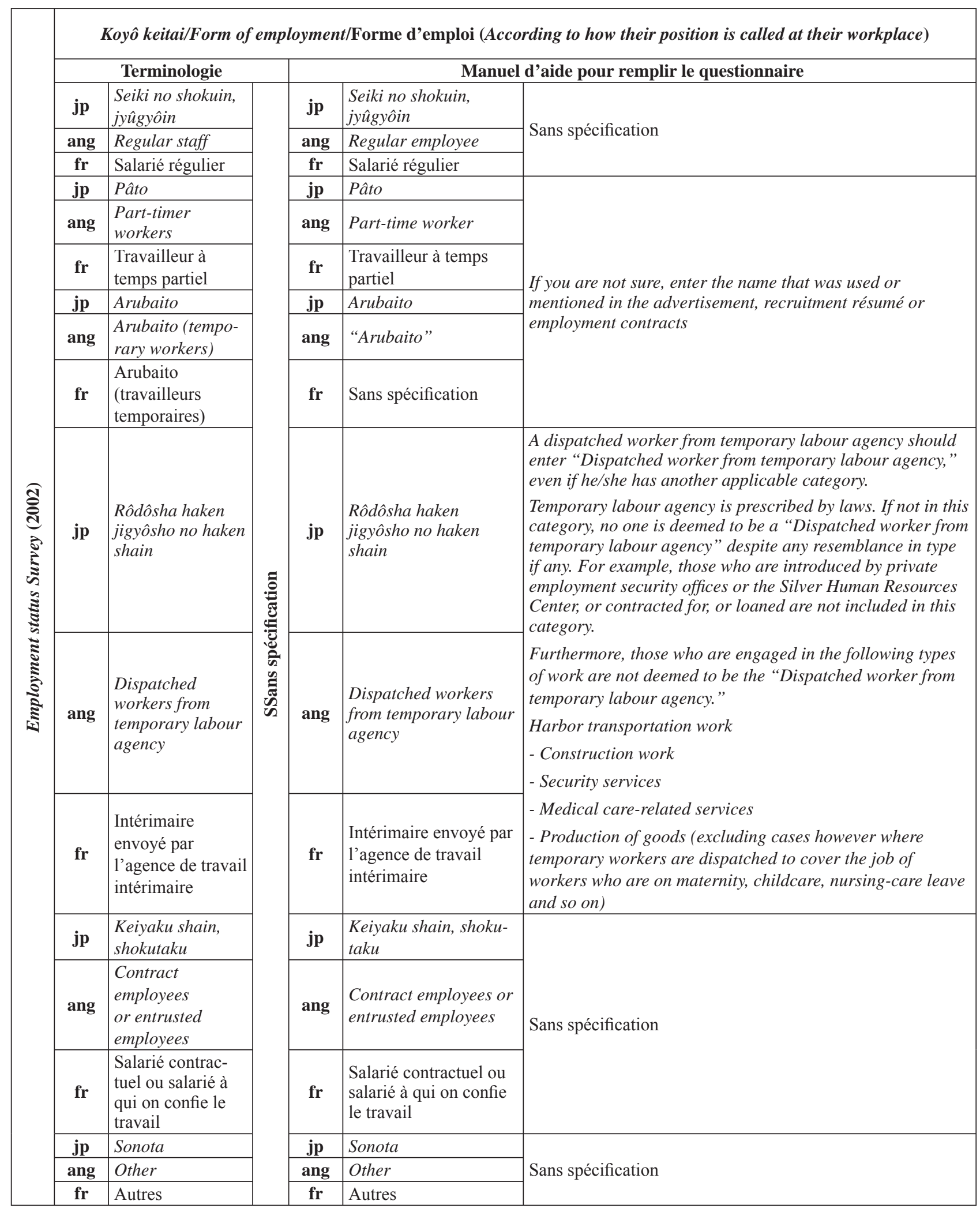




\title{
Les fusions théâtrales dans les nouveaux Länder
}

\author{
Héritage culturel, fardeau financier
}

Laure de Verdalle $\left(^{*}\right)$

La réunification politique des deux Allemagnes a eu de fortes incidences sur l'organisation du travail théâtral dans les nouveaux Länder. À partir d'un socle historique commun (la République de Weimar), les deux Allemagnes ont développé durant quarante années des politiques publiques théâtrales très différentes, pour ne pas dire opposées : tandis qu'un processus de rationalisation du tissu théâtral a été mis très tôt en æuvre en RFA, aboutissant à une relative concentration des lieux scéniques, la RDA s'est caractérisée au contraire par une grande densité de structures "multigenres ». Dès le début de la transition, c'est le modèle ouest-allemand qui semble imposer ses normes. Les villes moyennes des nouveaux Länder doivent regrouper à marche forcée leurs structures culturelles afin de réaliser des économies d'échelle. L'article étudie ces processus de fusion tant du point de vue matériel et politique que de celui de la production culturelle et de l'organisation du travail artistique. Mais loin de confirmer la prédominance du modèle fédéral ouest-allemand sur les structures théâtrales de l'exAllemagne de l'Est, les processus de rationalisation économique et administrative dans la sphère culturelle se traduiraient par un «effet en retour» du modèle dominé sur le modèle dominant.

En 1990, lors de la réunification des deux Allemagnes, la RDA présente un paysage théâtral public d'une exceptionnelle densité. Une soixantaine d'Ensembles se partagent 173 lieux scéniques et emploient près de 17000 personnes, toutes activités confondues. À titre de comparaison, la RFA compte alors 88 théâtres publics (pour une population quatre fois supérieure) employant 28000 personnes (1). Les nouveaux Länder se distinguent également de leurs homologues de l'Ouest par la répartition géographique de leurs institutions théâtrales publiques dont $42 \%$ sont situées dans des villes de moins de 50000 habitants, contre $7 \%$ seulement au sein des anciens Länder (2). Enfin, les théâtres publics de 1'Est sont majoritairement des maisons «multigenres » (Mehrspartentheater), qui regroupent sous un même toit théâtre, orchestre, opéra et parfois même ballet.

(*) Chargée de recherche CNRS. Laboratoire «Printemps», Guyancourt : laure.de-verdalle@printemps.uvsq.fr.

(1) À l'Est comme à l'Ouest, les théâtres publics fonctionnent selon le modèle de l'ensemble, qui réunit une troupe fixe de comédiens, metteurs en scène, chanteurs, etc. ainsi qu'un personnel technique et administratif permanent. Mais la RDA a généralisé en 1972 l'utilisation du contrat à durée indéterminée au sein de ses théâtres alors que la RFA fonctionne avec des contrats à durée déterminée d'un ou deux ans.

(2) Berlin n'est pas prise en compte dans cette comparaison.
Ces différences, qui se sont construites à partir d'un héritage commun(3), doivent être rapportées aux politiques culturelles et artistiques menées durant quarante ans par les deux États allemands. Tandis que la RFA, par des stratégies de concentration et des réformes structurelles, a entamé assez tôt la rationalisation de son tissu théâtral public, la RDA, en partie grâce à une politique culturelle fortement centralisée, a garanti la survie des institutions théâtrales publiques dispersées sur l'ensemble de son territoire.

En 1990, le tissu théâtral est-allemand se trouve brutalement confronté à un modèle de gestion publique des affaires culturelles importé de l'Ouest. Pour les théâtres de l'ex-RDA, la transition se traduit notamment par le passage d'un financement centralisé à un financement décentralisé de la culture (LABORIER, 1992). La vie culturelle est en effet un des domaines dans lesquels s'exprime le mieux le principe du fédéralisme ouest-allemand : ce sont les villes et les Länder qui supportent la charge financière de la majeure partie des équipements culturels(4). Dans

(3) Celui de la République de Weimar qui avait consolidé un modèle théâtral reposant sur une municipalisation de la culture, autour d'institutions publiques.

(4) En 1989, les dépenses culturelles de la RFA se sont élevées à 9 milliards de DM: $55 \%$ de cette somme provient des communes, $38 \%$ des Länder et seulement $7 \%$ du Bund (WIESAND, 1992). 
les anciens Länder, la taille moyenne d'une ville susceptible d'entretenir un Mehrspartentheater et son ensemble est de 200000 habitants(5). On comprend dès lors les difficultés rencontrées par les villes estallemandes pour conserver leur institution théâtrale après la réunification, et ce d'autant plus qu'elles se heurtent à d'importantes difficultés économiques et sociales. Dans ce contexte, on observe tout au long des années 1990 une progressive remise en cause du paysage théâtral est-allemand, qui prend toutefois des formes différentes en fonction des stratégies adoptées par les Länder de l'Est et par leurs municipalités (Verdalle (DE), 2002). C'est l'une de ces stratégies, la fusion théâtrale, que nous nous proposons d'étudier ici car elle incarne bien l'exigence de mise aux normes ouest-allemandes du paysage théâtral hérité de l'ex-RDA. En effet, la fusion est un instrument de rationalisation du tissu théâtral qui répond à l'introduction de nouveaux modèles de gestion des affaires culturelles. Toutefois, les cas qui seront étudiés ici sont ceux de fusions opérant entre des Ensembles est-allemands. La question traitée sera donc moins celle des difficultés du management interculturel(6) (BARMEYER, 2005) que celle de la restructuration d'un paysage théâtral et des organisations qui le composent face aux contraintes (notamment financières) introduites par la réunification.

Pour analyser les conséquences de ce choc, nous commencerons par rappeler rapidement quelques caractéristiques générales de la réunification et de ses effets en matière de politique culturelle. Puis nous étudierons différents cas de fusions théâtrales menées en Saxe et en Thuringe, en montrant qu'au-delà d'un cadre commun (la réunion de deux Ensembles publics et le partage de leurs lieux scéniques), plusieurs options ont été mobilisées, qui font intervenir des rapports de force entre les municipalités et entre les institutions théâtrales concernées. Nous pourrons alors analyser les conséquences locales de ces fusions sur l'organisation du travail théâtral, sur les modalités de la production et sur l'état des relations au public. Nous conclurons en interrogeant l'impact à moyen/long terme de ces fusions (notamment en matière de droit du travail).

(5) En ce qui concerne les trois Länder que j'ai étudiés dans le cadre de ma thèse de doctorat, seule la Saxe présente trois villes qui remplissent cette condition (Dresde, Leipzig et Chemnitz). En Thuringe, Erfurt, la capitale du Land atteint à peine ce plancher. Et dans le Brandebourg, Potsdam, la plus grande ville, compte seulement quelque 120000 habitants.

(6) Même si certaines entités issues des fusions ont été confiées à la gestion d'intendants venus de l'Ouest.

\section{Encadré 1 \\ Méthodologie}

Ce texte s'appuie sur les résultats d'une thèse de sociologie soutenue en octobre 2003 et consacrée à la recomposition du monde théâtral est-allemand après la réunification. Cette recherche visait à étudier conjointement : 1) l'action des politiques publiques sur le remodelage du tissu théâtral des nouveaux Länder; 2) les transformations organisationnelles qui s'opèrent au sein des théâtres publics durant les années 1990 ; et 3) les parcours professionnels des gens de théâtre originaires de l'ex-RDA.

En ce qui concerne plus particulièrement les fusions, l'enquête s'est concentrée sur les Länder de Saxe et de Thuringe. En effet, tous deux ont eu recours aux fusions dans les années 1990 et présentaient, au moment de la réunification, des paysages théâtraux relativement similaires et extrêmement denses. Par ailleurs, ces deux Länder se caractérisaient par la forte présence d'institutions théâtrales publiques dans des petites villes de moins de 50000 habitants (même si la Saxe compte également deux grandes villes de plus de 500000 habitants, Dresde et Leipzig).

Quatre cas de fusion ont fait l'objet d'une analyse approfondie :

\begin{tabular}{|c|c|c|c|c|}
\hline & $\begin{array}{c}\text { Altenburg/ } \\
\text { Gera } \\
\text { (Thuringe) }\end{array}$ & $\begin{array}{l}\text { Eisenach/ } \\
\text { Rudolstadt } \\
\text { (Thuringe) }\end{array}$ & $\begin{array}{c}\text { Freiberg/ } \\
\text { Döbeln } \\
\text { (Saxe) }\end{array}$ & $\begin{array}{c}\text { Plauen/ } \\
\text { Zwickau } \\
\text { (Saxe) }\end{array}$ \\
\hline $\begin{array}{l}\text { Date } \\
\text { de la fusion }\end{array}$ & 1995 & 1995 & 1993 & 2000 \\
\hline $\begin{array}{l}\text { Nombre } \\
\text { d'habitants }\end{array}$ & $\begin{array}{c}\text { Altenburg: } \\
46000 \\
\text { Gera: } \\
126000\end{array}$ & $\begin{array}{c}\text { Eisenach : } \\
46000 \\
\text { Rudolstadt : } \\
29000\end{array}$ & $\begin{array}{c}\text { Freiberg: } \\
48000 \\
\text { : Döbeln : } \\
25000\end{array}$ & $\begin{array}{r}\text { Plauen: } \\
68000 \\
\text { Zwickau } \\
105000\end{array}$ \\
\hline $\begin{array}{l}\text { Distance } \\
\text { séparant les } \\
\text { deux villes }\end{array}$ & $35 \mathrm{~km}$ & $90 \mathrm{~km}$ & $36 \mathrm{~km}$ & $42 \mathrm{~km}$ \\
\hline
\end{tabular}

Chacun de ces cas a donné lieu à une monographie, construite à partir des données du Deutsche Bühnenverein (1), antérieures et postérieures à la fusion (budgets, effectifs employés, nombre de mises en scène produites, nombre de représentations dans les différents lieux scéniques, taux de fréquentation, etc.). Des entretiens ont été conduits au sein des Ensembles, afin de revenir sur les changements organisationnels consécutifs aux fusions, mais aussi dans les ministères de la Culture ainsi que dans les services culturels des municipalités concernées, afin de saisir les enjeux locaux des fusions et les stratégies mises en œuvre par chaque Land dans la restructuration de son paysage théâtral.

(1) Cette association des scènes allemandes, créée en 1846, s'intéresse à toutes les questions de politique culturelle relatives au théâtre et à son organisation. Elle publie chaque année des statistiques détaillées. 


\section{Le passage d'une gestion centralisée à une gestion décentralisée de la culture}

Le traité d'unification, signé en août 1990, entérine l'extension du modèle fédéral et administratif ouest-allemand à la partie Est de l'Allemagne. En effet, la réalisation de l'unité allemande par le biais de l'article 23 de la Loi Fondamentale impose la réorganisation du territoire est-allemand en Länder et sa mise aux normes ouest-allemandes. La loi sur la formation des nouveaux Länder(7) prend effet le 14 octobre 1990 et ses conséquences dans le domaine des politiques culturelles sont immédiates : le ministère est-allemand de la Culture est démantelé et le traité d'unification opère un transfert des compétences en matière de gestion et de financement de la culture vers les Länder et les communes. Des ministères régionaux compétents pour les arts, la culture et l'éducation sont mis en place.

Mais si la réunification des deux Allemagnes intervient rapidement, un an à peine après la chute du mur, la renaissance de ces entités territoriales (8) et leur autonomisation suivent un processus marqué par plusieurs étapes. Dans le domaine de la culture, le Bund est amené à jouer un rôle transitoire important, contrevenant par là même à sa tradition de nonintervention dans les affaires culturelles des Länder. Le traité d'unification comprend ainsi une clause culturelle (l'article 35), qui concerne la sauvegarde de la «substance culturelle» est-allemande. Ce principe de conservation conduit le Bund à financer plusieurs programmes d'aide exceptionnels, qui atteignent un total de 900 millions de DM pour l'année 1991 et de 2,9 milliards de DM jusqu'en 1994 (LABORIER, 1996). Toutefois, ces aides se tarissent assez rapidement et à partir de 1994 le Bund se fait plus discret.

Les nouveaux Länder restent alors seuls face au modèle décentralisé de gestion des affaires culturelles et doivent définir leurs propres stratégies de financement. Pour cela, ils vont s'inspirer de ce qui existe dans les anciens Länder (le principe des partenariats administratifs entre anciens et nouveaux Länder favorise la transmission des expériences), tout en profitant de la plasticité inhérente au fédéralisme. La comparaison de deux cas, celui de la Saxe et celui de la Thuringe ( $c f$. encadrés 2 et 3 ), permet de comprendre comment les choix mis en œuvre s'inscrivent dans des situations économiques et politiques locales ainsi que dans des histoires régionales et urbaines.

(7) Saxe, Thuringe, Saxe-Anhalt, Brandebourg et Mecklembourg Poméranie Occidentale.

(8) La RDA avait supprimé les Länder en 1952.
Encadré 2

\section{La Saxe ou la défense d'une politique autonome}

Dans les années qui suivent la réunification, la Saxe exploite largement l'image d'une tradition culturelle et d'une identité locale fortes et anciennes. Assez proche en cela de la Bavière (à l'Ouest), ce Land affirme sa spécificité au sein des nouveaux Länder en inventant des mécanismes originaux permettant d'amortir la transition. La loi sur les espaces culturels saxons, adoptée en décembre 1993, vise ainsi à réorganiser les relations entre les différents niveaux d'administration locale afin d'assurer dans la durée le financement des institutions culturelles. Chacun des espaces est pensé comme une «coopérative», mettant en commun des ressources financières pour soutenir la vie culturelle.

Mais ce dynamisme est hypothéqué par la nécessaire coordination entre les différents niveaux administratifs et par la fragilité financière des communes saxonnes, qui rendent difficile la gestion du dispositif basé sur un principe de solidarité. La loi de 1993 apparaît surtout comme une tentative d'adaptation défensive du Land et des communes face aux exigences introduites par le traité d'unification. La fusion qui intervient en 2000 entre les théâtres de Plauen et de Zwickau ouvre la voie à une restructuration radicale (bien que retardée) du paysage théâtral saxon. En 2002, une nouvelle fusion réunit les théâtres de Bautzen et de Zittau et la même année, le maire de Dresde, avec le soutien du ministère de la Culture du Land, propose de fusionner l'opérette et le Staatsschauspiel, envisageant même à plus long terme de réunir toutes les scènes publiques de la ville sous un même conseil d'intendance. Ce dernier projet soulève l'opposition farouche du milieu théâtral, mais ne semble pas totalement abandonné.

La Saxe et la Thuringe ont donc l'une et l'autre recours aux fusions pour restructurer leur paysage théâtral dans les années qui suivent la réunification. Cette stratégie, présentée comme une alternative aux fermetures de théâtres, est toutefois ponctuée d'hésitations et de nombreux retournements d'alliance. Pour comprendre ces tâtonnements, regardons à présent comment ces fusions fonctionnent concrètement et quelles sont les difficultés qu'elles posent, à différents niveaux. L'analyse des conséquences de la réunification dans le domaine théâtral rejoint ici un questionnement plus large portant sur l'extension des modèles gestionnaires à la sphère culturelle. 


\section{Encadré 3 \\ La Thuringe ou la crise du modèle ouest-allemand de financement de la culture}

La Thuringe se conforme plus fidèlement au modèle ouest-allemand de financement de la culture et se heurte très vite à la question de la rationalisation d'un paysage théâtral particulièrement dense. Dès le début des années 1990, le Land a construit sa politique théâtrale à partir d'une carte, raisonnant en termes de régions et d'espaces de spectateurs (Zuschauerraum) :

«Prenons le public qui vit ici [il désigne un point sur la carte du Land, proche de la ville de Sonderhausen] : où se trouve pour lui la possibilité la plus proche d'aller au théâtre? Erfurt est à $75 \mathrm{~km}$, et aujourd'hui encore, c'est un temps de trajet de près de 45 minutes. Ou Eisenach, mais c'est à $80 \mathrm{~km}$ ». Directeur du théâtre au ministère de la Culture du Land de Thuringe, Erfurt, avril 2002.

Le principe des fusions est rapidement mis en avant par le ministère de la Culture du Land pour préserver une offre théâtrale diversifiée sur l'ensemble du territoire. Il s'agit de restructurer en profondeur un tissu théâtral fortement concentré dans des villes de petite et moyenne importance (en 1991, cinq des huit théâtres du Land se trouvent dans des villes de moins de 50000 habitants). Une première vague de fusions d'orchestres a lieu dès 1992. Une seconde vague, en 1995, fait cette fois intervenir des fusions entre théâtres (Altenburg et Gera d'une part, Rudolstadt et Eisenach de l'autre). À partir de 2000-2001, une troisième étape s'amorce, et prévoit de regrouper trois entités qui sont elles-mêmes le résultat de fusions antérieures (le théâtre d'Eisenach/Rudolstadt, celui de Nordhausen/ Sonderhausen et la philharmonie de GothaSuhl). Après de multiples tergiversations, le ministère de la Culture de Thuringe fait finalement marche arrière. En 2003, la fusion entre Eisenach et Rudolstadt (problématique depuis l'origine en raison de la distance séparant les deux villes) est cassée. Les deux théâtres retrouvent leur autonomie mais sont sommés de nouer de nouveaux partenariats : Rudolstadt semble aujourd'hui avoir accepté l'idée d'un rapprochement avec Nordhausen, tandis qu'Eisenach envisage une coopération avec Meinigen.

\section{Des négociations difficiles}

Une fusion entraine la création d'une nouvelle entité théâtrale qui se présente de la manière suivante : un seul Ensemble disposant de plusieurs lieux scéniques, dans différentes villes (deux pour commencer, les deux partenaires de la fusion). Pour parveniràcerésultat, et puisqueles théâtres concernés sont presque toujours des Mehrspartentheater, la modalité la plus fréquente est celle d'un partage des genres : l'une des villes hérite du théâtre (Schauspiel) et l'autre de l'opéra (Musiktheater). Toutes deux conservent un ensemble à demeure (les comédiens d'un côté, les solistes, l'orchestre et le chœur de l'autre), et les genres tournent ensuite d'une ville à l'autre au grès des représentations. L'objectif est de maintenir une offre théâtrale diversifiée, tout en réduisant les coûts de production qui sont partagés entre les villes partenaires de la fusion.

\section{Le poids des luttes d'influence}

Mais si les municipalités concernées par les fusions se retrouvent autour d'un objectif d'économies d'échelle, être cogestionnaire de ces nouvelles entités ne va pas de soi. Différents facteurs doivent être pris en compte : 1'histoire des villes et de leur théâtre, le rôle traditionnel du Stadttheater dans l'espace urbain en Allemagne, les vieilles rivalités opposant certaines communes, etc. Ainsi, lors de la fusion entre les théâtres de Freiberg et de Döbeln, dans la Saxe, le profil très différent des deux villes a joué (et joue encore) un rôle essentiel. Freiberg, avec ses 46000 habitants, est une ville bourgeoise, qui abrite une petite université et qui s'enorgueillit de posséder l'un des plus beaux théâtres de Saxe (une réplique en miniature du célèbre Semperoper de Dresde). Döbeln, qui ne compte que 23000 habitants, n'a pas les mêmes traditions théâtrales et a été sévèrement touchée par la crise économique qui sévit au sein des nouveaux Länder. À cela s'ajoute que Freiberg se situe dans la zone d'influence de Dresde, ville d'art et de culture, tandis que Döbeln est plus proche de Leipzig, ville commerçante.

«Döbeln fait partie de la zone d'influence de Leipzig, très nettement. [...] Les gens de Freiberg au contraire se sentent proches de Dresde. Dresde est par tradition une ville de culture. [...] Il y a toujours eu des villes de théâtre en RDA, c'est ainsi. Freiberg et Dresde sont des villes avec une riche tradition théâtrale à l'Est, comme Meiningen ou Weimar. Il $\mathrm{y}$ a des villes de théâtre et il y a les autres, qui n'en sont pas». Comédien, Mittelsächsiches Theater, Freiberg, décembre 2001.

Et dans tous les cas, les négociations sont mouvementées, illustrées par des revirements, des jeux d'alliance et de contre-alliance, qui font intervenir les intendances des différents théâtres, les représentants des municipalités, mais aussi plus directement 
les habitants. La fusion qui a lieu en 2001 entre Plauen et Zwickau témoigne bien de ce processus complexe. En effet, dès 1993, l'intendant de Zwickau évoque l'idée d'un Westsächsiches Landestheater, qui réunirait les théâtres de Zwickau, Plauen et Annaberg. Mais Plauen et Annaberg refusent, par crainte de s'engager dans un processus qui serait dominé par Zwickau. En 1996, la municipalité de Zwickau reprend l'initiative. Mais entre-temps Plauen a ouvert des négociations avec Hof, une petite ville de Bavière (9). La distance entre Plauen et Hof est inférieure à celle qui sépare Plauen de Zwickau et par ailleurs Hof ne compte que 51000 habitants (contre 104000 pour Zwickau), ce qui rassure les représentants de Plauen (70 000 habitants). Mais les habitants de Hof se mobilisent contre le projet en créant une initiative citoyenne (Bürgerinitiative): brandissant la menace d'un boycott, ils parviennent à faire reculer leurs représentants. Devant ces multiples tergiversations, le ministère de la Culture de la Saxe lance finalement un ultimatum à Plauen et Zwickau et contraint les deux villes à une fusion rapide (le contrat est signé en 1998 et la fusion est effective en 2000). Tout en étant un acteur essentiel de la rationalisation du paysage théâtral public, le Land est ici soucieux de se placer au-dessus des conflits engendrés par les processus de fusion.

«Dans le cas de la fusion entre Plauen et Zwickau, [...] cela s'est finalement terminé dans mon bureau et là, il a fallu déployer beaucoup de patience pour ménager les susceptibilités. [...] Parfois, c'est ainsi, malgré la nécessité évidente de la fusion, nécessité financière, cette nécessité pour les théâtres n'est pas reconnue ou même niée. C'est un problème que nous n'avons pas encore résolu». Ministre de la Culture du Land de la Saxe, Dresde, avril 2001.

\section{Des fusions rarement équilibrées}

Les craintes qui se manifestent à travers ces jeux d'alliance sont d'autant plus compréhensibles qu'une fusion est rarement équilibrée, et fait intervenir des partenaires inégaux. Même avec un partage des genres, l'une des deux villes s'impose toujours comme le centre de gravité de la nouvelle entité. La localisation de la nouvelle intendance est d'ailleurs un enjeu fort au moment des négociations. La ville qui compte le plus d'habitants est généralement en position dominante (par exemple Gera, Eisenach, Zwickau et Freiberg dans les cas qui nous intéressent).

Mais c'est surtout dans la répartition des postes entre les deux ensembles que le caractère inégalitaire de la fusion se manifeste. En effet, la fusion des ensembles s'accompagne généralement d'une réduction globale des effectifs et il est souvent

(9) Plauen se situe dans le Vogtland, une zone de la Saxe qui est frontalière de la Bavière. facile de distinguer parmi les deux ensembles d'origine qui en ressort gagnant. Ainsi, pour le théâtre d'Altenburg-Gera, l'Ensemble de Gera se maintient à $67 \%$ après la fusion, celui d'Altenburg à $45 \%$ seulement. Gera, qui conserve le Musiktheater, voit ses solistes relativement préservés (douze chanteurs sur dix-neuf et dix chanteuses sur treize restent dans le nouvel ensemble), tandis qu'Altenburg doit se séparer de sept de ses douze chanteurs et de sept de ses dix chanteuses. Mais la contrepartie n'est pas tout à fait vraie pour le Schauspiel (qui revient à Altenburg) : certes, Altenburg sauve une partie de ses comédiens (cinq hommes sur douze et six femmes sur dix), mais Gera garde douze de ses seize comédiens et cinq de ses dix comédiennes.

\section{Les conséquences organisationnelles des fusions}

Deux enjeux importants se dessinent lorsque l'on considère les conséquences à court et moyen terme des fusions sur les organisations théâtrales. Le premier concerne la mise en œuvre du travail théâtral et les contraintes nouvelles qui émergent, pour l'institution et pour ses personnels, en raison de la multiplication des lieux scéniques. Le second est lié à l'ancrage traditionnel des théâtres publics allemands dans des villes et des histoires culturelles locales. La fusion, notamment parce qu'elle brouille les mécanismes d'identification qui unissent l'institution à son public, vient ici encore contraindre l'organisation de la production théâtrale.

\section{La recomposition du travail théâtral sous de nouvelles contraintes}

« Les hommes politiques croient souvent de façon un peu rapide que la simple addition de six petits orchestres en engendre un grand très bon. C'est une idée idiote, une idée de comptable ». August Everding, président du Deutsche Bühnenverein, in Theater der Zeit, 11/1991, p. 44.

Comment à partir de plusieurs théâtres n'en faire qu'un? Comment gérer les nouvelles contraintes organisationnelles nées de ce rapprochement et de la multiplication des lieux scéniques ? Ainsi peut se résumer le problème de la fusion des Ensembles, tel qu'il se pose dans le cadre du Stadttheater allemand. En effet, comme le souligne un éditorial de la $G D B A(10)$, « la réunion de deux théâtres existants n'accroît pas nécessairement l'efficacité artistique. $\mathrm{Au}$ contraire : les contraintes supplémentaires que l'on fait peser sur les personnes à travers l'obliga-

(10) Genossenschaft Deutscher Bühnen Angehöriger, syndicat créé en 1871 et qui représente les membres des professions artistiques du théâtre en Allemagne fédérale. À l'heure actuelle, cette organisation regroupe environ 7000 adhérents. 
tion de se déplacer ont des conséquences sur leur travail et sur les productions. Les temps de répétition pour travailler les œuvres se raccourcissent et sont de plus en plus compliqués à organiser. Du côté de la technique, il est nécessaire de créer des possibilités de déplacement. Les transports spéciaux pour les décors, les bus pour le personnel, tout ce qui doit être déplacé entraîne des coûts supplémentaires » (Herdelin, 1993). Plusieurs éléments doivent donc être pris en compte pour évaluer l'impact des fusions sur l'activité théâtrale des Ensembles : la distance entre les lieux scéniques, les contraintes techniques et les modes de réorganisation du travail théâtral.

Les fusions ont généralement lieu entre des villes assez proches (une trentaine de kilomètres), mais on trouve des exceptions, puisque Rudolstadt et Eisenach sont distantes de 90 kilomètres. Les difficultés liées au transport des décors et à leur démontage sont toutefois communément partagées, et ce d'autant plus que les théâtres des nouveaux Länder restent, après la fusion, des théâtres de répertoire : les spectacles sont présentés en alternance durant toute la saison dans les différents lieux scéniques. Diverses stratégies sont néanmoins mises en œuvre pour tenter de minimiser les déplacements de décors d'une ville à l'autre. Par exemple, une pièce jouée en alternance dans une ville pendant la première moitié du mois se déplacera ensuite dans l'autre ville pour la deuxième quinzaine (la rotation peut aussi se faire sur le principe d'un mois sur deux, voir même d'un semestre sur deux). Mais ces formes de stabilisation sont toutes relatives. Pour certaines pièces, une rotation rapide est impérative. C'est le cas notamment entre novembre et janvier pour les contes de Noël, qui doivent être représentés simultanément dans les deux villes, ce qui accroît la charge de travail des équipes techniques. À cela s'ajoute le problème de la compatibilité des scènes sur lesquelles l'ensemble se produit en alternance. De coûteux travaux sont parfois nécessaires pour permettre l'alignement des espaces scéniques sur des normes communes.

Pour les comédiens (ou les chanteurs), la fusion entraîne de nouvelles conditions de travail et une nouvelle gestion des ressources humaines. En effet, les fusions imposent aux membres des ensembles une forte mobilité, qu'ils doivent souvent gérer par eux-mêmes (les théâtres n'organisent que rarement des transports collectifs et les membres des ensembles doivent alors prendre en charge leurs déplacements dans le cadre des répétitions et des représentations). La réorganisation des emplois du temps, générée par la multiplication des lieux scéniques, est donc une contrainte forte pour les membres des ensembles, même si la présence de plusieurs lieux scéniques permet aux comédiens de jouer une même pièce un plus grand nombre de fois et plus longtemps.
«Le transport des comédiens de chez eux aux différents lieux de répétition a été à lui tout seul une exigence très forte et a engendré les plus grandes difficultés. La plupart de ceux qui ont demandé des ruptures de contrat volontaires [...] l'ont fait à cause de ce problème. On a essayé au départ de mettre en place des dédommagements financiers, mais on s'est vite rendu compte que ce n'était pas faisable. L'intendant a alors décidé que des transports seraient organisés pour les répétitions générales et pour les premières. Pour toutes les autres représentations, il faut se débrouiller tout seul». Responsable des relations au public, théâtre de Plauen-Zwickau, Zwickau, décembre 2001.

«Pour nous la fusion signifiait donc [...] que nous allions aussi jouer à Döbeln et que les deux maisons seraient donc nos lieux de travail. Cela implique bien sûr des contraintes, parce que nous sommes responsables de nos déplacements, que nous effectuons avec notre voiture privée. [...] C'est contraignant, parce qu'il arrive souvent que l'on ait une répétition tôt ici à Freiberg, puis encore un petit quelque chose dans l'après-midi, et que le soir nous jouions à Döbeln, et ensuite il faut que nous rentrions chez nous [à Freiberg]. Donc c'est une charge». Comédienne, Mittelsächsiches Theater, Freiberg, décembre 2001.

«Par exemple, nous pouvons avoir deux répétitions à Altenburg dans la même journée, la première de 10 heures à 14 heures, et la seconde en fin d'aprèsmidi. Mais que peut-on faire là-bas dans l'intervalle [il habite à Gera] ? Le temps de trajet, c'est une chose qui n'est finalement pas si grave. Par contre, ensuite, on est coincé là-bas». Comédien, théâtre d'Altenburg-Gera, Gera, avril 2002.

En interne, la fusion est donc vécue comme un pis-aller qui certes garantit pour un temps la survie du théâtre, mais qui n'est accepté que faute de mieux et qui est vécu sous le mode de la contrainte. Ces réticences s'observent lors des négociations préalables aux fusions, qui s'accompagnent souvent de manifestations ou de pétitions organisées par les Ensembles concernés. Mais surtout les fusions entraînent parfois des scissions volontaires. Ainsi, au moment de la fusion entre Eisenach et Rudolstadt (Eisenach conserve le Musiktheater et Rudolstadt le Schauspiel), qui se caractérise, rappelons-le, par la grande distance qui sépare les deux villes, des comédiens ont refusé le transfert du Schauspiel à Rudolstadt et ont choisi de créer leur propre troupe, sous un statut associatif, pour continuer de jouer à Eisenach.

\section{Les enjeux de l'ancrage local}

Une caractéristique importante du Stadttheater allemand est d'être ancré dans un environnement local et d'adapter son offre à un public donné. Des difficultés surgissent donc dès qu'il s'agit d'élaborer une même programmation pour deux villes 
dont les caractéristiques sociales et culturelles sont parfois très différentes. Par ailleurs, les rancœurs provoquées par le processus de fusion ont pu, dans certains cas, attiser les tensions. Les comédiens sont très sensibles à ce problème et l'évoquent fréquemment en situation d'entretien.

«À Döbeln, nous ne trouvons pas une grande résonance. Si l'on considère le potentiel de spectateurs, c'est une ville qui n'a pas d'écoles supérieures, pas d'universités, pas d'entreprises. [...] Nous avons peu de spectateurs là-bas, et c'est dommage, parce que nous proposons la même qualité. [...] Nous ne jouons pas autre chose là-bas, il n'y a pas un deuxième répertoire. [...] J'ai fait " $x$ " mises en scène à Döbeln, et ça a toujours été extraordinairement difficile de faire quelque chose avec le public. Ce qui ici [à Freiberg] a du succès, ça tombe à plat là-bas, ça part en fumée, et c'est resté ainsi, c'est compliqué». Comédien, Mittelsächsiches Theater, Freiberg, décembre 2001.

«Un exemple: nous avons joué Kabale und Liebe, ça a été la première pièce qu'on a jouée à Altenburg après la fusion [suite au partage des genres, les comédiens sont installés à Gera]. Et au premier rang, il y avait tous les membres de l'association des amis du théâtre d'Altenburg, et de façon démonstrative, ils n'ont pas applaudi. C'est quelque chose qui ne s'oublie pas. Et aujourd'hui encore, on sent des tensions ». Comédien, théâtre d'AltenburgGera, Gera, avril 2002.

Pour éviter que l'une des deux villes (et donc l'un des deux publics) ne se sente lésée par la fusion, les intendances tentent de jouer sur la répartition des premières et des représentations entre les différents lieux scéniques.

«Nous faisons vraiment des efforts désespérés depuis six ans, pour expliquer à Döbeln et à ses habitants qu'ils sont pour nous tout aussi importants [que Freiberg]. Nous essayons de faire quelques premières à Döbeln, nous appelons chacune des premières représentations premières. Et nous avons aussi essayé d'organiser des fêtes autour des premières dans le théâtre [...] mais les gens de Döbeln ne viennent pas. [...] Nous avons même conçu des affiches spéciales pour Döbeln [pour dissimuler l'asymétrie des représentations]». Chef dramaturge, Mittelsächsisches Theater, Freiberg, avril 2001.

Dans le cas de Plauen-Zwickau, l'alternance des premières est prévue par le contrat de fusion : « Dans les deux lieux scéniques, les mises en scène prévues par la programmation devront être réalisées. Les premières auront lieu alternativement à Zwickau et à Plauen. Cela vaut pour le Schauspiel comme pour le Musiktheater. Pour les concerts, des premières seront organisées en alternance. Ainsi, le public des premières doit se voir garantir, dans les deux villes, l'accès au spectre complet de la production $»(11)$. Pourtant, si l'on regarde par exemple les chiffres de la saison 2001-2002, on trouve sept premières à Plauen (dont une uniquement à Plauen) et cinq à Zwickau (dont deux uniquement à Zwickau). L'avantage donné par l'intendance à Plauen (qui craignait d'être le parent pauvre de la fusion), éveille le ressentiment du public de Zwickau, qui dénonce volontiers les faveurs accordées à Plauen.

En dehors des premières, la répartition des représentations présente souvent les mêmes déséquilibres. Ainsi dans le cas d'Altenburg et de Gera, le déséquilibre est assez marqué : entre 1995 et 2000 , on a en moyenne (toutes salles confondues) 776 représentations par an à Gera et seulement 456 à Altenburg. Toutefois, ici, l'écart observé est d'abord lié à la taille très différente des deux villes (qui se traduit en termes de public potentiel). Contrairement aux apparences, la fusion est d'ailleurs loin d'être défavorable à Altenburg puisqu'en 1994, avant la fusion, la ville n'accueillait que 300 représentations.

\section{Quel impact à moyen/long terme ?}

Enfin, au-delà des conséquences organisationnelles des fusions théâtrales qui participent à un mouvement plus large de rationalisation des institutions culturelles, on peut s'interroger sur l'hypothèse d'un effet retour de la réunification. Si le paysage théâtral des nouveaux Länder semble s'être progressivement adapté aux exigences structurelles de la réunification, les difficultés rencontrées - et qui sont souvent exacerbées dans le cas des fusions - ne conduisent-elles pas à fragiliser les principes mêmes du fédéralisme culturel ?

\section{Le cercle sans fin des restrictions}

La fusion est toujours présentée par les responsables de politiques culturelles des Länder comme un moyen de stabiliser les coûts de production tout en maintenant le niveau artistique (selon l'hypothèse qu'en l'absence de fusion les restrictions budgétaires imposées aux théâtres risquent de nuire au niveau moyen de leurs productions). La fusion est donc vue comme une forme de rationalisation des restrictions. Pour autant, la fusion se révèle toujours $a$ posteriori comme le point de départ et non pas comme l'aboutissement d'un processus de restriction.

Le cas d'Altenburg-Gera, une fusion qui intervient en 1995, est ici exemplaire. En effet, dans les années qui précèdent (1990-1994), les deux théâtres ont connu de fortes restrictions budgétaires, qui se sont traduites par des réductions de postes (de 285

(11) Grundlagenvertrag zwischen den Städten Zwickau und Plauen zur Fusion beider Theater, p. 2, version du 10 août 1998. 
à 224 à Altenburg et de 320 à 298 à Gera). Ces dernières touchent principalement l'administration et l'entretien, qui est privatisé. Mais entre 1995 et 1999, 79 autres postes ont été supprimés, faisant passer les effectifs du nouvel ensemble issu de la fusion de 534 à 455 . Et cette fois, ces réductions touchent aussi les professions artistiques. Puis pendant la saison 2000-2001, le budget du théâtre est réduit de 40 à 32 millions de $\mathrm{DM}$, ce qui entraîne brutalement cent nouvelles suppressions de postes et provoque la démission de l'intendant qui avait conduit la fusion. Les départements techniques et administratifs perdent alors 30 à $60 \%$ de leur personnel. Parmi les professions artistiques, quatorze postes sont supprimés pour le théâtre, deux pour le ballet et cinq pour l'opéra. Parallèlement, la nouvelle intendance diminue d'un tiers le nombre de représentations, puisque le manque de personnel technique rend désormais difficile la mise en place de représentations en parallèle dans les différents lieux scéniques.

\section{Les négociations de Haustarif}

Qui plus est, les restructurations postérieures aux fusions s'accompagnent fréquemment de l'adoption d'un Haustarif, une grille de salaire spécifique qui s'applique à un théâtre et par laquelle certaines catégories de personnels renoncent à des augmentations de salaire, voire même acceptent des rémunérations inférieures aux normes en vigueur dans les théâtres publics(12). Depuis 1994, pas moins de soixantedix accords de Haustarif (Haustarifverträge) ont été signés au niveau local, dont la grande majorité dans des théâtres des nouveaux Länder. Les entités fusionnées sont ici en première ligne.

Ainsi, au sein du Mittelsächsiches Theater, comme à Altenburg/Gera, un Haustarif a été mis en place après la fusion pour garantir (au moins provisoirement) le maintien des postes.

«L'accord d'établissement sur les salaires a pour origine le manque d'argent. Ceux qui nous financent, les pouvoirs publics, ne donnent pas plus d'argent que ce qu'ils donnaient auparavant, pas moins non plus ; mais les coûts, et en particulier les coûts sala-

(12) En Allemagne, il n'existe pas comme en France de salaire minimum fixé par l'État. Les normes salariales sont les résultats d'accords entre les partenaires sociaux. C'est le cas pour le théâtre public, qui dispose, au sein du NV Bühne (Normal Vertrag Bühne, le contrat qui encadre les activités artistiques dans les ensembles publics) d'un Tarifvertrag (accord salarial). Ce dernier est négocié par le Deutsche Bühnenverein (qui réunit les intendants et se situe donc du côté des employeurs) et par les principaux syndicats (la GDBA, mais aussi la Vereinigung Deutscher Opernchöre, qui représente les intérêts des choristes et la Gewerkschaft Deutscher Orchestervereinigung, qui regroupe les musiciens d'orchestre). Récemment révisé au 1er janvier 2003, le $N V$ Bühne fixe un salaire minimum mensuel de 1550 euros. riaux augmentent(13). Et pour sortir de cet écueil, l'ensemble a proposé de renoncer à une partie de son revenu dans les prochaines années, à $13 \%$, et en contrepartie il y a une sécurité de l'emploi. Les collègues ont renoncé à de l'argent, parce que nous avons tous pensé : si nous n'y renonçons pas, vingt ou vingt-cinq postes vont disparaître et si ce théâtre voit ses postes diminuer, cela va forcément jouer sur son potentiel artistique, et un mauvais théâtre est plus facile à éliminer de la carte qu'un théâtre qui a une bonne réputation dans la Saxe. C'est pour cela que les collègues ont pris cette décision, il y a eu un vote et $80 \%$ étaient pour. [...] Mais les salaires ici sont très bas et renoncer à $13 \%$ a été douloureux. Mais d'un autre côté, nous avons ainsi acheté le maintien de postes pour quelques années». Chef dramaturge, Mittelsächsiches Theater, Freiberg, avril 2001.

Si son organisation a entériné localement nombre de ces accords, en partenariat avec les syndicats et les municipalités de tutelle des théâtres concernés, le directeur général du Deutsche Bühnenverein, Rolf Bolwin, n'en souligne pas moins le danger à moyen terme de ces grilles tarifaires spécifiques: «Ces grilles salariales maison se retrouvent pour l'essentiel dans les nouveaux Länder. [...] Elles ont été pensées comme des solutions transitoires. [...] Mais on a constaté que cela ne suffirait pas et que la durée envisagée était trop courte. Pourtant, le fait que les théâtres des nouveaux Länder sortent du système de relations professionnelles en vigueur n'est certainement pas une solution. Les théâtres ont besoin de règles précises du côté de l'employé, pour pouvoir travailler sans conflit. De plus, il ne doit pas y avoir dans ces théâtres et ces orchestres des grilles salariales qui, sur la durée, seraient plus faibles que dans les anciens Bundesländer, c'est-à-dire qu'il ne doit pas y avoir de zone tarifaire de l'Est». Rolf Bolwin, mdr online, 27 août 2001.

\section{L'effet retour de la réunification?}

Les craintes exprimées en 2001 par Rolf Bolwin semblent bien s'être concrétisées et apparaissent même relativement modestes au vu des évolutions observées depuis le début de la décennie. En effet, non seulement les théâtres de l'Est ont adopté durablement des grilles tarifaires inférieures aux normes en vigueur au sein de la République fédérale, mais on observe clairement une extension des cas de Haustarifverträge qui va des petits aux grands théâtres et surtout des nouveaux aux anciens Länder. Le mouvement qui s'est amorcé à l'Est a donc fait tâche d'huile. Après avoir touché les scènes les plus prestigieuses des nouveaux Länder (notamment le

(13) Depuis 1990, les salaires de l'Est rattrapent progressivement les salaires de l'Ouest, ce qui entraîne de fait une augmentation de la masse salariale dans les théâtres. Les pouvoirs publics prônent donc des réductions d'effectifs pour contrer cette hausse. 


\section{Encadré 4}

\section{Un usage préventif du Haustarif qui fait débat : le Weimarer Modell (modèle de Weimar)}

Les projets de fusion lancés par le Land de Thuringe afin de restructurer son paysage théâtral n'épargnent pas la capitale du Land (Erfurt, 213000 habitants) et sa prestigieuse voisine (Weimar, 62000 habitants), distantes de $25 \mathrm{~km}$. On assiste durant l'année 2001 à une véritable lutte d'influence entre les deux villes. La municipalité et les habitants de Weimar, qui occupe une position unique dans l'histoire et dans l'imaginaire culturel allemand, s'opposent farouchement à l'idée d'un rapprochement avec Erfurt et d'une séparation des genres. Le Deutsche Bühnenverein publie une étude de faisabilité qui évalue positivement les retombées potentielles de la fusion (1) et recommande la création d'une "Theatergemeinschaft» (communauté de théâtres) associant, sous une forme de $\mathrm{GmbH}$, trois tutelles publiques (les villes d'Erfurt et de Weimar ainsi que le Land de Thuringe). Mais les initiatives citoyennes se multiplient et le conseil municipal de Weimar rejette définitivement le projet du Land en février 2002. L'intendant du Deutsches Nationaltheater (DNT) Weimar, Stefan Märki, est chargé par la ville d'élaborer des propositions alternatives. Avec l'aide d'un avocat berlinois, Peter Rau, il propose une forme particulièrement radicale de Haustarif.

Si les arguments avancés sont classiques (faire face au poids croissant des salaires dans le budget du théâtre et tenter de maintenir le nombre de postes - et donc la qualité artistique de l'Ensemble malgré la baisse des subventions publiques), l'initiative de S. Märki se distingue des autres cas évoqués dans cet article. En effet, elle ne s'inscrit pas dans le cercle vicieux de restrictions provoqué par une fusion (qui n'a pas lieu), mais intervient en amont pour tenter de proposer une alternative à la restructuration du paysage théâtral. C'est sans doute cette singularité, couplée au prestige de l'Ensemble concerné, qui confère au cas de Weimar sa position spécifique dans le débat sur l'avenir du système théâtral des nouveaux Länder, et plus largement de l'Allemagne réunifiée.

De manière assez classique, les membres de l'Ensemble renoncent à leur 13e mois et à toute augmentation jusqu'en 2008. En contrepartie, l'intendance s'engage à bloquer les licenciements et à lutter sans relâche contre toute tentative de fusion. Mais plus qu'un ajustement temporaire, le modèle de Weimar se veut « un premier pas sur le chemin conduisant à de nouvelles structures pour les théâtres municipaux allemands» (2). En changeant de statut juridique pour devenir une $\mathrm{GmbH}$ à laquelle la municipalité n'est pas associée (3), le DNT impose à ses membres de nouveaux contrats de travail (4) et pose directement la question d'une « privatisation » de la culture. En effet, ce faisant le théâtre n'est plus soumis aux grilles tarifaires en vigueur dans le service public allemand (pour ce qui concerne les professions non artistiques) ni aux conventions négociées par les syndicats et le Bühnenverein (en ce qui concerne les professions artistiques).

Les débats suscités par ce changement de statut tendent à cristalliser les positions des différents acteurs sociaux concernés. Du point de vue de l'intendant et de l'avocat P. Rau qui a activement participé à la conception du modèle, il s'agit de sortir d'une logique de fusions qui ne fait que « cimenter les problèmes structurels existants et qui s'avère contreproductive pour le théâtre » (5). Pour cela, il faut selon eux autonomiser les théâtres et les dégager des pièges d'une organisation tarifaire contraignante et peu adaptée aux exigences concrètes du travail théâtral. Face à ces discours qui remettent en cause les fondements mêmes du droit du travail allemand (et pas seulement du droit du travail théâtral), les différents syndicats sont plus ou moins virulents. Ver.di, qui représente les professions non artistiques du théâtre (6), dénonce une américanisation de la culture et une « fuite » en dehors de toute règlementation qui pourrait s'étendre à d'autres secteurs d'activité. La GDBA, principal syndicat réunissant les professions artistiques du théâtre, est plus modérée et reproche à ver. di d'aiguiser les tensions entre catégories de personnel au sein du théâtre, sans prendre la mesure de la crise qui menace le DNT. Hostile à la généralisation du modèle de Weimar, la GDBA craint en effet avant tout la fermeture du théâtre et a déjà entériné, quand elles semblaient inévitables, des adaptations locales, temporaires et négociées sous forme de Haustarife. Enfin le Bühnenverein, considéré comme un « dinosaure » par S. Märki qui ne lui pardonne pas d'avoir soutenu le projet de fusion avec Erfurt, tente d'apaiser les tensions et ce d'autant plus que l'affaire de Weimar intervient quelques mois à peine avant l'entrée en vigueur, au 1er janvier 2003, du nouveau contrat de soliste (Normal Vertrag Bühne) qui représente pour le Bühnenverein l'aboutissement de 10 ans d'effort et de négociations avec les syndicats représentant les professions artistiques. Rolf Bolwin prend ainsi le parti de neutraliser l'initiative de S. Märki en la resituant dans le contexte des nombreux Haustarife signés dans d'autres théâtres, notamment est-allemands, avec l'accord de son organisation : « En pratique, ce soi-disant modèle repose en fait sur une série de mesures d'économies budgétaires déjà proposées ailleurs, comme par exemple le renoncement aux congés payés et au 13e mois, que nous avons souvent mis en place avec l'accord des syndicats dans des théâtres et des orchestres des nouveaux Länder»(7). Mais dans les faits, la rupture est bien plus radicale et le DNT, en se détachant des autorités publiques et en renonçant à appartenir au Bühnenverein, affirme clairement sa position singulière au sein du paysage théâtral allemand.

(1) Machbarkeitsstudie zur Umsetzung der Empfehlungen der Sachverständigenkommission "Theater und Orchester in Thuringen" (EverdingKommission) aus dem Jahre 1996 - Die Theatergemeinschaft des Theaters Erfurt und des Deutschen Nationaltheaters Weimar", Deutsche Bühnenverein, Cologne, 2001.

(2) Site Internet du DNT, septembre 2006. On peut noter que cette formulation témoigne d'une évolution nette des positions de l'intendant S. Märki qui, en 2002/2003 au moment de la mise en œuvre du changement de statut de son institution, refusait le terme de «modèle» et insistait avant tout sur les conditions sociales, politiques et historiques propres à la ville de Weimar. Depuis, la médiatisation de la stratégie adoptée par le DNT et l'aggravation des problèmes financiers rencontrés par les théâtres allemands, dans les anciens comme dans les nouveaux Länder, ont donné un poids beaucoup plus fort à son initiative, qui est par exemple largement discutée au sein des différents partis politiques.

3) Notons qu'au début des années 1000 , environ $25 \%$ des théâtres publics allemands sont des GmbH. Mais ils continuent à être gérés par des tutelles publiques. Ce n'est plus le cas du DNT, qui devient une GmbH gérée par des personnes privées. Le seul antécédent était le cas du Berliner Ensemble, dont le dossier avait déjà été suivi par l'avocat P. Rau. La municipalité de Weimar, qui soutient ce changement de statut, continue néanmoins à subventionner le DNT et s'engage même à maintenir le niveau de ses subventions jusqu'en 2008.

(4) Dans les faits, les contrats antérieurs restent valables pendant un an. Passé ce délai, de nouveaux contrats sont signés au nom de la GmbH, que les salariés de l'Ensemble ont la possibilité de refuser. Théoriquement, ils restent alors employés par la ville. Mais cette dernière peut les licencier si elle prouve qu'elle n'est pas à même de leur fournir un autre emploi correspondant à leurs compétences. La ville de Weimar ne comptant qu'un seul théâtre, cette clause est extrêmement contraignante pour les membres de l'Ensemble qui seraient tentés de refuser le contrat que leur proposera la nouvelle $\mathrm{GmbH}$.

(5) S. Märki in Neue Musikzeitung, 2002/2003, p. 3.

(6) Relevant du service public du service public (au contraire des professions artistiques qui dépendent d'un contrat spécifique, le Normal Vertrag Bühne), ce sont elles qui ont le plus à perdre dans le changement de forme juridique de leur institution.

(7) R. Bolwin, in Theaterheute, août 2002, p. 1. 
Semperoper de Dresde), il s'étend désormais dans les anciens Länder où les théâtres du SchleswigHolstein et de Saarbrück sont aujourd'hui en première ligne.

De manière intéressante, et toutes choses égales par ailleurs, nous retrouvons ici une évolution mise en évidence dans d'autres domaines d'activité. Olivier Giraud et Michel LaLlEment (2001) soulignent ainsi qu'une reconfiguration des relations professionnelles est en cours dans les nouveaux Länder, qui répond à un mouvement conjoint «de décentralisation et de différentiation des conventions collectives». La réunification s'est accompagnée, dans un premier temps, de la signature d'accords concernant le rattrapage des salaires de l'Est sur ceux de l'Ouest. Mais dans le monde des théâtres publics, comme ailleurs, le compromis s'est avéré difficilement tenable. Les difficultés rencontrées se sont traduites «par l'activation de solidarités d'entreprise, les objectifs prioritaires étant la survie de l'unité productrice et l'adaptation aux nouvelles donnes du marché, quitte à promouvoir [...] des conditions de travail difficiles» (GIRAuD, LALLEMENT, 1998). Les accords locaux signés à l'Est, dès lors qu'ils ont essaimé dans les anciens Länder, ont fini par irriter les Allemands de l'Ouest, qui ont reproché à leurs concitoyens de casser les accords de branche pour sauver localement des emplois.

Les positions des principaux acteurs ont alors sensiblement évolué. Ainsi la GDBA, pourtant signataire des accords de Haustarif au sein des théâtres de l'Est, s'inquiète désormais de ce qui devient progressivement une règle, et non plus une exception destinée à accompagner la période de transition à l'Est. Lors de son assemblée générale de 2005, elle interpelle ainsi le Bühnenverein et demande aux intendants de veiller, au sein de leurs théâtres respectifs, au maintien du Tarifvertrag et au respect des conditions du Normal Vertrag Bühne. Rappelant que le monde théâtral «n'est pas une zone de non droit », la GDBA souligne avec force le rôle structurant du Tarifvertrag comme « instrument de régulation des conditions de travail ». Un éditorial de la même année dénonce l'érosion généralisée du paysage théâtral allemand et l'irréversibilité des accords signés localement (HerdLeIn, 2005).

Au niveau de la branche, le syndicat majoritaire des artistes de théâtre opère donc un rappel à la règle et aux conventions régissant le monde du travail théâtral. Mais au cas par cas, ses représentants se retrouvent toujours aux côtés du Bühnenverein pour sauvegarder les emplois menacés, quitte à accepter des conditions de salaire moins avantageuses. Ils se heurtent, au sein des institutions théâtrales, au syndicat ver.di. (14) (Vereinte Dienstleistungsgewer $k s c h a f t$ ) qui représente notamment les intérêts des personnels techniques des théâtres et qui s'oppose farouchement à la mise en place des Haustarife. Les institutions théâtrales se trouvent donc de plus en plus fréquemment confrontées à des conflits entre personnels artistiques (encore majoritairement favorables aux Haustarife lorsqu'ils permettent de sauvegarder des emplois) et personnels techniques (hostiles à ces accords(15)).

De ce point de vue, l'intégration des nouveaux Länder au sein de la République fédérale semble bien conduire progressivement à la remise en cause des principes fondamentaux sur lesquels s'étaient construites les formes de régulation du travail en RFA (BAFOIL, 1996).

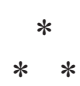

Le processus des fusions théâtrales qui s'est enclenché en Saxe et en Thuringe dans la première moitié des années 1990 ne semble pas encore être arrivé à son terme aujourd'hui. De nouvelles fusions se dessinent, qui impliquent des entités théâtrales déjà fusionnées, et qui parfois remettent en cause les fusions antérieures, témoignant ainsi de la complexité du processus de recomposition du paysage théâtral au sein des nouveaux Länder.

En effet, l'impact des fusions sur la stabilisation des coûts de fonctionnement des structures théâtrales publiques est difficile à évaluer. L'efficacité de cette stratégie de concentration du tissu théâtral reste donc incertaine, et ce d'autant plus que l'on ne dispose pas de statistiques fiables. La réticence des intendances et des ministères de la Culture à communiquer sur ces questions montre que les effets positifs espérés à moyen et long terme sont loin d'être évidents. Les enjeux des fusions au sein même des organisations théâtrales, leurs conséquences sur le travail au sein des ensembles, sont plus faciles à cerner. Ils mettent en évidence la force des interdépendances entre les politiques culturelles menées à l'échelle des Länder et les transformations organisationnelles qui affectent localement les structures concernées. Plus encore, les difficultés observées révèlent la fragilité du modèle théâtral hérité de la RDA et ses difficultés à affronter le changement de système. En ce sens, les fusions apparaissent

(14) Fondée en 2001 à Berlin, cette organisation est née du rapprochement de cinq syndicats présents dans le secteur des services. Elle a fait des politiques tarifaires son principal cheval de bataille.

(15) Rappelons que depuis la réunification, les départements techniques des théâtres, et c'est particulièrement vrai dans les cas de fusion, ont été les premiers touchés par les réductions de poste, qui sont intervenues plus tardivement et surtout moins massivement pour les professions artistiques. Les sacrifices déjà consentis localement par ces deux catégories au sein des ensembles sont donc très différents. 
bien pour le sociologue comme des cas extrêmes, qui cristallisent les problèmes rencontrés de façon générale par l'ensemble des théâtres publics des nouveaux Länder (VERDALLE (DE), 2003, 2006).

Mais dans le même temps, ces fusions remettent aussi en cause un modèle ouest-allemand qui sort fragilisé de son application à un tissu théâtral dont les caractéristiques étaient très éloignées de celles des anciens Länder. Loin d'exercer une influence à sens unique, la réunification pourrait donc bien conduire à une altération durable des principes fondateurs du fédéralisme ouest-allemand (Le GlOANNEC, 2001).

\section{Bibliographie}

BAFOIL F. (1996), « La crise des relations professionnelles en Allemagne de l'Est et l'évolution de la négociation paritaire allemande », in Politix, $\mathrm{n}^{\circ} 33$, pp. 87-110.

BARMEYER C. (2005), « Rapprochements des entreprises et rapprochements des hommes. Regard interculturel sur le leadership dans les coopérations franco-allemandes "), in Joly H. (sous la direction de), (2005), Formation des élites en France et en Allemagne, travaux et documents de CIRAC, Paris.

Giraud O., Lallement M., (1998), « Construction et épuisement du modèle néocorporatiste allemand. La réunification comme consécration d'un processus de fragmentation sociale », in Revue Française de Sociologie, n 43-1, pp. 39-69.

Giraud O., Lallement M., (2001), « L'État et les relations professionnelles en Allemagne: interactions et recompositions après la réunification ", in Le Gloannec A.-M. (sous la direction de), (2001), L'État en Allemagne. La République fédérale après la réunification, Presses de Sciences Po, Paris.

Herdlein H., (1993), « Einigungsvertrag verpflichtet kulturelle Substanz erhalten! », in Bühnengenosseschaft, $\mathrm{n}^{\circ} 5$, pp. 5-6.

Herdlein H. (2005), «Kulturelle Substanz erhalten - Arbeitslosigkeit im Entstehen bekämpfen », in Bühnengenosseschaft, ${ }^{\circ}$ 5, pp. 4-5.
Laborier P., (1992), «L'enjeu de la culture dans l'unification allemande. Du dirigisme centraliste au pluralisme démocratique ", in Allemagne d'aujourd'hui, n 121, pp. 146-165.

LABORIER P., (1996), "Conservation ou rénovation? Transitions de la politique culturelle », in Politix, $\mathrm{n}^{\circ} 33$, pp. 111-132.

Le Gloannec A.-M (sous la direction de), (2001), L'État en Allemagne. La République fédérale après la réunification, Presses de Sciences Po, Paris.

Verdalle L. (de) (sous la direction de), (2002), « D’une gestion centrale à une gestion locale de la culture : les théâtres au cœur de la transition est-allemand », in Nouvelle Alternative, $\mathrm{n}^{\circ}$ 57, pp. 12-22.

Verdalle L. (de), (2003), « La transition théâtrale dans l'ex-RDA: vers la recherche de nouvelles formes de régulation organisationnelle », in Sociologie du travail, vol. 45 , pp. 385-405.

Verdalle L. (de), (2006), Le théâtre en transition. De la RDA aux nouveaux Länder, collection « Dialogiques », éditions de la MSH, Paris.

WiesAnd A., (1992), « Vers le centralisme et la culture d'État? Sur le financement de la culture en Allemagne », Le Débat, $\mathrm{n}^{\circ} 70$, pp. 26-41. 


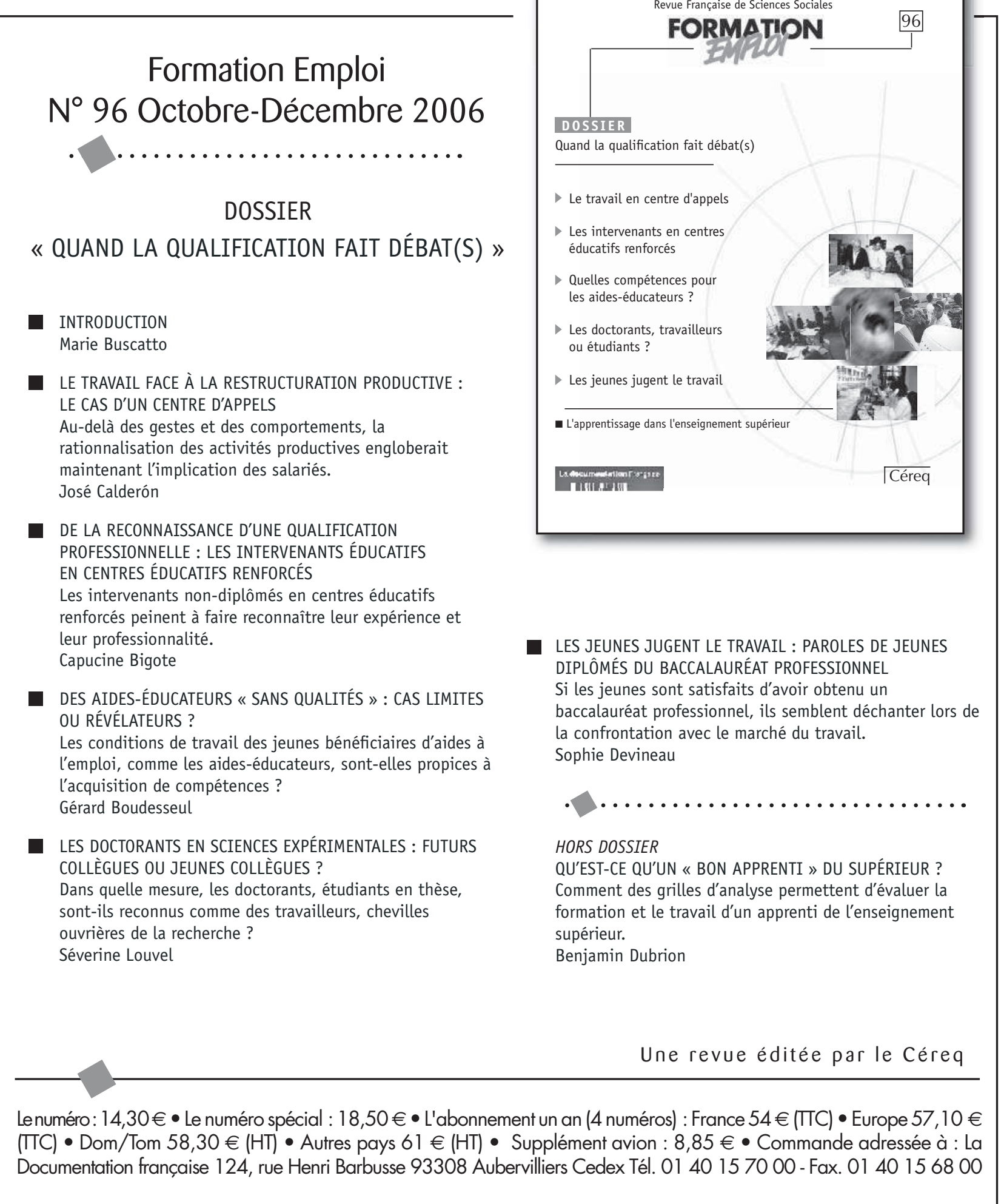




\title{
La relation innovation emploi dans les services
}

\author{
Faridah Djellal, Faïz Gallouj ( ${ }^{*}$
}

La question de l'innovation dans les services et de ses relations à l'emploi est une question fondamentale, qui paradoxalement n'occupe pas la place qui lui revient dans la littérature économique. Cet article tente, à titre essentiellement programmatique, d'articuler la question de l'emploi aux principaux travaux empiriques ou théoriques qui ont été consacrés à l'innovation dans les services. Bien qu'unanimement considérée comme fondamentale, cette question est relativement peu exploitée: en effet, l'une des variables de la relation (à savoir la question de l'innovation dans les services) n'est pas encore clairement élucidée sur le plan théorique. Le caractère encore flou de la nature et du statut de l'innovation dans les services conduit à considérer comme hasardeuse toute tentative d'analyse des conséquences de cette innovation sur l'emploi. Il s'agit-là pourtant d'un champ de recherche important sur le plan théorique et des politiques publiques qu'il faudrait explorer davantage, à la fois sur le plan micro, méso et macroéconomique, que ce soit par le biais de la construction théorique déductive ou de l'analyse inductive qualitative ou statistique. Ce sont les deux axes généraux de recherche que l'article explore, dans une volonté de fournir un premier cadrage pour une réflexion à développer.

La question de la relation entre le changement technique et l'emploi est une question ancienne et fondamentalement complexe, à la fois sur le plan théorique et empirique, indépendamment même du problème du secteur concerné (FreEman, Soete, 1987; Petit, 1995; Vivarelli, 1995). Elle renvoie à des causalités multiples et contradictoires, directes et indirectes. Elle ne semble pas pouvoir être abordée de manière satisfaisante par l'intermédiaire d'un nombre réduit de mécanismes généraux, ni à un seul niveau d'analyse, micro, méso ou macroéconomique. Les débats autour de la «théorie de la compensation» (selon laquelle les ressorts du marché sont en mesure de compenser, de manière automatique, les pertes d'emplois induites par une innovation destructrice d'emplois(1)) donnent une idée de la complexité des mécanismes à l'œuvre. L'écheveau des relations est par ailleurs rendu encore plus inextricable par le fait que d'autres variables que l'innovation interviennent sur la croissance de l'emploi dans les services: la demande, les changements institutionnels, etc. Il n'est donc pas étonnant, comme le soulignent Vivarelli et Pianta (2000) que l'économie néoschumpeterienne contemporaine de l'innovation (à l'exception notable des travaux de Freeman, Clark, Soete, 1982; Freeman,

\footnotetext{
*Clersé, Ifrési et université de Lille 1; faiz.gallouj@univ-lille1. fr; faridah.djellal@univ-lille1.fr

(1) On considère généralement que la compensation opère à travers les différents mécanismes suivants: la création de nouvelles machines, la baisse des prix, de nouveaux investissements, la baisse des salaires, l'augmentation des revenus, la création de nouveaux produits (Vivarelli, 1995 ; Petit, 1995).
}

SoEte, 1987) ait évité cette question. Il l'est encore moins qu'elle soit généralement absente des travaux consacrés à l'innovation dans un secteur qui pose de nombreux autres problèmes théoriques intéressants et difficiles (en particulier ceux de la définition, de la mesure, des modes d'organisation et d'appropriation de l'innovation et de la R-D).

Ainsi, bien que le secteur des services soit reconnu comme le principal créateur d'emplois dans les économies contemporaines, trop peu de travaux se sont penchés sur la question du lien entre l'innovation dans ce secteur et l'emploi. Ce désintérêt pour une question théorique importante et ancienne peut être interprété de différentes manières. Tout d'abord, dans la mesure où la littérature économique a longtemps sous-estimé l'innovation dans les services, il n'est pas étonnant qu'elle ait également sous-estimé et négligé ses effets sur l'emploi. Ensuite, la loi d'Engel et la loi de la productivité ont fourni des arguments théoriques satisfaisants pour expliquer la croissance des services et de l'emploi dans les services (Fourastié, 1949; BAUMOL, 1967; Bell, 1976). En effet, selon la première de ces lois, la demande et la consommation de services augmentent dans des économies dont les revenus sont croissants, et selon la seconde, le faible taux de croissance de la productivité dans les services, y entraîne un accroissement de l'emploi. Les principales théories de la croissance tertiaire ont ainsi écarté l'argument de l'innovation dans les services (ou se sont contentées du constat de la faiblesse de l'innovation dans ce secteur), pour expliquer le glissement inéluctable d'une économie industrielle à une économie de services. Enfin, les travaux, qui, 
en particulier aux États-Unis, se sont efforcés de dénoncer la mauvaise qualité des emplois engendrés par l'économie des services ont également contribué, à leur manière, à occulter la question du lien innovation-emploi. En effet, sous l'angle de la création d'emplois, la société de service ne serait rien d'autre qu'une "société de serviteurs», une hamburger society, une bad jobs society, essentiellement génératrice de «McJobs» (Bluestone, HARRISON, 1986; Thurow, 1989; MaHAR, 1992). Il est peu probable qu'une telle société soit propice à l'innovation.

Notre objectif, dans cet article, est de proposer une relecture, à la lumière de la question de l'emploi, des travaux consacrés à l'innovation dans les services. Ces travaux sont souvent divisés en deux groupes distincts (GallouJ, 1994). Le premier groupe (les approches technologistes) est constitué de travaux qui réduisent, pour l'essentiel, l'innovation dans les services à l'adoption de systèmes techniques, tandis que le second groupe (les approches servicielles), réunit les travaux qui mettent l'accent, en particulier, sur les formes non technologiques de l'innovation.

Cet article se propose d'évaluer dans quelle mesure et sous quelle forme la question de l'emploi est implicitement ou explicitement présente ou mérite d'être introduite dans les deux groupes ainsi identifiés. Il s'agit donc de rendre compte, que ce soit au niveau de la firme, du secteur ou au niveau macro-économique, de la façon dont la question de l'emploi s'articule (ou peut s'articuler) avec celle de l'innovation, lorsqu'il s'agit des services. Ce projet constitue à la fois un bilan de la littérature et un agenda de recherche. À ce titre, il pose beaucoup plus de questions qu'il n'apporte de réponses.

\section{La question de l'emploi au cœur des approches technologistes}

Les travaux identifiant l'innovation dans les services à l'innovation technologique (adoptée par les services) sont, de loin, les plus anciens et les plus nombreux, ce qui contribue, dans une certaine mesure, à sous-estimer d'autres aspects de l'innovation. Au-delà des interprétations théoriques (notamment les hypothèses associées au concept de fonction de production, qui accorde une place centrale aux innovations de processus), le principal argument qui peut justifier une telle orientation des travaux de recherche est que les services sont des secteurs de plus en plus intensifs en technologies et en capital.

On peut décomposer cet axe technologiste en trois groupes de travaux, différents par leur degré d'ambition théorique, le type d'innovation technologique qu'ils privilégient, et disproportionnés quant à leur taille. Le premier groupe est consacré aux consé- quences de l'introduction de systèmes techniques dans les firmes ou secteurs de services (analyses en termes d'impacts). Nous distinguerons ici les analyses en termes d'impacts des TIC des analyses plus générales en termes d'impacts de l'innovation technologique quelle qu'elle soit. Le second groupe ne comporte qu'une seule référence (le modèle du cycle de vie inversé de Barras), qui a pour ambition de proposer une théorie de l'innovation dans les services. Le dernier groupe est constitué de travaux d'inspiration évolutionniste, qui visent à retracer des trajectoires d'innovation dans les services. Seuls les deux premiers groupes envisagent explicitement la question de l'emploi. Bien que les approches taxonomiques initiales (celles de Pavitt ou de Soete et Miozzo) n'abordent pas explicitement cette question, on peut considérer qu'elle y est implicitement présente ou qu'elle peut y être introduite, dans une certaine mesure, à travers les implications en termes d'emplois des formes d'innovation et de la nature des trajectoires envisagées.

\section{L'impact des technologies informationnelles sur l'emploi dans les services}

Une part considérable de la littérature consacrée à l'innovation dans les services s'intéresse aux conséquences de la diffusion des technologies informationnelles aux niveaux micro, méso et macro-économiques (Bertrand, Noyelle, 1987; Cossalter, HÉZARD, 1983; DJellal, 2002). Pour simplifier, on peut décrire l'expansion de ces technologies à travers deux modèles d'innovation: l'informatique lourde, d'une part, et l'informatique décentralisée et les réseaux, d'autre part. Une manière commode de rendre compte sinon des résultats du moins des principaux questionnements généraux envisagés dans cette abondante littérature (en termes d'impacts) est de croiser ces deux modèles avec un ensemble de variables économiques fondamentales comme l'emploi, les qualifications et l'organisation des tâches, la productivité, le caractère échangeable $\mathrm{du}$ «service » et sa qualité ( $c f$. figure 1).

Figure 1

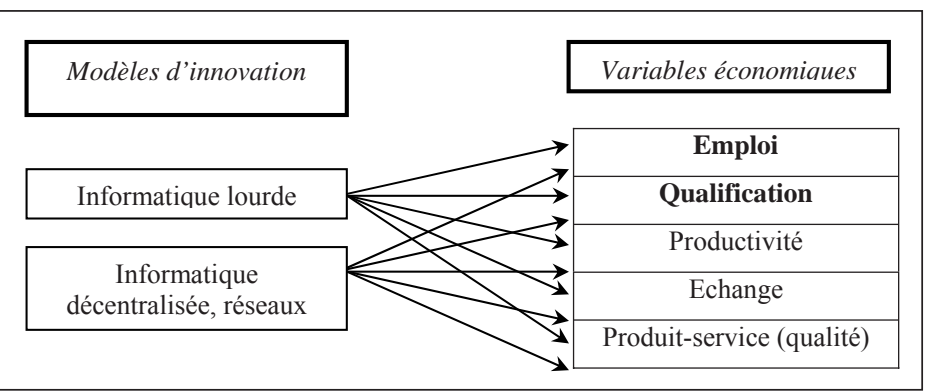

Les analyses en termes d'impacts des TIC

Le modèle de l'informatique lourde aurait ainsi plutôt tendance à exercer un effet positif sur la productivité et l'échange, mais négatif sur l'emploi 
et la qualification de la main-d'œuvre. Ce modèle d'innovation correspond à l'informatisation des back-office (c'est-à-dire des activités qui ne se trouvent pas au contact des clients) et vise avant tout à la réduction du coût de la prestation, par la standardisation des tâches et l'exploitation d'économies d'échelles. Quant au modèle de l'informatique décentralisée et des réseaux, il aurait plutôt tendance à exercer des effets positifs sur l'emploi (ou sur certains types d'emplois), la qualification, la facilité d'échange, mais aussi éventuellement sur la productivité et la qualité du "produit». Ce second modèle modifie le front-office (c'est-à-dire l'interface avec les clients). Il engendre des économies de variété et réduit les tâches routinières en faveur d'activités commerciales et de conseil.

Les réflexions précédentes ne constituent pas des réponses ou des résultats définitifs, mais plutôt des hypothèses. Quel que soit le modèle d'innovation considéré, une partie importante de la littérature consiste à présenter l'une, plusieurs ou l'ensemble de ces hypothèses théoriques et leurs mécanismes, à les confronter à la réalité et à tenter d'interpréter les écarts éventuels.

Quel que soit le modèle considéré, la question de l'innovation dans les services peut être abordée sous l'angle de ses conséquences sur d'autres dimensions qualitatives de l'emploi. On peut se poser la question de ses conséquences sur la distribution sexuelle de l'emploi. Un certain nombre de travaux (APPELBAUM, 1987; WeBSTER, 1996) suggèrent qu'en ce qui concerne l'emploi, l'innovation n'est pas sexuellement neutre, autrement dit qu'elle est biaisée selon le genre. ApPelBaum (1987) étudie la manière dont l'innovation modifie la structure de l'emploi dans le secteur de l'assurance de sorte que des fonctions qui étaient auparavant masculines se féminisent ( $c f$. aussi WeBster, 1986).

D'autres questions intéressantes sont, à notre connaissance, peu présentes dans la littérature. Il s'agit en particulier des conséquences de l'innovation dans les services sur ce que DevetTer (2001) appelle la disponibilité temporelle au travail, qu'on pourrait élargir à la disponibilité spatio-temporelle au travail. Certaines formes d'innovation technologique ou organisationnelle dans les services facilitent ou induisent des formes de travail à temps partiel, de dilution du temps de travail dans le temps domestique, ou des formes de mobilisation $\mathrm{du}$ travailleur dans des espaces spatio-temporels atypiques: le domicile dans le cas du télétravail, de l'usage de la téléphonie mobile, de l'ordinateur portable et de l'Internet.

C'est probablement dans ce groupe de travaux que la question de la relation innovation qualification est le plus souvent abordée. Il existe néanmoins une littérature beaucoup plus abondante centrée sur la thématique de la qualification des emplois tertiaires. Nous n'en rendons pas compte ici dans la mesure où la question de l'innovation en est absente ou n'y occupe qu'une place incidente. Nous avons d'ores et déjà cité en introduction un certain nombre de travaux essentiellement américains sur ce thème. Mais il existe également de nombreux travaux français (BERTRAND, 1988; JANY-CATRICE, 2004; GADREY, 2005). Bertrand propose ainsi une typologie des emplois de service qui distingue, sur la base de critères qualitatifs relatifs à l'emploi, les trois groupes suivants : 1) le tertiaire technico-administratif et socio-culturel; 2) les services commerciaux et personnels; 3) le tertiaire "para-industriel».

Pour saisir dans toute leur complexité les relations entre NTIC, services et emplois, on ne peut pas se contenter d'une analyse des NTIC comme facteur exogène (analyses en termes d'impacts). On assiste en effet à une endogénéisation croissante des NTIC dans l'économie des services. Les services ne sont plus ainsi simplement envisagés à travers leurs comportements d'adoption de ces NTIC. Ils peuvent jouer un rôle de plus en plus actif dans leur production et leur diffusion; et l'innovation de service apparaît souvent comme une catégorie hybride associant des NTIC et une activité d'ingénierie organisationnelle, c'est-à-dire de conception-développement de formules organisationnelles (Duellal et alii, 2003). Cette endogénéisation des NTIC est à l'origine de nouveaux produits-services, de nouvelles demandes, de nouvelles activités et de nouveaux marchés, et par conséquent de nouveaux emplois (Petit, Soete, 2001).

\section{L'impact de l'innovation technologique (sous toutes ses formes) sur l'emploi dans les services}

Tirant parti des enquêtes communautaires sur l'innovation (ECI), un certain nombre de travaux statistiques récents et en nombre restreint (EvANGELISTA, 2000a; Evangelista, Savona, 2003; Nählinder et Hommen, 2002; Peters, 2004) se sont efforcés de mesurer les effets de l'innovation sur l'emploi et les qualifications dans les services ( $c f$. tableau 1).

La version italienne de 1'ECI 2, réalisée en 1997 pour la période 1993-1995, a ainsi collecté des données sur les impacts de l'innovation sur l'emploi total des entreprises de manière globale (la question posée étant: l'introduction de l'innovation a-t-elle conduit 1) à un accroissement de l'emploi; 2) à une baisse de l'emploi; 3) à aucun impact sur l'emploi?), puis, en dissociant trois niveaux de qualification «élevé, moyen, faible». Les questions et les données correspondantes ne sont pas associées à chacun des types d'innovation pris en compte par le questionnaire (à savoir: la création d'un nouveau service, l'amélioration d'un service existant, l'introduction d'un nouveau process). Ainsi, les réponses obtenues sont des «résultats» nets (de la somme des différents effets). En exploitant cette enquête, 
Tableau 1

\section{L'impact de l'innovation technologique sur l'emploi dans les services}

\begin{tabular}{|c|c|c|}
\hline Enquête & Description du dispositif & Principales conclusions \\
\hline $\begin{array}{l}\text { Italienne } \\
\text { EVANGELISTA } \\
(2000 \mathrm{a})\end{array}$ & $\begin{array}{l}\text { ECI } 2 \text { en } 1997 \text { période 1993-1995 } 6000 \\
\text { entreprises }\end{array}$ & $\begin{array}{l}\text { - Innovation : effet positif sur emploi } \\
\text { - Effet positif très fort pour les emplois très qualifiés } \\
\text { - Effet négatif pour les emplois les moins qualifiés } \\
\text { - Impact variable selon la taille des firmes : } \\
+ \text { grandes entreprises : effet destructeur d'emplois (surtout peu qualifiés) } \\
+ \text { petites entreprises : effet créateur }\end{array}$ \\
\hline $\begin{array}{l}\text { Suédoise } \\
\text { NÄHLINDER } \\
\text { et HOMMEN } \\
(2002)\end{array}$ & $\begin{array}{l}\text { ECI } 2 \text { période } 1994-1996 \\
743 \text { entreprises (dont } 140 \text { firmes de SIC) } \\
\text { L'analyse ne porte que sur les SIC }\end{array}$ & $\begin{array}{l}\text { - Innovation : effet positif sur l'emploi } \\
\text { - Effet positif sur les qualifications }\end{array}$ \\
\hline $\begin{array}{l}\text { Allemande } \\
\text { PETERS } \\
(2004)\end{array}$ & $\begin{array}{l}\text { ECI } 3 \\
\text { réalisée en } 2001 \\
\text { période } 1998-2000 \\
2200 \text { firmes industrielles et de services }\end{array}$ & $\begin{array}{l}\text { - Innovation de produit : impact positif sur l'emploi dans les services } \\
\text { - Innovation de process : pas eu d'effet négatif sur l'emploi dans les } \\
\text { services }\end{array}$ \\
\hline
\end{tabular}

Evangelista (2000a) constate ainsi que l'innovation exerce un effet globalement positif sur l'emploi dans les services. Cet effet positif intervient de manière particulièrement forte sur les emplois très qualifiés, tandis que les effets «destructeurs d'emplois» du changement technique jouent essentiellement sur les composantes les moins qualifiées de l'emploi. L'enquête montre également que l'impact de l'innovation varie selon la taille des firmes. Dans les grandes firmes, l'innovation exerce un effet «destructeur d'emplois» (qui affecte essentiellement les emplois peu qualifiés), tandis que, dans les petites firmes, son effet sur l'emploi est positif.

En s'intéressant aux services intensifs en connaissances (SIC), NÄHLINDER et HOMmEN (2002) confirment cette tendance, pour la Suède, sur la base de 1'ECI 2. Les innovations dans les SIC seraient ainsi positivement corrélées à une croissance de l'emploi comme des qualifications. Ces résultats ne suffisent pas à remettre en cause l'hypothèse récurrente selon laquelle les services seraient essentiellement créateurs d'emplois de faible qualification. Les SIC ne sont pas représentatifs de l'ensemble des services. Compte tenu de leur objet particulier, ils ont tendance (indépendamment même de la relation à l'innovation) à recruter des personnels fortement qualifiés.

Pour l'Allemagne, Peters (2004) s'appuie sur l'ECI 3 pour tenter d'évaluer l'impact sur l'emploi des innovations de produit et de process. La démarche se distingue des précédentes dans la mesure où ces deux types d'innovation ne sont pas envisagés comme des catégories homogènes. L'analyse distingue en effet deux types d'innovation de produit différents selon leur degré de nouveauté (nouveauté pour le marché, nouveauté pour l'entreprise) et deux types d'innovations de processus (d'une part, les technologies de rationalisation, et d'autre part, les innovations de processus qui visent d'autres objets que la rationalisation: par exemple, l'amélioration de la qualité, les exigences réglementaires...). L'analyse économétrique indique que les innovations de produit (qu'il s'agisse de nouveauté pour le marché ou d'imitations, sans différence significative entre les deux) ont un impact net positif sur l'emploi au niveau de la firme. Ce résultat, établi à la fois pour l'industrie et les services, contredit les hypothèses traditionnelles selon lesquelles l'innovation de marché génère davantage d'emplois que l'innovation d'imitation. L'innovation de processus (en particulier sous sa forme d'innovation de rationalisation) exerce des effets négatifs sur l'emploi dans l'industrie. En revanche, durant la période considérée, cette innovation ne semble pas être à l'origine d'une réduction significative de la maind'œuvre, dans les services.

Ces exercices statistiques ne doivent pas nous faire oublier les nombreux problèmes méthodologiques qui en atténuent la portée. En effet, les données des ECI sont obtenues sur la base des indicateurs du Manuel d'Oslo (OCDE, 1997). Or, ces indicateurs sont restrictifs (DJellal, Gallouj, 2000). Ils ne s'intéressent qu'à l'innovation technologique. Un autre problème est celui de la définition de la compétence (et donc de l'appréciation de son évolution positive ou négative au sein d'une entreprise). Les enquêtes ont probablement tendance à sous-évaluer les conséquences positives de l'innovation sur la qualification. Les directives du Manuel de Canberra (OCDE, 1995) qui ne considèrent comme fortement qualifiés que les employés qui ont une formation post secondaire ne paraissent pas satisfaisantes à cet égard.

La question de la distinction entre innovation de produit et innovation de process est ici importante, 
dans la mesure où ces deux formes d'innovation ont, en théorie, des impacts différents sur l'emploi. En effet, l'innovation de processus, dans son acception traditionnelle, consiste à substituer du capital au travail. Elle a donc un effet destructeur d'emplois. L'innovation de produit consiste à introduire des qualités nouvelles sur le marché et donc à susciter une demande nouvelle. Elle contribue à la conquête de nouveaux marchés, autrement dit, à l'accroissement de la production et de l'embauche. Elle est par conséquent créatrice d'emplois. C'est, selon Katsoulacos (1984), cette succession de périodes dominées par l'innovation de produit ou l'innovation de process qui explique les fluctuations du chômage. Même dans l'industrie, cette distinction doit néanmoins être utilisée avec prudence. Ainsi, la nature «produit» ou «process » d'une innovation n'est pas une caractéristique technique intrinsèque. Elle peut dépendre des usages. Dans le domaine des biens d'équipement (celui des machines-outils par exemple), une innovation de produit pour un secteur (le secteur producteur) devient généralement une innovation de process pour un autre secteur (celui qui l'adopte ou l'utilise), avec des effets différents sur l'emploi pour un même système.

Dans les services, cette distinction est difficile à opérer. En excluant les formes non technologiques de l'innovation (dont une bonne partie sont vraisemblablement des innovations non technologiques de produit, par exemple, les produits financiers, les produits d'assurance, il est probable que les régressions économétriques effectuées mettent davantage l'accent sur les innovations de process (des systèmes techniques introduits dans la prestation de service). Par ailleurs, une conception moins matérielle et technologique de l'innovation de process (par exemple, l'innovation méthodologique dans le conseil) peut, dans certains cas, obscurcir la relation innovation-emploi, dans la mesure où l'innovation méthodologique peut avoir des objectifs différents : celui d'économiser du temps en rationalisant et coordonnant les actions et en réduisant les aléas («effet process»), mais aussi celui de conférer un squelette au «produit-service», voire d'ajouter des fonctionnalités, des qualités à un produit (par exemple, un processus de livraison à domicile). C'est, dans ce cas, plutôt l'«effet produit» qui intervient.

\section{Le modèle du cycle inversé et la question de l'emploi}

BARRAS (1986) est à l'origine d'un modèle simple et suggestif qui constitue une théorie de la diffusion de l'innovation technologique d'origine industrielle dans les services. Il décrit le cycle de l'innovation dans les services comme l'inverse du cycle industriel traditionnel formalisé par ABERNATHY et UTTERBACK (1978). Ce cycle inversé, induit par les différentes vagues d'informatisation, articule successivement une phase d'innovation de process incrémentale, une phase d'innovation de process radicale et une phase d'innovation de produit ( $c f$. tableau 2).

$$
\text { Tableau } 2
$$

\section{Le cycle du produit inversé, un cycle de l'emploi}

\begin{tabular}{|l|l|l|}
\hline $\begin{array}{l}\text { Étapes } \\
\text { du cycle }\end{array}$ & $\begin{array}{l}\text { Forme d'inno- } \\
\text { vation domi- } \\
\text { nante }\end{array}$ & $\begin{array}{l}\text { Impacts du progrès technique } \\
\text { sur les facteurs de production }\end{array}$ \\
\hline $\begin{array}{l}\text { Première } \\
\text { étape }\end{array}$ & $\begin{array}{l}\text { Innovation } \\
\text { de process } \\
\text { incrémentale }\end{array}$ & $\begin{array}{l}\text { Progrès technique qui épargne } \\
\text { du travail et augmente la } \\
\text { quantité de capital utilisée }\end{array}$ \\
\hline $\begin{array}{l}\text { Deuxième } \\
\text { étape }\end{array}$ & $\begin{array}{l}\text { Innovation } \\
\text { de process } \\
\text { radicale }\end{array}$ & $\begin{array}{l}\text { Progrès technique neutre vis- } \\
\text { à-vis du travail et favorisant } \\
\text { l'augmentation de la quantité } \\
\text { et de la qualité et du capital }\end{array}$ \\
\hline $\begin{array}{l}\text { Troisième } \\
\text { étape }\end{array}$ & $\begin{array}{l}\text { Innovation } \\
\text { de produit }\end{array}$ & $\begin{array}{l}\text { Progrès technique augmentant } \\
\text { la qualité du capital et épar- } \\
\text { gnant celui-ci (utilisant du } \\
\text { travail) }\end{array}$ \\
\hline
\end{tabular}

Les innovations de process incrémentales apparaissent dans le back-office des organisations. Elles obéissent à une logique d'efficience et de réduction des coûts. Il s'agit, par exemple, de l'enregistrement informatisé des polices d'assurance, de l'informatisation des registres du personnel et des salaires. Dans cette première phase, le cycle inversé est caractérisé par un progrès technique qui épargne le travail et augmente la quantité de capital. Dans les secteurs de services caractérisés par une forte croissance de la demande, le biais «destructeur d'emplois » du progrès technique peut être masqué. Cependant, comme le note BARRAS (1986), c'est dans les secteurs affectés par une saturation, voire un rétrécissement des marchés, que la tendance à l'introduction de technologies destructrices d'emplois est la plus forte.

Les innovations de process radicales concernent essentiellement le front office et elles visent l'amélioration de la qualité. Il s'agit, par exemple, des enregistrements «en direct» des polices dans les bureaux des compagnies d'assurance, de l'installation de guichets automatiques de banque. L'amélioration de la qualité induit une certaine extension des marchés. Dans cette seconde phase du cycle, le progrès technique (en valeur nette) est plus neutre quant au facteur travail et il a un effet équivalent d'augmentation de la qualité et de la quantité du capital.

Les innovations de «produit» quant à elles sont encore relativement rares. La banque à domicile en est la meilleure illustration. Ces innovations visent moins l'efficacité ou la différenciation des produits que la conquête de nouveaux marchés. Elles ont donc un effet positif sur l'emploi. Elles sont associées à un progrès technique qui augmente la qualité du capital tout en épargnant celui-ci, c'est-à-dire en utilisant du travail. 
On constate ainsi que le cycle inversé du produit est aussi un cycle de l'emploi : dans les services, l'innovation commence par être destructrice d'emplois avant d'en être créatrice mais, le modèle du «cycle inversé» et ses implications sur l'emploi achoppent sur sa conception particulière du produit et de la distinction entre produit et process. Par ailleurs, qu'elle soit de produit ou de process, l'innovation envisagée par Barras est une innovation technologique. Ainsi, certaines formes de l'innovation sont exclues du modèle, alors qu'on peut faire l'hypothèse qu'elles exercent un important effet «créateur d'emplois». C'est le cas de l'innovation de produit envisagée, par exemple, dans la banque et l'assurance, dans le sens d'un nouveau produit financier ou d'un nouveau contrat. Le biais technologique du cycle inversé de Barras introduit donc un biais dans l'analyse des impacts de l'innovation sur l'emploi.

\section{Les approches taxonomiques}

Les approches en termes de taxonomies sectorielles ont, a priori, l'intérêt de pouvoir permettre de déplacer l'analyse au niveau sectoriel, et par conséquent de mieux appréhender certains phénomènes de «compensation». Mais, ces taxonomies ne s'intéressent pas explicitement aux impacts des différentes trajectoires d'innovation sur l'emploi. Ainsi, par exemple, dans la taxonomie de SoEte et Miozzo (1990), comme dans celle de PavitT (1984), la taille de la firme (évaluée par ses effectifs) est un des déterminants structurels et non une variable dépendante de l'évolution le long de la trajectoire.

Cependant, dans la mesure où ces taxonomies s'appuient sur la distinction fondamentale entre innovation de produit et innovation de process, et sur la part relative de chacun de ces types dans un secteur donné, on peut faire l'hypothèse que, toutes choses égales par ailleurs, les trajectoires sectorielles correspondantes où domine l'innovation de produit sont créatrices d'emplois, tandis que les trajectoires sectorielles dominées par l'innovation de process sont destructrices d'emplois. On constate ainsi que, dans la taxonomie de Pavitt, tous les services appartiennent à la catégorie "dominée par les fournisseurs ", ce qui signifie qu'ils adoptent des innovations de process élaborées dans d'autres secteurs. Toutes choses égales par ailleurs, l'innovation dans les services est ainsi envisagée comme ayant un effet «destructeurs d'emplois», ce qui est problématique (même si des effets de compensations peuvent être en jeu et que d'autres facteurs que l'innovation interviennent) au regard des statistiques de l'emploi. La taxonomie de Soete et Miozzo (qui distingue, rappelons-le, dans le domaine des services: 1) les firmes dominées par les fournisseurs; 2) les réseaux physiques; 3) les réseaux informationnels; 4) les fournisseurs spécialisés et fondés sur la science) est, de ce point de vue, plus satisfaisante dans la mesure où elle introduit aussi des trajectoires où l'innovation de produit est domi- nante. C'est le cas, par exemple, des fournisseurs spécialisés et fondés sur la science.

Pour intéressantes qu'elles soient ces taxonomies sectorielles sont abstraites. Evangelista (2000b) ainsi que Evangelista et Savona (2003), en s'appuyant sur un certain nombre d'indicateurs issus de l'ECI 2 (pour l'Italie voir tableau 1), proposent une taxonomie sectorielle plus concrète et opérationnelle, qui est en mesure de permettre d'envisager une analyse statistique de la relation innovationemploi au niveau sectoriel.

Cette taxonomie distingue trois modèles sectoriels d'innovation dans les services:

1) les «utilisateurs de technologies». Cette catégorie regroupe les branches les plus traditionnelles des services à savoir le commerce de détail, l'hôtellerie, la restauration, le transport, le nettoyage, etc. Il s'agit de secteurs faiblement innovateurs et dépendants des fournisseurs industriels de technologies;

2) les « utilisateurs de TIC». Ces secteurs sont caractérisés par leur usage intensif des TIC. Il s'agit, en particulier, des banques, des compagnies d'assurance et autres services financiers, du commerce de gros, de la publicité, etc.;

$3)$ les «secteurs fondés sur les sciences et techniques ». On compte dans ce secteur la R-D, l'ingénierie, les conseils techniques, les services informatiques. Ce sont des secteurs caractérisés par leur forte activité d'innovation.

Le travail statistique concernant l'impact de l'innovation sur l'emploi effectué sur la base de cette taxonomie aboutit aux résultats suivants:

1) dans les firmes fondées sur les S-T, l'innovation a un effet globalement positif sur l'emploi. On constate par ailleurs une importante tendance à la substitution de la main-d'œuvre très qualifiée à la main-d'œuvre peu qualifiée. Le changement technique a donc un biais de compétence;

2) dans le secteur des utilisateurs de TIC, on constate un effet négatif de l'innovation sur l'emploi ainsi qu'un biais de compétence important. Autrement dit, l'introduction de TIC dans les banques, l'assurance, les services postaux, etc. exerce un effet destructeur d'emplois. Le recrutement d'une main-d'œuvre qualifiée (consécutive à l'introduction de nouveaux services) n'a pas été en mesure de compenser la destruction d'emplois de faible qualification;

3) dans le secteur des utilisateurs de technologies, l'innovation (c'est-à-dire l'adoption de nouveaux systèmes de transports, par exemple, mais aussi de nouveaux systèmes informatiques) a des conséquences négatives (modérées) sur le volume d'emplois ainsi qu'un biais de compétence (modéré). On constate ainsi que la tendance au remplacement d'une main-d'œuvre moins qualifiée par une main-d'œuvre plus qualifiée est une caractéristique générale pour l'ensemble du secteur des services. 
Les questions des modèles nationaux d'économies et de sociétés de services (GADREY, 2005) et de systèmes nationaux de production et d'innovation (AMABLE et alii, 1997) entretiennent d'évidentes relations avec ces typologies des trajectoires technologiques sectorielles. En effet, la nature des trajectoires technologiques et leurs conséquences sur la quantité et la qualité de l'emploi dépendent fondamentalement de la configuration du modèle ou du système, c'est-à-dire en particulier des secteurs qu'il privilégie. Ainsi, en mettant l'accent sur les structures quantitatives et qualitatives de l'emploi dans les services, GADREY (2005) met en évidence quatre «mondes»d'économies et de sociétés de services: libéral, nordique, continental et familialiste. Les critères à l'origine de cette typologie sont les suivants : 1) le poids relatif des services personnels et commerciaux, d'une part, et des services sociaux, au sens large, d'autre part;2) la qualité de ces emplois; 3) le poids relatif du marchand et du non-marchand.

Plus généralement, il semble que la relation entre l'innovation et l'emploi (en particulier dans les services) peut difficilement être envisagée indépendamment d'une réflexion sur les régimes de croissance (BOYER, 2002). Dans une réflexion consacrée au potentiel de croissance et de productivité des économies développées contemporaines (économies caractérisées à la fois par un niveau élevé de tertiarisation et des régimes de croissance appuyés sur les vagues des NTIC), Pетіт (2002) considère que deux facteurs importants relatifs au tertiaire jouent un rôle dans l'exploitation de cette nouvelle croissance: la capacité des pays à transformer les services sociaux et les services aux ménages (en particulier par l'innovation, la qualification et la professionnalisation), leur capacité à faire du secteur tertiaire complexe un pôle d'innovation et de support du changement et de l'innovation.

\section{La question de l'emploi absente des approches servicielles}

On distingue en général deux groupes d'approches servicielles : 1) les travaux typologiques consacrés à différents types de services; 2) les théories locales de l'innovation adaptées à des secteurs de service particuliers. La question de l'emploi est rarement abordée par ces travaux, dont les efforts portent essentiellement sur la recherche de spécificités éventuelles de l'innovation dans les services, tant dans sa forme que dans ses modes d'organisation ou sa dynamique historique (GAllou, 2002). Il s'agit ici moins de rendre compte de résultats établis par la littérature, que d'ouvrir un certain nombre de pistes de réflexion méritant d'être explorées.

\section{Roue de la distribution et roue de l'emploi, théorie de l'accordéon et emplois en accordéon?}

Les spécialistes du commerce ont tenté d'élaborer des modèles théoriques permettant de comprendre la dynamique de l'innovation dans ce secteur. Deux modèles ont connu un succès important. Il s'agit du modèle de la « roue de la distribution» (MCNAIR, 1958) et de la «théorie de l'accordéon » (Hollander, 1966).

Selon McNair, l'innovation dans les formats de magasins suit une trajectoire circulaire dans laquelle les «formats en place» sont concurrencés par des «formats nouveaux », moins coûteux car gérés selon une logique de rationalisation des installations, de restriction forte des assortiments et de réduction des services.

Les ressorts de la roue de la distribution peuvent ainsi être résumés de la manière suivante:

1) tous les formats de distribution nouveaux apparaissent sous une forme «bon marché». Leur succès tient essentiellement aux bas prix qu'ils pratiquent, ces bas prix étant le résultat d'une politique d'assortiments et de services réduits, visant des volumes de vente élevés ;

2) le succès de ce format initial attire des concurrents. Pour leur faire face, ce format aura tendance à mettre en œuvre des stratégies de différenciation, qui conduiront inévitablement à l'alourdissement des charges d'exploitation, et se répercuteront sur les prix;

3) cet «embourgeoisement» de la formule amorce un mouvement de rotation de la roue. La formule quitte ainsi la position «discounter» et se trouve à la merci d'un nouvel entrant proposant une formule plus «sommaire» et moins coûteuse.

Selon une perspective voisine, la «théorie de l'accordéon» (Hollander, 1966) se propose d'expliquer la succession des formats de magasin par la structure et la dynamique des assortiments. Elle met ainsi en évidence une alternance historique (d'où l'image de l'accordéon) de formats caractérisés par un assortiment large et non spécialisé, puis de formats organisés autour d'un assortiment étroit et spécialisé.

Ces théories locales de l'innovation commerciale ne s'intéressent pas directement à la question de l'emploi. Mais, tout au moins au niveau microéconomique, si l'on ne tient pas compte des phénomènes de compensation éventuels (en particulier des conséquences en termes de parts de marché des formules commerciales de type discount), on peut probablement associer des niveaux d'emplois à chaque position de la roue ou de l'accordéon. En effet, les versions discount sont associées à des niveaux d'emploi faibles, tandis que les versions embourgeoisées nécessitent un accroissement de l'emploi. De même, dans sa position «assortiment étroit et spécialisé», l'accordéon traduit des formats «destructeurs 
d'emplois », tandis que dans sa position «assortiment large non spécialisé», l'accordéon correspond à des formats «créateurs d'emplois».

\section{Les typologies de l'innovation dans les services issues d'investigations empiriques}

Les approches dites servicielles ont multiplié les investigations empiriques afin de mettre en évidence l'existence de formes particulières d'innovation dans les services. Ces investigations dénoncent unanimement une certaine myopie des conceptions technologistes, qui privilégient l'innovation technologique au détriment d'autres formes d'innovation.

Des travaux de ce type ont été réalisés pour différentes catégories de service: services de conseil, services financiers et d'assurance, services de distribution, hôtellerie-restauration, services de proximité, etc. La question de l'emploi dans sa relation à l'innovation y est rarement envisagée. Ce n'est pas surprenant, dans la mesure où les efforts de recherche sont concentrés sur la question difficile de la nature hétérogène de l'innovation, associée à la nature hétérogène du produit. En effet, les types de «produits» sont extrêmement variables d'une activité de service à l'autre. Ainsi, l'innovation de produit n'a pas le même contenu pour un service hôtelier, un service de conseil ou un service financier. La difficulté d'application des définitions traditionnelles semble augmenter avec les services dont le support est l'information, la connaissance ou l'individu.

Cependant, il est possible de faire des hypothèses sur la capacité à créer ou à détruire des emplois des différentes formes d'innovation mises en évidence dans la littérature servicielle, en prenant comme point de référence la distinction traditionnelle entre l'innovation de produit et l'innovation de process. En effet, rappelons-le, toutes choses égales par ailleurs, c'est-à-dire sans tenir compte des phénomènes de compensation positifs ou négatifs (cannibalisation des produits anciens par les innovations de produit, effet prix pour les innovations de process, etc.), on admet généralement que les innovations de produit sont favorables à l'emploi dans la mesure où elles augmentent la variété des biens et des services et où elles ouvrent de nouveaux marchés. Les innovations de process quant à elles sont destructrices d'emplois (au niveau de la firme tout au moins et à un moment donné) dans la mesure où elles visent à améliorer l'efficacité de la production de biens ou de services, donc à substituer du capital au travail. On peut tenter d'évaluer les potentiels «créateurs d'emplois» ou «destructeurs d'emplois» d'un certain nombre de formes particulières d'innovation proposées dans la littérature servicielle. Dans les analyses qui suivent, l'enjeu théorique principal n'est pas, à ce stade, il faut le noter, la question de l'impact sur l'emploi (puisque nous partons de l'hypothèse que, toutes choses égales par ailleurs, les innovations de produit et les innovations de process engendrent respectivement des effets créateurs et des effets destructeurs d'emplois), mais celle de l'identification et de la désignation du produit et du process. Les formes d'innovation proposées par la littérature servicielle contournent le plus souvent la distinction innovation de produit-innovation de process, mais on peut tenter d'apprécier lequel des effets «produit» ou «process» semble dominer dans chaque type d'innovation. Nous allons tenter de réaliser cet exercice pour trois typologies de l'innovation associées à trois secteurs de services différents.

\section{Les services intensifs en connaissances (SIC)}

Sur la base d'une investigation empirique approfondie dans de nombreuses firmes de conseil appartenant à différents secteurs, Gallous $(1994,2002)$ propose de distinguer trois types d'innovation: l'innovation ad hoc, l'innovation de «nouveau champ d'expertise », l'innovation de formalisation.

- L'innovation ad hoc est définie comme une solution permettant d'éclairer, avec un certain degré de nouveauté, le problème (juridique, organisationnel, stratégique, technique...) d'une firme sans qu'il soit nécessairement possible de la transférer (totalement) à d'autres (GALlouj, 2002). Il s'agit en quelque sorte d'une innovation incrémentale de produit dans laquelle le produit n'est pas une entité tangible, mais une solution intellectuelle à un problème d'entreprise. On peut donc faire l'hypothèse que, toutes choses égales par ailleurs, c'est ici l'effet «produit» («créateur d'emplois ») qui intervient, dans la mesure où l'innovation ad hoc (et cette aptitude à produire des solutions innovantes) est à l'origine de la conquête de nouveaux clients et du renouvellement des demandes des clients actuels. On fait ainsi l'hypothèse que le recours au conseil s'explique davantage par les niveaux d'expertise des consultants que par le coût du conseil.

- L'innovation de «nouveau champ d'expertise» décrit l'accumulation de connaissances-input relatives à des champs de connaissances émergents (Internet en est aujourd'hui un bel exemple), avec l'idée de fournir des prestations (connaissances-output) relatives à ces champs nouveaux (Gallouj, 2002). Par analogie avec les catégories de SCHUMPETER (1961), et dans la mesure où, pour les SIC, la connaissance est à la fois l'input et l'output, on peut dire que l'innovation de nouveau champ d'expertise recouvre à la fois 1) de nouveaux produits;2) de nouveaux marchés; 3) de nouvelles sources de matières premières. Ce type d'innovation, contrairement au précédent, est radical. C'est de nouveau ici l'effet «produit» («créateur d'emplois ») qui est clairement dominant. L'innovation de «nouveau champ d'expertise» peut se traduire par la création de départements nouveaux dans les firmes existantes, voire par la création d'entreprises nouvelles. 
- L'innovation de formalisation désigne un ensemble hétérogène de mécanismes qui visent à rendre le service moins flou, moins «immatériel». Ce objectif peut être atteint par deux types de mécanismes différents qui peuvent se conjuguer: 1) des mécanismes tangibles: par exemple, l'introduction de systèmes techniques dans la formule de service; 2) des mécanismes intangibles: l'introduction de méthodes, c'est-à-dire de «scripts» décrivant la distribution des rôles dans cette véritable « représentation » (théâtrale) qu'est la prestation de service; la conception et l'usage de boîtes à outils comportant des instruments analytiques structurant les raisonnements et les comportements (par exemple, les matrices du BCG); la mise en place d'une organisation qui incorpore le service immatériel.

Il serait erroné d'envisager cette forme d'innovation, par opposition aux deux formes d'innovation (de produit) précédentes, comme une innovation de processus, autrement dit, de réduire la formalisation à une activité de rationalisation. L'innovation de «formalisation» a un impact probablement indéterminé sur l'emploi ou plus exactement un impact variable, dépendant du type de formalisation réalisé. On pourrait considérer grossièrement que les mécanismes tangibles exercent plutôt un effet «process», tandis que les mécanismes intangibles exercent un effet «produit». Autrement dit, si elle consiste en l'introduction de systèmes techniques, alors la formalisation génère des économies de main-d'œuvre (effet process), mais si elle revêt des formes immatérielles (visant à rendre le produit plus visible sans effet significatif sur l'efficacité de sa réalisation), alors c'est l'effet produit («créateur d'emplois ») qui est dominant. Mais en réalité, certains mécanismes tangibles génèrent également des effets «produit», alors que l'effet «process» est également présent dans les mécanismes intangibles.

\section{Les services d'assurance et les services financiers}

Dans l'assurance et les services financiers, la typologie proposée (GADREY, GALlouJ, 1994) peut paraître a priori simple et facile à interpréter, dans la mesure où elle dissocie pour l'essentiel des innovations de produit et des innovations de process, qui sont ainsi désignées ( $c f$. tableau 3 ). Cependant, l'exercice d'interprétation est plus difficile qu'il n'y paraît. En effet, pour faciliter l'analyse, les différents types d'innovations sont présentés ici de manière séparée. En réalité, ils sont bien souvent indissociables dans leur production comme dans leurs effets. Ainsi, de nombreuses innovations de process et de produit ne sont que les deux facettes d'un même phénomène et les innovations de process et d'organisation sont souvent indissociables. Autrement dit, une même innovation (ou convention d'innovation) est le terrain d'influences contradictoires en termes de création d'emplois, dont il n'est pas aisé d'effectuer le bilan.
Les catégories A, B, C (innovations de produitsservices, innovations architecturales, innovations de modification du produit-service) sont néanmoins bien des innovations de produits d'un type certes particulier, puisqu'elles sont immatérielles (nouveaux contrats, nouveaux services). Elles constituent le cœur de l'activité d'assurance, mais échappent pour l'essentiel, par exemple, au modèle de BARrAs (1986), qui retient, comme nous l'avons déjà souligné, une définition très restrictive des «nouveaux produits». Leur effet sur l'emploi est ainsi a priori (toutes choses égales par ailleurs) de type «créateur d'emplois». En revanche, dans les catégories D1, D2, D3, D4 (innovations de process, d'organisation, de méthodes et de gestion), c'est l'effet de proccess («destructeur d'emplois») qui est mis en valeur. Néanmoins, il est clair que les effets des différentes innovations sur l'entreprise, ses métiers, ses qualifications, ses emplois, etc. ne peuvent être isolés, mais doivent être appréhendés de manière systémique.

\section{Les services de distribution}

Dans les services de distribution, DupuIs (1998) propose de distinguer les quatre formes suivantes d'innovation: 1) l'innovation de concept; 2) l'innovation de flux; 3) l'innovation organisationnelle; 4) l'innovation architecturale.

L'innovation de concept peut être définie comme la combinaison d'un format de distribution et d'un positionnement différenciateur. C'est ainsi l'effet «produit» qui y domine, et donc, au niveau microéconomique, l'effet «créateur d'emplois».

Les innovations de flux visent à réduire les coûts en optimisant différents types de flux (physiques, financiers ou d'informations). De même, les innovations organisationnelles (internes ou externes) ont pour objectifl'amélioration du fonctionnement et de la rentabilité des réseaux. Si l'on neutralise les effets de compensations éventuels, les innovations de flux et les innovations organisationnelles exercent donc essentiellement un effet «destructeur d'emplois ».

Les innovations architecturales, enfin, sont définies comme la combinaison cohérente des différents types précédents d'innovation (de concept, de flux et organisationnelle). Leur effet sur l'emploi est ainsi difficile à déterminer a priori dans la mesure où il combine et est la résultante des effets destructeurs et créateurs d'emplois.

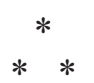

La question de l'innovation dans les services dans ses relations à l'emploi est une question fondamentale, qui paradoxalement n'occupe pas la place qui lui revient dans la littérature économique. Dans cet article, nous avons, à titre essentiellement programmatique, envisagé cette question, en tentant de l'ar- 
Tableau 3

Les principales formes d'innovation dans l'assurance

\begin{tabular}{|c|c|c|c|}
\hline $\begin{array}{l}\text { Types } \\
\text { d'innovation }\end{array}$ & \multicolumn{2}{|c|}{ Sous catégories } & Définition \\
\hline \multirow{4}{*}{$\begin{array}{l}\text { A : } \\
\text { Innovations } \\
\text { de « produits- } \\
\text { services » }\end{array}$} & \multicolumn{2}{|c|}{ A1 : Innovations de produits-services } & Service, concept, contrat, nouveau \\
\hline & \multirow{3}{*}{$\begin{array}{l}\text { A2 : Innovations } \\
\text { de produits- } \\
\text { services sur } \\
\text { mesure }\end{array}$} & $\begin{array}{l}\text { 1) Innovations sur mesure } \\
\text { d'adaptation }\end{array}$ & Adaptation à un client d'un contrat standard \\
\hline & & $\begin{array}{l}\text { 2) Innovations «sur mesure } \\
\text { total» }\end{array}$ & $\begin{array}{l}\text { Conception d'un contrat réellement spécifique pour un client } \\
\text { donné }\end{array}$ \\
\hline & & $\begin{array}{l}\text { 3) Couverture de risques } \\
\text { spéciaux }\end{array}$ & $\begin{array}{l}\text { Couverture d'un risque nouveau concernant des populations } \\
\text { statistiques réduites }\end{array}$ \\
\hline \multirow{2}{*}{$\begin{array}{l}B \text { : Innovations } \\
\text { architecturales }\end{array}$} & \multicolumn{2}{|c|}{$\begin{array}{l}\text { B1 : Innovation d'association de « produit- } \\
\text { services » }\end{array}$} & Combinaison de produits-services existants \\
\hline & \multicolumn{2}{|c|}{$\begin{array}{l}\text { B2 : Innovation de dissociation de « produit- } \\
\text { services » }\end{array}$} & $\begin{array}{l}\text { Isolation d'un fragment de produit-service pour la vente } \\
\text { séparée }\end{array}$ \\
\hline \multicolumn{3}{|c|}{$\mathrm{C}$ : Innovations de modification du produit-service } & $\begin{array}{l}\text { La formule de base est inchangée, mais certaines spécifica- } \\
\text { tions ou options changent }\end{array}$ \\
\hline \multirow{4}{*}{$\begin{array}{l}\text { D: } \\
\text { Innovations } \\
\text { de process, } \\
\text { d'organisation, } \\
\text { de méthodes et } \\
\text { de gestion }\end{array}$} & \multicolumn{2}{|c|}{$\begin{array}{l}\text { D1 : Innovations accompagnant les innovations } \\
\text { de produits-services }\end{array}$} & $\begin{array}{l}\text { Innovation de process et d'organisation consécutive à une } \\
\text { innovation de produits-services des types A, B ou C et indis- } \\
\text { sociable d'elles }\end{array}$ \\
\hline & \multicolumn{2}{|c|}{$\begin{array}{l}\text { D2 : Innovation pour un produit-service identique } \\
\text { dans ses spécifications formelles et dans son } \\
\text { mode de prestation }\end{array}$} & $\begin{array}{l}\text { Modification importante du processus (technologie, organi- } \\
\text { sation du travail) pour un service final inchangé }\end{array}$ \\
\hline & \multicolumn{2}{|c|}{$\begin{array}{l}\text { D3 : Innovations pour un produit-service iden- } \\
\text { tique dans les spécifications formelles mais } \\
\text { amélioré dans la réalisation concrète, la qualité } \\
\text { perçue, le mode de commercialisation }\end{array}$} & $\begin{array}{l}\text { Modification importante du processus (technologie, organi- } \\
\text { sation du travail) pour un produit « formellement » identique } \\
\text { mais de meilleure qualité }\end{array}$ \\
\hline & \multicolumn{2}{|c|}{ D4 : Innovations de gestion } & $\begin{array}{l}\text { Innovations portant sur la gestion financière, actuarielle, } \\
\text { juridique, des ressources humaines, etc. }\end{array}$ \\
\hline
\end{tabular}

ticuler aux principaux travaux empiriques ou théoriques consacrés à l'innovation dans les services. $\mathrm{Si}$, bien qu'elle soit unanimement considérée comme fondamentale, cette question est relativement peu exploitée, c'est que l'une des variables de la relation (à savoir la question de l'innovation dans les services) n'est pas encore clairement élucidée sur le plan théorique. En effet, le caractère encore flou de la nature et du statut de l'innovation dans les services conduit à considérer comme hasardeuse toute tentative d'analyse des conséquences de cette innovation sur l'emploi.

Il s'agit-là pourtant d'un champ de recherche prometteur, important sur le plan théorique et des politiques publiques qu'il faudrait explorer davantage, à la fois sur le plan micro, méso et macroéconomique, que ce soit par le biais de la construction théorique déductive ou de l'analyse inductive qualitative ou statistique. Les deux axes généraux de recherche que nous avons explorés dans cet article peuvent fournir un premier cadrage pour une telle réflexion.

Plusieurs autres champs intéressants, que nous n'avons pas évoqués ici, mériteraient une analyse spécifique. Il s'agit, tout d'abord, de la question de l'innovation non plus dans les services, mais par les services, c'est-à-dire du rôle que peuvent jouer certaines activités de services (en particulier les
SIC) dans l'innovation et l'emploi de leurs clients. Il n'existe pas, à notre connaissance, de travaux statistiques consacrés au lien entre l'innovation induite par les SIC et l'emploi chez leurs clients. C'est une piste de recherche intéressante à explorer, qu'il faudrait envisager en construisant des questionnaires spécifiques.

Il s'agit ensuite de la question de l'entrepreneuriat dans les services dans ses relations à l'emploi. En effet, si la question de l'entrepreneuriat dans ses relations aux services n'est pas nouvelle, dans la mesure où l'essentiel des créations d'entreprises s'opère dans le secteur tertiaire, en revanche, celle de l'entrepreneuriat dans sa relation à l'innovation dans les services est rarement envisagée.

On peut également aborder la question de la relation innovation emploi en inversant les termes de la causalité, c'est-à-dire en se consacrant à l'analyse des conséquences de la nature du marché du travail sur l'innovation et la productivité. Ainsi, sans s'intéresser, il est vrai, aux services exclusivement, KLEINKNECHT et alii (2006) montrent que la flexibilisation (externe) des marchés du travail a des effets négatifs importants sur les activités d'innovation au sein des firmes, dans la mesure où elle dégrade la confiance et la loyauté nécessaires au développement de l'innovation et à l'amélioration de la productivité. 


\section{Bibliographie}

Abernathy W., Utterback J. (1978), "Patterns of industrial innovation", Technology Review, 80, p. 41-47.

Amable B., Barré R., Boyer R. (1997), Les systèmes d'innovation à l'ère de la globalisation, Economica.

Appelbaum E. (1987), “Technology and the redesign of work in the insurance industry", in Drygulski White B. (ed) Women, work and technology. Transformations, University of Michigan Press.

BARRAS R. (1986), "Towards a theory of innovation in services", Research Policy, 15, p. 161-173.

BAumOL W. (1967), "Macroeconomics of unbalanced growth", Amercian economic review, 2, 415-426.

Bell D. (1976), Vers la société post-industrielle, Laffont.

Bertrand O. (1988), «Qualité et hétérogénéité des emplois de services », Formation et Emploi, n 23, p. 1929.

Bertrand O. Noyelle T. (1987), «L'emploi dans les banques et assurances: comparaisons internationales et perspectives d'évolution», Économie et Humanisme, $\mathrm{n}^{\circ} 295$.

Bluestone B., Harrison B. (1986), The Great American Job Machine, Report for the Joint Economic Committee, December.

Boyer R. (2002), La croissance, début de siècle: de l'octet au gène, Albin Michel.

Cossalter C., Hezard L. (1983), Nouvelles perspectives de l'informatisation dans les banques et les assurances, CEREQ.

Devetter F.-X. (2001), L'économie de la disponibilité temporelle au travail, thèse de doctorat, université de Lille 1.

Djellal F. (2002), "Innovation trajectories in the cleaning industry", New Technology and Employment, vol. 17, $\mathrm{n}^{\circ} 2$, p. 119-131.

Duellal F., Francoz D., Gallouj C., Gallouj F., Jacquin Y. (2003), "Revising the definition of research and development in the light of the specificities of services", Science and Public Policy, 30/6, p. 415-430.

Djellal F., Gallouj F. (2000), «Le casse-tête» de la mesure de l'innovation dans les services: enquête sur les enquêtes ", Revue d'économie industrielle, $\mathrm{n}^{\circ} 93,4 \mathrm{e}$ trimestre, p. 7-28.

Dupuis M. (1998), «L'innovation dans la distribution: ses implications dans les rapports industrie-commerce», Décision Marketing, n 15(3), p. 29-41.

Evangelista R. (2000a), Innovation and Employment in Services: Results from the Italian Innovation Survey, in
Vivarelli M., Pianta M. (eds) The Employment Impact of Innovation: Evidence and Policy, Routledge.

EVANGELISTA R. (2000b), "Sectoral patterns of technological change in services", Economics of innovation and new technology, 9, p. 183-221.

Evangelista R., Savona M. (2003), "Innovation, employment and skills in services: firm and sectoral evidence", Structural Change and Economic Dynamics, 14, p. 449474.

Kleinknecht A., Oostendorp R.M., Pradhan M.P., NAAstepad C.W.M. (2006), "Flexible Labour, Firm Performance and the Dutch Job Creation Miracle", International Review of Applied Economics, vol. 20, $\mathrm{n}^{\circ} 2$, p. 171-187.

Fourastié J. (1949), Le grand espoir du XXe siècle, PUF.

Freeman C., Soete L. (1994), Work for all or mass unemployment? computerised technical change into the twenty-first century, Pinter.

Freeman C., Soete L. (eds) (1987), Technical change and full employment, Basil Blackwell.

Freeman C., Clark J., Soete L. (1982), Unemployment and technical innovation, Pinter.

GADREY J. (2005), Les quatre «mondes» des économies de services développées, Économies et Sociétés, $\mathrm{n}^{\circ} 11-$ 12/2005, p. 1925-1970.

Gadrey J., Gallouj F. (1994), L'innovation dans l'assurance: le cas de l'UAP, UAP et ministère de l'Enseignement supérieur, de la Recherche et de la Technologie.

Gallouj F. (1994), Économie de l'innovation dans les services, L'Harmattan.

Gallouj F. (2002), Innovation in the Service Economy: the New Wealth of Nations, Edward Elgar Publishers.

Hollander S.C. (1966), Notes on the retail accordion, Journal of Retailing, 42(2), p. 24-34.

JANY-CATRICE F. (2004), «Une analyse socio-économique de l'emploi dans l'hôtellerie-restauration en France et aux États-Unis», Économies et sociétés, série Socioéconomie du travail, $\mathrm{n}^{\circ} 23$ (1).

Katsoulacos Y. (1984), "Product innovation and employment”, European Economic Review, 26, p. 83-108.

MAHAR, M. (1992), "Blue collar, white collar: good jobs are vanishing throughout the economy", Barron's, May, 11, 8-24.

McNaIR, M.P. (1958), "Significant trends and developments in the post war period", in A.B. Smith (ed.) Competitive Distribution in a Free High Level Economy 
and its implication for the University, University of Pittsburgh Press, p. 1-25.

NäHlinder J., Hommen L. (2002), Employment and Innovation in Services: Knowledge Intensive Business Services in Sweden, Report for the final meeting and conference of AITEG, Birkbeck College, Londres, 18 au 18 avril.

OCDE (1995) Manuel sur la mesure des ressources humaines consacrées à la science et à la technologie, Manuel de Canberra, Paris.

OCDE (1997), Principes directeurs proposés pour le recueil et l'interprétation des données sur l'innovation technologique, Manuel d'Oslo, Paris.

PavitT K. (1984), Sectoral "Patterns of Technical Change: Towards a Taxonomy and a Theory", Research Policy, $\mathrm{n}^{\circ} 13$, p. 343-373.

Peters B. (2004), Employment effects of different innovation activities : microeconometric evidence, $\mathrm{ZEW}$, dicussion paper $\mathrm{n}^{\circ}$ 04-73.

Petit P. (1995), Employment and Technological Change, in Stoneman P. (ed) Handbook of the economics of innovation and technological change, p. 366-408.
Petit P. (2002), Growth and Productivity in a knowlege based service economy, in Gadrey J., Gallouj F. (eds) Productivity, Innovation and knowledge in services, Edward Elgar.

Petit P., Soete L. (eds) (2001), Technology and the future of european employment, Edward Elgar.

SChumpeter J. (1961), Théorie de l'évolution économique, Librairie Dalloz, Paris.

Soete L., Miozzo M. (1990), Trade and Development in Services: a Technological Perspective, mimeo, MERIT.

Thurow L. (1989), Towards a high-wage, high productivity service sector, Economic Policy Institute, Washington DC.

Vivarelli, Pianta (eds), (2000), The employment impact of innovation: evidence and policy, Routledge.

Vivarelli M. (1995), The economics of technology and employment: Theory and Empirical Evidence, Edward Elgar.

WeBster J. (1996), Shaping women's work. Gender employment and information technology, Longman. 


\title{
Service public de l'emploi et sous-traitance la France au regard des expériences australiennes, britanniques et néerlandaises
}

\begin{abstract}
Céline Gratadour $\left({ }^{*}\right)$ Michèle Mansuy $\left(^{* *}\right)$
L'article analyse les questions principales qui se posent lorsqu'on souhaite recourir à des opérateurs privés pour assurer tout ou partie des missions du service public de l'emploi (SPE). Il synthétise les solutions qui ont pu être retenues dans des pays qui ont poussé le plus loin le recours à des opérateurs externes, l'Australie, les Pays-Bas et à un moindre degré, le Royaume Uni. Voir en annexe page 57 une présentation détaillée des réformes menées dans ces pays. Les auteures tentent de comprendre les principales conditions de la réussite du recours à des opérateurs privés et les difficultés souvent éprouvées par les pouvoirs publics pour réunir ces conditions. Elles s'interrogent sur les mécanismes de gouvernance à mettre en auvre pour garantir la réalisation des missions du SPE, en présentant d'abord à la lumière de la théorie économique les principaux risques associés au recours à des opérateurs privés et en présentant ensuite les mécanismes ex ante et ex post qui leur semblent indispensables pour les éviter. L'article suggère également des réformes structurelles pour arriver à une régulation satisfaisante, en ce qui concerne en particulier la clarification des rôles respectifs des institutions et organismes qui constituent les «trois cercles» du service public de l'emploi.
\end{abstract}

Au cours des années 1990, dans un contexte de chômage élevé et de priorité donnée à la maîtrise des dépenses publiques, les pays membres de l'Union européenne ont développé des politiques visant à rendre les dépenses et les dispositifs de protection sociale plus favorables au retour à l'emploi. De plus, la stratégie d'activation des dépenses a progressivement laissé place à une stratégie d'activation des personnes concernées (JoLY, 2005). Ces politiques de Welfare to Work ont modifié en profondeur la philosophie même de l'intervention publique dans le domaine de l'emploi. La notion de bénéficiaire de politique de l'emploi a progressivement été remplacée par celle d'acteur «coproducteur et coresponsable de son employabilité, et ce faisant, de la réussite des politiques d'emploi et de formation» (BERTHET, 2006). Ainsi, cette activation accrue des politiques de l'emploi a eu des conséquences d'une part sur les attentes du service public de l'emploi (SPE) envers leurs bénéficiaires, dans la mesure où les droits des demandeurs d'emploi ont été renforcés, mais aussi leurs obligations. D'autre part, l'activation a également bouleversé l'organisation du service public de l'emploi.

En effet, l'accent est aujourd'hui mis sur la nécessité pour le SPE d'offrir, en contrepartie des exigences supplémentaires pesant sur les demandeurs d'emploi, des prestations de plus en plus individualisées et adaptées à leurs besoins, grâce notamment au recours à des opérateurs privés spécialisés, à but lucratif ou non lucratif. Cette introduction

(*) Centre d'Économie de la Sorbonne, celine.gratadour@, malix.univ-paris1.fr; celine.gratadour@dares.travail.gouv.fr (**) CERC, michele.mansuy@cerc.gouv.fr d'opérateurs privés dans le champ du service public de l'emploi a été prônée par un grand nombre d'organismes internationaux, en particulier par l'Organisation internationale du travail (OIT), à travers la ratification de la convention 181(1) en 1997, mais aussi par la Commission européenne(2). Ainsi, en Europe, de plus en plus d'opérateurs privés interviennent sur le marché du travail afin d'exercer des missions réservées auparavant aux SPE. La France ne fait pas exception. Le rapport BALMARY (2004) souligne que l'externalisation de prestations d'accompagnement est un processus très développé en France. La mise en œuvre de la loi de cohésion sociale (2005) a eu depuis des conséquences importantes sur l'architecture institutionnelle du SPE, en annulant le monopole formel de l'ANPE en matière de placement et d'accompagnement des demandeurs d'emploi, et de fait en reconnaissant le rôle des opérateurs privés qui contribuent au service public de l'emploi ( $c f$. encadré 1).

(1) Cette ratification constitue une rupture par rapport à la politique traditionnellement prônée par l'OIT. En effet, cette convention reconnaît le rôle productif que peuvent jouer les agences privées d'emploi dans le fait de délivrer des services spécifiques sur le marché du travail tout en s'assurant que les droits des demandeurs d'emploi sont respectés (BARBIER et alii, 2003).

(2) Dans le cadre de la Stratégie européenne pour l'emploi, la Commission européenne a proposé aux États membres un cadre de référence pour améliorer l'efficacité et l'efficience des SPE. Après avoir mis en avant leur rôle central, la Commission a souligné la nécessité pour eux de s'adapter à un environnement en évolution rapide et de définir plus précisément «leur rôle propre et la valeur qu'ils ajoutent au marché, par rapport aux fournisseurs commerciaux et non commerciaux de services $»$. 


\section{Encadré 1}

\section{Les nouveaux contours du SPE français}

La mise en œuvre de la loi de programmation de la cohésion sociale du 18 janvier 2005 a modifié la définition du SPE, qui se compose désormais de plusieurs cercles (Rousseau, 2005). Le premier cercle englobe non seulement les services de l'État chargés de l'emploi, l'ANPE, l'AFPA, mais aussi les organismes paritaires de l'assurance chômage (UNEDIC et ASSEDIC). Un second cercle est constitué par les collectivités territoriales et leurs groupements qui "concourent au SPE » et les missions locales, pour constituer dans chaque bassin d'emploi des maisons de l'emploi. Peuvent en outre «participer au SPE », dans son troisième cercle, tout organisme public ou privé dont «l'objet consiste en la fourniture de services relatifs au placement, à l'insertion, à la formation et à l'accompagnement des demandeurs d'emploi ». II s'agit plus précisément des organismes liés à l'État, des entreprises de travail temporaire ainsi que des agences de placement privées.

Suite à cette reconnaissance explicite du rôle des opérateurs privés dans le domaine de l'emploi, l'ANPE et l'UNEDIC expérimentent conjointement de nouvelles modalités d'accompagnement des demandeurs d'emploi impliquant fortement certains opérateurs privés ( $c f$. encadré 2). Dans le cadre de ces expérimentations, ce ne sont plus seulement des prestations ponctuelles qui sont confiées à des prestataires privés, comme c'était le cas jusque-là, mais des parcours complets d'accompagnement. De ce fait, en France, un pas supplémentaire a été franchi en matière d'implication des opérateurs privés dans l'accompagnement des demandeurs d'emploi.

Alors qu'en France, le recours à des opérateurs privés était jusqu'ici limité, d'autres pays, en particulier l'Australie, les Pays-Bas et le Royaume-Uni leur ont accordé une place plus importante (voir annexe p. 57).

Une telle intervention croissante d'opérateurs privés pour réaliser des missions de service public soulève la question des mécanismes de gouvernance susceptibles de garantir que les missions de SPE sont respectées. Le propos de cet article est de donner à cette question un éclairage externe à la France, en nous appuyant sur les expériences de pays où l'accompagnement des demandeurs d'emploi est confié à des opérateurs externes au SPE depuis plus longtemps (3).

(3) Et, en particulier, sur les enseignements tirés de différentes missions réalisées dans le cadre de la rédaction du sixième rapport du CERC (2005) Aider au retour à l'emploi, missions réalisées au Royaume-Uni, Pays-Bas, Allemagne, Danemark et Suède.
Encadré 2

\section{Les expériences de reclassement de chômeurs de longue durée par des opérateurs privés en France}

Lancées en 2005 et poursuivies en 2006, des expériences pilotes de sous-traitance à des prestataires privés de l'accompagnement de chômeurs indemnisés à fort risque de chômage durable, identifiés par la procédure de profilage (test statistique et entretien professionnel) comme devant recevoir une aide conséquente lors du premier rendez-vous, ont été menées par l'ANPE et I'UNEDIC. Ces expérimentations ont porté sur 9000 allocataires et ont été confiées à cinq opérateurs privés. Comme le souligne le rapport de la Cour des comptes (2006), ces contrats n'ont donné lieu à aucune mesure de publicité ni de mise en concurrence.

Ingeus, une entreprise australienne opérant dans le reclassement de publics jugés difficiles dans plusieurs pays, notamment au RoyaumeUni, a accompagné 6000 chômeurs pour une période maximale de dix mois.

BPI et Altédia, deux entreprises spécialisées dans le reclassement des chômeurs, accompagnent plus de 1000 personnes en reconversion professionnelle ou issues d'entreprises en restructuration.

Adecco, une entreprise de travail temporaire, a suivi 2000 chômeurs à fort risque présumé de chômage de longue durée, dès leur inscription au chômage.

Une plate-forme commune à l'Anpe et l'Association pour l'emploi des cadres (APEC), Étap Carrières, reclasse 600 cadres seniors.

Récemment, I'UNEDIC et l'ANPE ont décidé de prolonger de telles expérimentations en confiant l'accompagnement de 46000 chômeurs à dix-sept opérateurs privés pour un marché de 165 millions d'euros par an.

Dans un premier temps, nous réfléchirons au partage possible des rôles et responsabilités entre opérateurs publics et privés, en analysant plus particulièrement quelles sont les fonctions du SPE qui peuvent être confiées à des opérateurs privés, et de quelle manière. Les mécanismes de gouvernance à mettre en œuvre pour que les missions de service public soient respectées seront présentés dans un second temps. 


\section{Quel partage des rôles et des responsabilités entre opérateurs publics et privés?}

De nombreux pays européens recourent aujourd'hui à des opérateurs privés pour exercer des missions relevant du service public de l'emploi. Un argument justifiant de recourir à de tels opérateurs est d'améliorer l'efficacité et l'efficience du SPE, mais aussi la qualité des services offerts avec notamment une meilleure adaptation aux besoins des demandeurs d'emploi (DE Koning et alii, 1999). Cependant, en fonction des pays, des choix différents ont été effectués concernant l'opérateur impliqué, les missions qui lui sont confiées et les arrangements organisationnels construits entre opérateurs publics et privés (JoLY, 2005).

\section{Quelle fonction de service public d'emploi confier à des opérateurs privés?}

Parmi les trois fonctions généralement associées au service public de l'emploi (l'accompagnement des demandeurs d'emploi, le placement et l'indemnisation), Rousseau (2005) établit une distinction. Selon lui, les missions de placement et d'accompagnement peuvent être confiées à des opérateurs privés ou être soumises à la concurrence. En revanche, l'indemnisation des chômeurs s'apparenterait davantage à une mission d'intérêt général et, à ce titre, ne pourrait pas relever des règles du marché (4).

De plus, il est généralement admis que d'autres fonctions connexes aux missions générales du SPE doivent rester de sa responsabilité:la rédaction des textes et le contrôle de la recherche d'emploi, la conception de l'architecture d'ensemble des systèmes d'information afin d'assurer leur cohérence, etc. (BALMARY et alii, 2004).

En pratique pourtant, la délimitation du champ d'intervention des institutions publiques du SPE et des opérateurs externes se décline différemment d'un pays à l'autre.

À partir des fonctions qui sont confiées à des opérateurs privés et de celles qui restent dans le giron public, Struyven (2004) a ainsi identifié trois configurations principales. La première, illustrée par les Employment Zones au Royaume-Uni, se caractérise par le fait que les fonctions de placement, d'accompagnement et les fonctions d'indemnisation sont mises en œuvre par des opérateurs privés

(4) Ce qui n'implique pas nécessairement qu'elle doit être réalisée par un opérateur à statut public. Cette fonction peut être remplie par les partenaires sociaux (c'est le cas en France dans le cadre de l'UNEDIC), voire par des caisses d'assurance liées aux seuls syndicats (c'est la configuration adoptée par le Danemark et la Suède).
(Finn, 2005, Gratadour, 2005). Il s'agit d'une prise en charge complètement privée des missions de SPE. L'organisation du Job Network en Australie est caractéristique de la deuxième configuration, selon laquelle les fonctions d'intermédiation et d'accompagnement sont soumises à la concurrence entre différents opérateurs, mais la fonction d'indemnisation reste du ressort de l'opérateur public (Considine, 2005). Enfin, la situation des PaysBas est caractéristique de la troisième situation, selon laquelle la fonction d'accompagnement est soumise à un appel d'offres et celle d'intermédiation reste du ressort de l'opérateur public. Ainsi l'instance publique d'indemnisation, $\operatorname{UWV}(5)$, et les municipalités aux Pays-Bas gèrent la procédure d'appel d'offres à des prestataires externes pour la réintégration des demandeurs d'emploi, alors que la fonction de placement direct reste du ressort du secteur public dans le cadre du CWI - Centres For Work and Income - (Delarue, 2005). La situation du Royaume-Uni en dehors des Employment Zones ( $c f$. encadré 3 ) relève aussi de ce schéma, mais l'externalisation des prestations d'accompagnement $\mathrm{y}$ est plus limitée (Struyven, Steurs, 2002).

\section{Encadré 3}

\section{Les Employment Zones}

Ce programme a été introduit en avril 2000 dans quinze zones où le chômage est particulièrement élevé. II recherche une forme d'intervention plus flexible, pour le suivi des publics menacés d'exclusion.

L'accompagnement et l'aide au reclassement des bénéficiaires y sont entièrement confiés à des opérateurs externes en lieu et place des programmes d'accompagnement renforcé pilotés par les Jobcentre Plus, les New Deal. II existe deux types d'Employment Zones; certaines avec un seul opérateur (Working Links, Reed in Partnership, Pertemps Employment Alliance, Work Directions), d'autres avec de multiples opérateurs (1). Dans de telles zones, pour s'assurer que chaque opérateur obtienne une part juste du travail mais aussi pour éviter l'écrémage des demandeurs d'emploi, ceux-ci sont envoyés aléatoirement vers chaque opérateur par les Jobcentre Plus.

(1) L'objectif de l'introduction de multiples opérateurs sur un même marché est non seulement d'analyser l'effet sur la performance globale des employment zones de l'introduction d'une certaine dose de compétition entre opérateurs, mais aussi de laisser le choix à certains clients (parents isolés) de l'opérateur.

(5) Institut pour la gestion des assurances des salariés. 
En France, le choix a été fait jusqu'ici de ne pas complètement ouvrir à la concurrence le marché de l'accompagnement des demandeurs d'emploi, mais au contraire de confier seulement certains éléments de parcours d'accompagnement, d'une durée variant de quelques heures à trois mois, à des opérateurs privés, sur la base d'appel d'offres de marchés publics.

\section{Quelles modalités de recours à des opérateurs privés?}

Le fait de recourir à des opérateurs privés pour réaliser des missions de service public n'est pas propre au SPE. Dans de nombreux secteurs, tels la gestion de l'eau, la distribution d'électricité, les télécommunications ou encore les transports, des opérateurs privés interviennent selon différentes modalités afin de compléter, voire de concurrencer l'offre de l'opérateur public. Cependant, entre la propriété publique et la privatisation totale des biens ou services publics, il existe aussi des procédures intermédiaires : l'externalisation, ou encore la dérégulation partielle, qui met en concurrence l'opérateur public et l'opérateur privé.

\section{L'externalisation de prestations}

L'opérateur public peut externaliser des prestations selon deux options. Dans un premier cas de figure, il peut décider de se désengager sur certains segments de son activité, car d'autres opérateurs sont capables de mieux exercer certaines fonctions. Une telle option se justifie pour de nombreuses raisons (SimONIN, 2004). L'opérateur public peut ne pas avoir les moyens propres de réaliser une action et décider de la confier à un autre organisme. On parle alors d'externalisation de capacité. D'un autre côté, certaines fonctions requièrent une professionnalité spécifique, qui justifie le recours à des prestataires externes. C'est généralement le cas de la formation, surtout professionnelle. En France par exemple, cette fonction est remplie par l'AFPA(6), mais aussi par de nombreux opérateurs privés ou associatifs. Cependant, l'opérateur public peut aussi décider de se dessaisir d'une action, auparavant réalisée par ses services, car il est moins bien placé que d'autres organismes pour assurer sa réussite ou considère que ces organismes peuvent drainer vers lui des "publics cachés», hors de portée des outils communs de la politique d'emploi. Cette configuration est caractéristique de la situation en France dans la mesure où, comme le soulignent BALMARY et alii (2004), la France est le pays d'Europe qui recourt le plus à l'externalisation sous cette forme, en particulier depuis 2001 et la mise en œuvre du Plan d'action personnalisé pour un nouveau départ (PAP-ND). Ainsi, en 2004, les deux tiers des presta-

(6) Association pour la formation professionnelle des adultes. tions d'accompagnement délivrées dans le cadre de ce plan étaient sous-traitées.

Dans la deuxième option, le SPE peut décider de confier à d'autres institutions la responsabilité d'accompagner certains publics. La mise en œuvre des programmes New Deal au Royaume-Uni obéit à ce principe. De même en France, les relations de partenariat établies entre l'ANPE et ses cotraitants (Mission locale, APEC, Cap Emploi) représentent pour l'ANPE une opportunité de s'appuyer sur des structures spécialisées pour accompagner certains groupes de chômeurs.

L'externalisation représente la voie la plus fréquente de recours à des opérateurs privés et c'est cette modalité qui est la plus utilisée en France. Cependant, d'autres pays tels que l'Australie, le Royaume-Uni ou encore les Pays-Bas ont été plus loin en matière de recours à des opérateurs privés. Ils ont en effet instauré des mécanismes de concurrence entre les opérateurs public et privé, dérégulant de fait le marché de l'accompagnement.

\section{L'opérateur public en concurrence avec des prestataires privés}

Introduire des mécanismes de marché pour renforcer la concurrence entre opérateurs public et privé représente une modalité de recours à des opérateurs privés peu utilisée jusqu'à aujourd'hui, mais qui se développe. La Commission européenne (1998) recommandait tout particulièrement que les gouvernements mettent les SPE «quelque peu à l'épreuve du marché ... pour mettre en œuvre une partie des services publics». Les mécanismes de marché les plus usités dans le contexte des SPE sont les appels d'offres dans le cadre de la sous-traitance des services de l'emploi. La dérégulation du service public d'emploi, entendue comme la suppression des restrictions légales à l'introduction de services privés reste l'exception. À titre d'exemple, aux Pays-Bas, l'opérateur public responsable de l'accompagnement des chômeurs a été séparé en deux organismes au début de l'année 2002. D'un côté, le Centre For Work and Income (CWI) qui réalise le premier accueil des chômeurs et établit leur distance présumée au retour à l'emploi. De l'autre, un opérateur public d'accompagnement des demandeurs d'emploi (Kliq) qui est en concurrence avec des opérateurs privés. Et cette concurrence s'est avérée rude : Kliq ne possédait plus que $17 \%$ du marché de l'accompagnement fin 2002, et $3 \%$ fin 2003.

Ainsi, en matière de mise en œuvre des missions de SPE, le choix s'est davantage porté soit sur l'introduction de mécanismes de marché à travers la dérégulation et la création d'appel d'offres (en anglais Tendering), soit sur l'externalisation (en anglais Outsourcing) de certains services à des opérateurs privés, plutôt que la 
privatisation(7) totale des services de l'emploi. D'un point de vue légal ou administratif, l'externalisation et la dérégulation se ressemblent: le service est réalisé par un opérateur externe et la législation concernant les contrats s'applique dans les deux cas. Toutefois, d'importantes différences les séparent. Dans le cadre d'une procédure d'appel d'offres, le gouvernement décide de laisser plus de marge de manœuvre à des fournisseurs externes, tandis que son rôle se limite à des tâches qui ne peuvent être confiées au marché (l'indemnisation, la prise en charge des demandeurs d'emploi) (8).

Le choix des modalités de recours aux opérateurs privés n'est pas neutre sur les relations que ceux-ci entretiennent avec l'opérateur public. En particulier, le recours accru à des prestataires privés, voire la mise en concurrence entre opérateurs public et privé, impliquent de repenser le rôle de l'État comme régulateur du marché de l'accompagnement des chômeurs.

\section{Quels mécanismes de gouvernance mettre en œuvre pour garantir la réalisation des missions de SPE?}

L'objectif de l'introduction d'opérateurs privés est de gagner en efficacité et en efficience, mais aussi en qualité de service. En effet, on s'attend à ce que l'opérateur privé soit en mesure de réaliser le même service que l'opérateur public, à un coût moins important et en s'adaptant mieux aux besoins des usagers du SPE devenus des clients. Cependant, la mission d'accompagnement reste une mission de service public impliquant d'assurer le traitement équitable de chacun, et ce d'autant plus que les besoins des demandeurs d'emploi sont fortement différenciés. Or, cela renforce un risque présent dans toute dérégulation, celui que les opérateurs privés utilisent l'asymétrie d'information en leur faveur et se comportent de manière opportuniste, tandis que le commanditaire public ne peut observer parfaitement les résultats obtenus par ceux-ci.

\section{Les risques associés au recours à des opérateurs privés}

Afin d'augmenter leurs marges, les prestataires privés peuvent privilégier les chômeurs les plus faciles à reclasser. Ils peuvent aussi presser leurs clients d'accepter au plus vite n'importe quel emploi.

(7) Privatisation qui peut se définir comme la vente délibérée par le gouvernement d'entreprises publiques ou d'actifs à des agents économiques privés.

(8) Pour reprendre les termes de Struyven (2004), l'externalisation consiste à contrôler le marché, "control of the market», alors que le processus d'appel d'offre consiste en quelque sorte à être contrôlé par le marché, "control by the market».

\section{Les risques d'aléa moral et de sélection adverse}

Dans le cas de l'accompagnement des chômeurs, l'asymétrie d'information peut engendrer deux modalités de tri des chômeurs par les prestataires.

D'une part, les opérateurs privés peuvent ne sélectionner que les demandeurs d'emploi les plus employables (c'est le risque d'écrémage ou risque d'opportunisme ex ante). D'autre part, une fois les demandeurs d'emploi sélectionnés, ils peuvent décider de concentrer leurs efforts sur ceux qu'ils pensent pouvoir placer facilement, au détriment des autres (c'est le risque de parking ou d'opportunisme ex post). Ces deux types de risque de sélection, «à la porte» ou «après la porte», pour reprendre les termes de Struyven et STEURS (2005) se rattachent aux notions plus générales (9) de sélection adverse (cas où l'information détenue par un contractant est cachée à l'autre, avant la conclusion du contrat) et d'aléa moral (l'action d'un contractant est cachée à l'autre, après la conclusion du contrat). Ils peuvent être utilement analysés dans le cadre de la théorie de l'agence (Jensen, Meckling, 1976).

Cette théorie a été développée dans le cadre de la gestion des entreprises pour formaliser les relations entre actionnaires et dirigeants. Elle vise à comprendre comment un principal, doté d'une certaine fonction objectif, peut amener un ou plusieurs agents ayant leurs intérêts propres, et à qui il délègue une mission à accomplir en son nom, à entreprendre des actions conformes à son objectif. Dans le cadre de cette approche, il s'agit de concevoir des modes d'évaluation de la performance et de trouver des mécanismes incitatifs (notamment à l'aide de contrats) permettant de faire converger les objectifs du principal et ceux des agents.

\section{Le risque de report de la contrainte sur les chômeurs}

Il existe un autre risque lié à l'ouverture à la concurrence de certains services exercés auparavant par le SPE: celui du report de la contrainte subie par les prestataires sur les demandeurs d'emploi.

Des études de terrain réalisées en Belgique, Espagne, France et Royaume-Uni portant sur les liens entre organismes de formation et bénéficiaires de la formation (Darmon, Demazière, Frade, HAAS, 2004) ont mis en exergue certains risques liés à l'introduction de mécanismes de marché dans le domaine de la formation des chômeurs de longue durée, en particulier le fait qu'une «double contrainte» pèse sur les formés et les formateurs. En effet, les organismes de formation se doivent d'accueillir les plus vulnérables des chômeurs, et en même temps d'obtenir de bons résultats en termes de placement. Soumis à des injonctions fortes de la

(9) Notamment utilisées en théorie des assurances. 
part de leurs financeurs, les organismes de formation ne sont pas toujours à même de trouver des «niches» d'emplois permettant un placement aisé de leurs stagiaires. Ils sont alors tentés de résoudre cette contrainte en exerçant une pression sur les demandeurs d'emploi. Ceci peut se manifester de différentes façons. Certains mettant en concurrence les stagiaires sur les mêmes postes afin que ceux-ci agissent comme sur le marché du travail et non comme en formation. D'autres sélectionnent à l'entrée des stages les demandeurs d'emploi les plus motivés. D'autres euphémisent les emplois de mauvaise qualité pour les faire accepter aux stagiaires. Certains organismes de formation vont même jusqu'à donner des primes aux chômeurs qui trouvent rapidement un emploi. Ainsi, dans un contexte de concurrence accrue, les organismes engagés dans la formation des chômeurs de longue durée multiplient les artifices pour atteindre les résultats fixés par les financeurs et finalement transfèrent sur les formés le poids des contraintes qui pèsent sur eux. De tels risques de report de la contrainte ont aussi été repérés au Royaume-Uni dans le cadre des Employment Zones (BRUTTEL, 2004).

Outre les risques précédemment évoqués, d'autres sont apparus dans la pratique, en particulier des disparités locales. En effet, certaines zones, a priori très dynamiques, attirent de nombreux opérateurs, alors que dans d'autres zones, l'offre de service est plus restreinte. D'ailleurs, comme le soulignent BALMARY et alii (2004) dans le cadre de l'externalisation en France, les disparités géographiques sont marquées, et structurées par l'opposition entre les milieux rural et urbain. L'existence de tels risques implique, pour que les missions de service public soient respectées in fine, la mise en oeuvre de mécanismes de gouvernance appropriés (FREYSSINET, 2004), ex ante (assurant la bonne organisation du marché de l'accompagnement) et ex post (des mécanismes d'incitation, d'information et de contrôle). De tels mécanismes visent à faire converger les objectifs des opérateurs publics et ceux des opérateurs privés.

\section{Quels mécanismes de gouvernance?}

Pour prévenir de tels risques, Australiens, Néerlandais et Britanniques ont établi des règles qu'ils int affinées au fil de leur expérience. Quels enseignements en tirer pour le cas français.

\section{Quels mécanismes de gouvernance ex ante?}

Le SPE français se caractérise jusqu'ici par un recours important à des opérateurs externes, mais limité dans son champ à certaines prestations ponctuelles d'accompagnement. Les expérimentations menées par l'UNEDIC et l'ANPE depuis 2005 avec certains opérateurs privés soulignent la volonté de tester de nouvelles modalités d'accompagnement, en donnant davantage d'initiative aux prestataires privés, notamment en leur confiant l'ensemble du parcours d'accompagnement de leur client. Alors que l'implication des opérateurs privés reste au stade de l'expérimentation en France, l'Australie, les Pays-Bas ou encore le Royaume-Uni (dans le cadre des Employment Zones), sont allés plus loin en matière de dérégulation du marché de l'accompagnement. Or, un tel choix s'est accompagné de réformes institutionnelles profondes. En effet, afin de garantir que la concurrence entre opérateurs publics et privés soit effective et viable, les pays qui ont été le plus loin en matière de dérégulation du marché de l'accompagnement, surtout l'Australie et les Pays-Bas, ont repensé radicalement l'organisation du service public de l'emploi. D'une part, ils ont séparé les fonctions de commanditaire de services et celles de fournisseurs. D'autre part, ils ont modifié l'organisation du marché de l'accompagnement pour le rapprocher d'un fonctionnement de quasi-marché (Le Grand, Barlett, 1993).

- La séparation explicite des commanditaires et des fournisseurs de service

L'introduction de mécanismes de concurrence entre opérateurs publics et privés implique au préalable une réorganisation opérationnelle importante, en particulier une redéfinition précise des rôles entre le commanditaire du service (le principal, au sens de la théorie de l'agence) et le fournisseur de service (l'agent, au sens de la théorie de l'agence). Pour de nombreux services publics, l'État est à la fois commanditaire et offreur de service; il décide quel service doit être offert, dans quelle proportion et par qui. Or les évaluations réalisées dans les pays où de tels mécanismes de concurrence existent (Australie, Pays-Bas et Royaume-Uni), suggèrent que le SPE ne peut être en même temps juge et parti, commanditaire et fournisseur. L'exemple de l'Australie est à ce titre très instructif (Considine, 2005). Lors de l'ouverture du marché à la concurrence, l'opérateur public a été scindé en deux, le commanditaire de service (Commonwealth Employment Service (CES)) devenant distinct du fournisseur de service (Employment Assistance Australia (EAA)) sous la supervision de l'autorité de régulation des services de l'emploi (ESRA) (10). Mais du fait de liens étroits entre CES et EAA, le choix des clients entre les différents opérateurs privés était quelque peu biaisé en faveur de EAA. De plus, l'autorité de régulation (ESRA) travaillait dans le même contexte institutionnel, géré par le même ministère qui surveillait étroitement EAA. Des conflits d'intérêt ont commencé à émerger, les règles de parfaite concurrence entre opérateurs n'étaient pas respectées (FAY, 1997, OCDE, 2001). Par la suite, une stricte séparation a été établie

(10) Employment Services Regulatory Authority. 
entre commanditaire et fournisseur de service. Un développement similaire s'est opéré aux Pays-Bas. D'un côté, le CWI est responsable de l'accueil de tous les demandeurs d'emploi et de l'établissement de leur profil. De l'autre, l'opérateur public d'accompagnement des demandeurs d'emploi, Kliq, entre en concurrence avec des opérateurs privés. Une telle séparation entre commanditaire et fournisseur de service en France serait d'autant plus difficile à réaliser que de nombreux acteurs interviennent dans le domaine de l'accompagnement des demandeurs d'emploi, à des niveaux territoriaux différents (MARIMBERT, 2004).

Sur un segment plus limité, celui de la formation qualifiante des demandeurs d'emploi, on a pu expérimenter en France la difficulté, pour un opérateur du SPE lui-même prestataire de formations, d'exercer en même temps la fonction d'orientation et de conseil des demandeurs d'emploi. L'AFPA, qui se trouvait dans cette position lors de la mise en place du PAP-ND, a eu tendance, au moins dans un premier temps, à diriger les chômeurs en priorité vers ses propres stages plutôt que de tenir compte de l'ensemble de l'offre locale (CERC, 2005).

- Créer un quasi-marché apte à accueillir les opérateurs privés (LE GRAND, BARLETT, 1993)

Une deuxième condition préalable à l'instauration de mécanismes de concurrence entre opérateurs publics et privés est la création d'un marché apte à accueillir les opérateurs privés. Le marché de l'accompagnement ne peut être comparé à un marché pur, selon LE GRAND et BARLETT (1993). Il s'agit d'un quasi-marché dans la mesure où les prix sont largement déterminés par la commande publique et le service attendu, fortement lié aux caractéristiques du client, ne peut être optimisé par le simple jeu du marché «ordinaire». Deuxièmement, les ressources publiques sont toujours impliquées, ce qui signifie une surveillance plus étroite de leur affectation. Troisièmement, alors que sur un marché normal, le pouvoir de négociation réside entre les mains du client individuel, dans le cadre du marché de l'accompagnement, ce pouvoir est confié à un acteur qui agit au nom des intérêts des «clients». Enfin, la concurrence n'est pas guidée nécessairement par l'idée de profit car les organismes publics ou privés à but non lucratif qui y opèrent ont des objectifs plus complexes.

Même si le fonctionnement d'un quasi-marché diffère d'un marché normal, les opérateurs privés doivent être incités à rentrer sur ce marché et à concurrencer l'opérateur public (par l'instauration de faibles barrières à l'entrée du marché, des conditions garantissant une concurrence juste et un maximum de transparence sur la constitution des appels d'offres et des contrats).

\section{Quels mécanismes ex post de gouvernance?}

Les mécanismes de gouvernance ex ante ne sont pas suffisants.

Une fois les opérateurs incités à rentrer sur le marché, il faut aussi créer un cadre de régulation (constitution de contrats, incitations financières...) permettant d'éviter les risques précédemment cités (risque d'écrémage, de parking). En particulier, les résultats des différents opérateurs doivent être pilotés (par la fixation d'objectifs de performance et la gestion au résultat). En général, trois types de mécanismes de gouvernance sont utilisés pour résoudre, ou tout au moins réduire, ces problèmes d'aléa moral: des mécanismes d'incitation, d'information et de contrôle (BRUTTEL, 2004, 2005b).

- Les mécanismes d'incitation

L'objectif des mécanismes d'incitation est d'aligner les intérêts de l'agent sur ceux du principal, par la construction de contrats suffisamment motivants.

En premier lieu, l'opérateur public principal a intérêt à constituer des groupes homogènes de clients afin de bénéficier d'une meilleure spécialisation des opérateurs privés. Comment constituer de tels groupes homogènes? Cela implique de trouver des critères objectifs et complets qui permettent de bien identifier les besoins des demandeurs d'emploi. C'est loin d'être évident dans le cas des chômeurs de longue durée, puisque ceux-ci peuvent rencontrer d'importantes difficultés d'ordre professionnel (perte de motivation, de compétences et d'attitudes...) mais aussi relevant d'autres domaines (perte de réseaux sociaux, santé, logement...).

L'Australie et les Pays-Bas, qui ont fortement contractualisé leurs services de l'emploi, ont eu recours à un outil de profilage afin de partager les clients en groupes aux besoins présumés homogènes : le Jobseeker Classification Instrument en Australie et le Kansmeter aux Pays Bas. Le profilage permet, dans un premier temps, de classer chaque personne dans une catégorie définie en fonction de critères objectifs (âge, sexe, diplôme...). Dans un deuxième temps, le profilage joue un rôle déterminant lors de l'accompagnement vers l'emploi car il permet aux SPE de sélectionner les personnes les plus difficiles à reclasser sur lesquelles il est nécessaire de concentrer les moyens (CAhuc, Kramarz, 2004). Ainsi, aux Pays-Bas, le profilage sert à orienter les chômeurs. Ceux qui sont jugés les plus faciles à placer restent suivis par le CWI, ceux auxquels on offre un accompagnement plus soutenu sont orientés vers la caisse d'assurance chômage (s'ils sont indemnisés) ou la commune (s'ils bénéficient de l'aide sociale), qui font appel aux prestataires privés, en totalité dans le premier cas, à hauteur de $70 \%$ pour les communes.

Soulignons qu'il est important que ce soit une autorité indépendante des opérateurs et des financeurs des allocations, comme c'est le cas de CWI 
aux Pays-Bas, qui réalise le profilage et l'orientation des demandeurs d'emploi.

En France, dans le cadre des expérimentations actuelles, l'ANPE et l'UNEDIC ont aussi recours à cette technique de profilage en partie appuyée sur un outil statistique pour sélectionner les demandeurs d'emploi qui ont un fort risque de chômage de longue durée. La détermination du profil permet non seulement d'orienter les demandeurs d'emploi vers les services les plus adaptés à leurs besoins et leurs difficultés, mais aussi de déterminer les résultats à atteindre et la structure des paiements liés à de tels résultats pour chaque groupe homogène de clients.

En préalable à toute contractualisation, le type de service attendu par le SPE doit être précisément défini, de même que le résultat attendu pour chaque groupe de clients (CAHUC, KRAMARz, 2004). Le résultat privilégié indique la priorité fixée par l'opérateur public principal. En particulier, la sortie du chômage vers une formation représente-t-elle un résultat en soi ou est-ce que seules les sorties vers l'emploi peuvent être considérées comme des résultats? La durée de l'emploi retrouvé doit-elle être prise en compte?

Dans le cas néerlandais, seules les transitions vers l'emploi sont comptabilisées. Elles sont différenciées en fonction de la durée de l'emploi retrouvé. Comme le soulignent Bruttel et Sol (2006), dans les trois pays où l'instauration de mécanismes de concurrence sur le marché de l'accompagnement est la plus étendue (Australie, Pays-Bas et RoyaumeUni), le choix s'est porté sur des indicateurs en termes d'emploi, ce qui reflète une philosophie de type Work First. Cela n'empêche pas que des arbitrages différents soient possibles.

Déterminer le prix associé à l'appel d'offres et la structure de paiement représente un deuxième élément clé dans la structuration des contrats. Il est lui aussi très difficile à déterminer, dans la mesure où il n'existe pas de prix de marché évident. La structure de paiement choisie par le principal doit inciter l'agent à respecter ses engagements, et en particulier à privilégier un placement «durable» en emploi, tout en lui permettant d'être rentable. Notons que d'ailleurs la notion de durabilité souhaitable de l'emploi varie fortement d'un pays à l'autre. Ainsi, les contrats d'accompagnement des demandeurs d'emploi comprennent généralement une part fixe versée à l'opérateur quel que soit le résultat atteint et une part variable attribuée uniquement si un emploi est obtenu et plus particulièrement un emploi stable. Un mécanisme similaire avait été décrit dans le cas du quasi-marché de la formation des chômeurs (Stankiewicz, 1999).

Dans le cadre des expérimentations menées en France, le choix a été fait de créer des contrats en différenciant la structure des paiements en fonction de l'âge du demandeur d'emploi accompagné (moins de 50 ans et plus de 50 ans) et de la durée de l'emploi retrouvé (moins ou plus de 182 jours).

\section{- Les mécanismes d'information}

La mise en œuvre de mécanismes d'incitation a pour but de faire converger les objectifs de l'agent avec ceux du principal, tandis que l'instauration de mécanismes d'information vise à réduire l'asymétrie d'information. Pour ce faire, le principal recueille des informations sur les efforts fournis par les prestataires, généralement en comparant leurs performances respectives, par des techniques dites de Benchmarking. L'Australie a d'ailleurs introduit un système très sophistiqué, le Star Rating qui compare les résultats de placement de tous les opérateurs présents sur le marché en utilisant des modèles de régression (du type logit/probit) contrôlant pour les conditions du marché du travail local et les caractéristiques des demandeurs d'emploi. En fonction des résultats obtenus, le SPE attribue des étoiles à chaque opérateur. Ce système est très incitatif dans la mesure où les opérateurs classés dans les deux premiers tiers pourront obtenir des contrats lors de l'appel d'offres suivant sans concourir (BRUTTEL, 2005a). Aux PaysBas, le choix s'est porté sur l'instauration d'un label de qualité Borean Reintegration Quality Mark, et sur la diffusion, tous les six mois, d'un rapport sur la qualité des différents prestataires (Delarue, 2005). Il convient de préciser que la réputation des firmes dans ce marché peut aussi servir de mécanisme important de régulation de marché si l'information sur les différents opérateurs est largement diffusée, comme c'est le cas aux Pays-Bas et au Danemark.

- Les mécanismes de contrôle

Enfin, des mécanismes de contrôle plus traditionnels, appuyés sur des règles, ont été mis en place. Il s'agit par exemple du code des pratiques des services de l'emploi en Australie ou du Guide de l'opérateur des Employment Zones au RoyaumeUni (BRUTtEL, 2005b).

$$
\text { * } *
$$

L'analyse de pays étrangers parmi les plus actifs dans l'implication d'opérateurs privés dans leur système d'intervention sur le marché du travail (l'Australie, les Pays-Bas, le Royaume-Uni) éclaire, à notre avis, la situation française actuelle.

En effet, dans aucun de ces pays, la dérégulation du marché de l'accompagnement et l'instauration de certains mécanismes de concurrence entre opérateurs publics et privés ne se sont traduits par un désengagement de l'État. Bien au contraire. Ce qui frappe, comme le souligne SimONIN (2004), c'est la volonté d'inscrire des initiatives privées dans le cadre d'une véritable stratégie d'animation et de pilotage du marché du travail de la part de l'opérateur public. 
Grâce à des réformes radicales, le rôle de chaque opérateur a été clarifié, notamment aux Pays-Bas.

Ce sont les pouvoirs publics nationaux qui tracent les contours du système, répartissent les rôles entre les différents acteurs publics et privés, leur fixent des objectifs opérationnels. De fait, les pouvoirs publics, dans les pays étudiés (si on se limite aux Employment Zones pour le Royaume-Uni) s'apparentent plutôt à un régulateur qu'à un opérateur particulier du système. Le rôle régulateur de pouvoirs publics pour les opérations sous-traitées, plutôt que celui d'opérateur direct, ne vide pas pour autant le Service public de l'emploi, en tant qu'institution, de son contenu. En effet, de nombreuses missions de service public ne peuvent être confiées efficacement à des opérateurs privés et doivent continuer de relever de l'opérateur public. C'est le cas de l'inscription au chômage, de la gestion des allocations (au moins pour la partie qui n'est pas gérée par les partenaires sociaux), du contrôle de la recherche d'emploi, de la gestion du système d'information sur le marché du travail - recueil des offres d'emploi vacantes, appariement et diffusion de l'information. C'est évidemment le cas aussi de la sélection des opérateurs, du suivi et de l'évaluation de leurs activités (Simonin, 2004). Même les pays les plus en faveur de la dérégulation du service public de l'emploi ont du faire marche arrière sur ce point.
À titre d'exemple, en Australie, la fonction de recueil des offres d'emploi vacantes a été ouverte à la concurrence. Cette ouverture s'est avérée fortement contre-productive (Considine, 2005, OCDE, 2001). D'ailleurs, l'étude menée par FougĖre et alii (2005) souligne que les coûts de recherche d'emploi sont réduits grâce à l'intervention d'une agence publique qui centralise l'ensemble de l'information.

Plutôt que d'être appréhendé comme une menace, le recours à des opérateurs privés peut être vu comme une opportunité pour l'opérateur public de partager certaines de ses responsabilités en matière d'accompagnement des chômeurs, tout en maîtrisant le cadre de régulation général du marché de l'accompagnement pour garantir le respect des missions de service public.

En France, ce peut être une chance intéressante à saisir pour au moins deux raisons. D'une part, le taux de chômage reste depuis des années à des niveaux importants, en particulier pour les jeunes, les travailleurs seniors et les personnes faiblement qualifiées. D'autre part, l'ANPE doit maintenant gérer aussi des flux plus importants de chômeurs, les entretiens étant mensuels à partir du quatrième mois de chômage. Dans ces conditions, il lui sera difficile d'offrir sur ses propres ressources un accompagnement personnalisé et intensif à des demandeurs d'emploi rencontrant des difficultés particulièrement tenaces sur le marché du travail.

\section{Bibliographie}

Balmary D., Chevrier-Fatome C., Simonin B. (2004), Rapport de l'instance d'évaluation de la politique d'emploi et recours à des opérateurs externes, La Documentation française, Paris.

Barbier J.-P., Hansen E., Samorodov A. (2003), "PublicPrivate Partnerships in Employment Services", Working Paper $\mathrm{N}^{\circ}$ 17, BIT, Genève.

Berthet T. (2006), L'État social à l'épreuve de l'action territoriale: Postmodernité et politiques publiques de proximité dans le champ de la relation formation-emploi, journées d'études de l'Association française de science politique, IEP de Grenoble, 15 et 16 juin.

Bruttel O. (2004), "Contracting-Out the Public Employment Service and the consequence for Hardto-Place jobseekers: experiences from Australia, the Netherlands and the UK", Paper for the Second Annual ESPAnet Conference, Oxford, 9-11 September.

BRuTTEL O. (2005 a), "Managing competition in a public service market: the Job network in an international Perspective", CLMR Discussion Paper.
Bruttel O. (2005 b), “Contracting-Out and Governance Mechanisms in the Public Employment Service", WZB Discussion paper.

Bruttel O., Sol E. (2006), "Work First as an European Model? Evidence from Germany and the Netherlands", Policy and Politics, 34, à paraître.

Cahuc P., Kramarz F. (2005), De la précarité à la mobilité: vers une Sécurité sociale professionnelle, rapport au ministre d'État, ministre de l'Économie, des Finances et de l'Industrie et au ministre de l'Emploi, du Travail et de la Cohésion sociale, Paris, La Documentation française.

Cerc (2005), Aider au retour à l'emploi, rapport $n^{\circ} 6$, Paris, La Documentation française.

Commission EuRopéEnNe (1998), Moderniser le SPE pour soutenir la stratégie européenne pour l'emploi, communication du 13 novembre.

Considine M. (2005), "The reform that never ends: quasi-markets and employment services in Australia", in Contractualism in Employment Services. A new form of 
welfare state governance, E. Sol et M. Westveld eds, L. Kluwer Law International, La Haye.

Cour Des Comptes (2006), L'évolution de l'assurance chômage: de l'indemnisation à l'aide au retour à l'emploi, rapport public thématique.

Darmon I., Demazière D., Frade C., HaAs I. (2004), «Formés et Formateurs face à la « double contrainte» des programmes de formation à l'employabilité des chômeurs de longue durée», Formation Emploi, n ${ }^{\circ} 85$.

Delarue V. (2005), «Politiques et institutions néerlandaises d'aide au retour à l'emploi», Les Papiers du CERC, $\mathrm{n}^{\circ}$ 2005-03, novembre.

De Koning J., Denays J., Walwei U. (1999), “Deregulation in Placement Services: a comparative study for eight EU countries", Report for the European Commission.

DEPARTMENT FOR WORK AND PENSIONS (2005), "The use of contestability and flexibility in the delivery of welfare services in Australia and the Netherlands", Research Report, $\mathrm{N}^{\circ} 288$.

FAY R.G. (1997), "Making the Public Employment Service more effective through the introduction of market signals", Labour Market and Social Policy Occasional Papers $\mathrm{n}^{\circ} 25$, OECD, Paris.

FINN D. (2005), "The Role of contracts and the private sector in delivering Britain's 'Employment First' Welfare State", in Contractualism in Employment Services. A new form of welfare state governance, E. Sol et M. Westveld eds, L. Kluwer Law International, La Haye.

Fougère D., Pradel J., Roger M. (2005), “Does JobSearch Assistance affect effort and Outcomes? A Microeconometric Analysis of Public versus Private Search Methods", IZA Discussion Paper Series.

Freyssinet J. (2004), «Politique de l'emploi: les conditions de la Gouvernance», Connaissance de l'Emploi.

Gratadour C. (2005), «Politiques et institutions britanniques d'aide au retour à l'emploi», Les Papiers $d u$ CERC, $\mathrm{n}^{\circ}$ 2005-05, novembre.

Herbillon J.-M. (2004), «Le "Profiling” des demandeurs d'emploi aux Pays-Bas », Travail et Emploi, n 99, juillet, Ministère du Travail et de la Cohésion Sociale, Paris.

Hootganders Y., E. Sol (2005), “Steering by Contract in the Netherlands : New Approaches to Labour Market Integration", in Contractualism in Employment Services. A new form of welfare state governance, E. Sol et M. Westveld eds, L. Kluwer Law International, La Haye, 2005.

Jensen M., Meckling W. (1976), "Theory of the firm, managerial behaviour, agency costs and ownership structure", Journal of Financial Economics, 13, pp. 305-340.

Joly B. (2005), «Politiques de l'emploi en Europe: les grandes tendances chez les voisins de la France», Droit Social, $\mathrm{n}^{\circ} 1$, janvier.

Le Grand J., Bartlett W. (1993), Quasi-Markets and Social Policy, London, MacMillan Press.

MARIMBERT J., JOLY B. (2004), Le rapprochement des services de l'emploi, rapport au ministre des Affaires sociales, du Travail et de la Solidarité, Paris, La Documentation Française.

OCDE (2001), Des politiques du marché du travail novatrices : la méthode australienne.

Rousseau Y. (2005), «Du monopole public du placement à un nouveau service public de l'emploi», Droit Social, $\mathrm{n}^{\circ} 4$, pp. 456-465.

Simonin B. (2004), «Politique de l'emploi : trois réformes à l'étranger», Connaissance de l'Emploi, $\mathrm{n}^{\circ} 3$, mai.

Stankiewicz F. (1999), «L'efficacité de la formation délivrée aux chômeurs. L'État et la solvabilisation de la formation», Revue Économique, volume 50, $\mathrm{n}^{\circ}$ 2, pp. 273-290.

Struyven L. (2004), "Design Choices in Market Competition for Employment Services for the Longterm Unemployed", Social, Employment and Migration Working Papers, $\mathrm{n}^{\circ} 21$, OECD, Paris.

Struyven L., Steurs G. (2002), “The competitive Market for Employment Services in the Netherlands", Social, Employment and Migration Working Papers, $\mathrm{n}^{\circ} 13$, OECD, Paris.

Struyven L., Steurs G. (2005), "The creation of a quasi market for the reintegration of jobseekers: Empirical evidence from Australia and the Netherlands", Journal of European Social Policy, Volume 15, pp. 211-229. 


\section{Annexe \\ Les différents systèmes de services publics de l'emploi \\ en Australie, aux Pays-Bas et en Grande-Bretagne}

\section{Quelles réformes du Service Public de l'Emploi en Australie?}

Le système australien est un système fédéral, mais les municipalités et les provinces n'ont qu'un rôle très limité dans l'offre des services d'emploi et dans la gestion des revenus de remplacement des chômeurs. L'essentiel du pouvoir de décision est détenu par l'État. Avant 1994, l'ancien opérateur public Commonwealth Employment Service (aujourd'hui remplacé par Centrelink) contractualisait seulement la formation et les clubs de recherche d'emploi à des opérateurs privés. Depuis, ce système n'a cessé d'être modifié. En particulier, la réforme Working Nation du gouvernement travailliste a mis en place en 1994 un système où le tiers des services de réintégration était contractualisé à des agences à but lucratif et non lucratif. L'arrivée du Gouvernement conservateur de John Howard en mars 1996 donna une nouvelle impulsion, en réformant en profondeur l'agence publique et en renforçant l'implication du secteur privé. Celui-ci intervenait non seulement en matière d'accompagnement des chômeurs, mais aussi de recueil des offres et des demandes d'emploi (fonction d'appariement). Cette situation étant sous optimale (confusion chez les demandeurs d'emploi, concurrence entre opérateurs pour obtenir des offres d'emploi vacantes), de nouvelles réformes ont été menées depuis, en mai 1998 (1 er $^{\text {Job Network), en }}$ février 2000 (2e Job Network) et surtout en mai 2002 (3e Job Network).

La succession de ces réformes a remodelé la structure du quasi-marché de l'accompagnement des chômeurs. En particulier, les opérateurs présents sur le marché et les relations établies entre le Service Public et les opérateurs privés se sont transformés. En effet, depuis le 1er Appel d'Offre du Job Network, le nombre d'opérateurs s'est considérablement réduit, et la part du secteur public a fortement décliné. En 1998, 310 opérateurs se faisaient concurrence $(33 \%$ du secteur privé à but lucratif, $30 \%$ du secteur associatif, $37 \%$ du secteur public) contre 197 en 2000 (47\% privés, 45\% associatifs, $8 \%$ publics). En 2002, le marché s'est nettement concentré avec 109 opérateurs présents; les 5 premiers détenant $39 \%$ des parts de marché et les 10 premiers $55 \%$ (BRutTel, 2005a, Considine, 2005). La place désormais résiduelle de l'opérateur public a incité l'État à se concentrer sur son rôle de régulateur du système dans son ensemble. À ce titre, la sélection des opérateurs du Job Network revient à Centrelink qui dépend de deux ministères. Le contrôle des opérateurs et celui des appels d'offre passés est assuré par deux organismes: un contrôleur indépendant (Probity Advisor) et un office national de vérification des comptes (Australian National Audit Office).

\section{Quelles réformes du Service Public de l'Emploi aux Pays-Bas?}

Aux Pays-Bas, la décentralisation du Service Public de l'Emploi (SPE) et la plus forte implication de sous-traitants privés ont été introduits pour améliorer la qualité et l'efficience du service offert aux demandeurs d'emploi. Cette politique d'implication croissante des autorités locales et des opérateurs privés s'est effectuée graduellement (Delarue, 2005). De 1990 à 1995, le SPE a été décentralisé. De 1996 à 1999, le pilotage d'ensemble du SPE et le financement des activités d'aide au retour à l'emploi ont été profondément modifiés. Pour une frange de son activité, l'opérateur public est devenu prestataire de services pour les instances versant les allocations (la caisse d'assurance chômage UWV et les communes). Cette période correspond à une période de transition vers le marché. Enfin, en 2000, les instances versant les allocations ont eu la possibilité de choisir librement leurs sous-traitants pour l'ensemble des opérations en lien avec l'aide au retour à l'emploi.

Avec la mise en œuvre de la loi SUWI en 2002, une dernière étape est franchie. L'Office Central pour l'Emploi a été scindé en deux entités. L'ensemble des activités publiques d'accompagnement vers l'emploi a été transféré à la société commerciale Kliq, dont l'État reste le principal actionnaire. La 2e institution, le: Centre For Work and Income (CWI), a un rôle de guichet unique. Elle accueille les demandeurs d'emploi, établit leur profil et les oriente vers UWV si le demandeur d'emploi est éligible à l'assurance chômage ou vers les municipalités, s'il est bénéficiaire de revenus d'assistance. UWV et les municipalités contractent directement avec les opérateurs privés. Les parts de marché de Kliq ont suivi la même évolution que celles de l'ancien opérateur australien. Elles ont rapidement chuté, de $17 \%$ en 2002 à moins de $3 \%$ en 2003. En revanche, contrairement au marché australien où les associations jouent un rôle important, ce sont les entreprises de travail temporaires qui sont le plus présentes (BRUTTEL, 2005a). 


\section{Quelles réformes du Service Public de l'Emploi au Royaume-Uni ?}

Les réformes actuelles des services de l'emploi britanniques renforcent le rôle de l'acteur public. Toutefois, en parallèle, le recours aux opérateurs du secteur privé et bénévole (organismes à but non lucratif) s'accentue aussi (BALMARY, 2004). Sur le plan opérationnel, les Jobcentre Plus, guichets uniques d'accueil, sont au cœur du système britannique d'aide au retour à l'emploi tandis que les décisions stratégiques et le pilotage du système sont du ressort du Department For Work and Pensions. Ces organismes ont une politique de partenariat très développée, qui concerne des institutions publiques (Neighbourhood Renewal Unit et Social Exclusion Unit) et les organismes privés opérant dans le domaine de la formation (les Learning and Skill Councils) et de l'accompagnement des chômeurs.

Avant 1997, peu d'agences privées jouaient un rôle significatif dans le développement des programmes de politique de l'emploi. Ensuite, le gouvernement du New Labour a décidé de confier au secteur privé une partie de la mise en œuvre du programme New Deal for Young Unemployed People dans 2 des 144 zones. Depuis, de plus en plus d'opérateurs privés ont été impliqués et finalement $10 \%$ des dispositifs New Deal sont maintenant réalisés par des opérateurs privés (FInN, 2005). La création des dispositifs Employment Zones et Action Team For Jobs a encore renforcé l'implication des opérateurs privés dans l'accompagnement des demandeurs d'emploi, en particulier celui des plus éloignés du marché du travail (Herbillon, 2004). Finalement, les Jobcentre Plus accompagnent vers l'emploi les demandeurs les plus faciles à placer et une partie seulement des plus défavorisés. Dans les Employment Zones, les opérateurs privés suivent l'ensemble du parcours du demandeur d'emploi (Gratadour, 2005), alors qu'ailleurs, ils effectuent des prestations plus ponctuelles. 


\section{Le capital social, performance, équité et réciprocité}

\section{Sous la direction d'Antoine Bevort et Michel Lallement}

Éditions la Découverte, 2006

\section{Lu par Marie Wierink(*)}

Début 2006, les Éditions la Découverte publient un ouvrage collectif consacré au «capital social», sous la coordination d'Antoine Bevort et Michel Lallement. Cet ouvrage vient utilement éclairer un concept qui a curieusement encore peu de retentissement dans les travaux sociologiques français, en comparaison de son rayonnement scientifique à l'étranger ou dans les organisations internationales. Issu des travaux du politologue Robert Putnam, le concept de «capital social» rend compte de l'effet du dynamisme associatif d'une société, de la capacité de ses membres et de la société civile à mener des initiatives ou des actions communes, de la vitalité de la démocratie et du bon fonctionnement de ses institutions. «Par rapport aux approches habituelles de la socio-économie (et notamment celles qui sous-tendent les théories du bien-être et les indicateurs sociaux), le capital social introduit la dimension holistique du lien entre les personnes » (p. 296, contribution de B. Perret). Cependant, audelà de cette définition, la notion de capital social est extensive - c'est d'ailleurs une de ses faiblesses - et au fil de la lecture de l'ouvrage, il semble qu'il faille accepter cette plasticité pour en reconnaître toute la dimension heuristique. Le grand mérite de ce recueil est de mettre en évidence la genèse du concept et ses parentés multiples avec d'autres approches théoriques tant politicologiques que sociologiques; il en démontre le caractère fécond pour une approche «compréhensive», macrosociologique et dynamique de l'évolution des sociétés, aussi bien que ses limites théoriques et pratiques, et souligne les possibilités multiples, même si elles sont ardues, de mobilisation de cette théorie au plan microsociologique.

L'ouvrage est composé de trois parties. La première, à laquelle nous rattacherons les éclairantes préface et introduction d'Alain Caillé, Antoine Bevort et Michel Lallement, donne les clefs de lecture du concept et en retrace la généalogie, tout en en présentant une analyse rigoureuse et critique sous la plume de S. Ponthieux. La seconde partie présente les résultats de travaux de sociologie quantitative visant à mesurer les effets de variables construites à partir de la théorie du capital social, dans des champs aussi divers que la société malgache, la sociologie de l'éducation ou les rela-

(*) Mission animation de la recherche, DARES. tions professionnelles. Enfin, la troisième partie réunit des articles centrés sur l'étude des liens entre capital social et formes d'action collective, telles que les associations ou la petite entreprise familiale créée en France par des immigrés maghrébins. L'ouvrage se termine par une conclusion de JeanBaptiste de Foucault ouvrant sur des perspectives opérationnelles: le capital social peut constituer un «macroscope», selon l'expression de l'auteur, pour analyser l'évolution du rapport des individus aux institutions, aux politiques publiques et à l'exclusion, et «éclairer l'action».

\section{Une notion extensive ...}

La première partie dresse les contours du concept, en retrace l'histoire et le soumet à la critique. Peuton, sans risquer le paradoxe, utiliser une notion telle que «capital» social pour critiquer l'utilitarisme et l'étroitesse du raisonnement économique? Comment passer du «capital humain» à la Becker au «capital social» de Putnam et ses prédécesseurs, Coleman et Burt? Comment démêler dans le «capital social» ce qui relève d'un capital individuel, celui d'un individu au mieux inséré dans des réseaux, et ce qui relève du capital collectif d'une société, symbolisé par la confiance et la réciprocité des échanges qui y règnent? La première partie ouvre sur deux textes fondateurs de la diffusion du concept en France, écrits par Pierre Bourdieu et Robert Putnam. On a là un curieux effet de miroir entre deux approches d'une même intuition, l'une insistant sur les effets de concentration du capital social et de domination qu'il permet d'exercer, l'autre sur les effets de confiance ou de réciprocité. Dans le texte de Pierre Bourdieu, publié comme "Notes provisoires» dans Actes de la recherche en Sciences sociales en 1980, le capital social apparait comme un prolongement, un «multiplicateur» des capitaux économiques et culturels et de leurs effets sur la place des individus et sur les rapports de domination dans le système social. Le texte de R. Putnam, au titre évocateur, Bowling alone (1995) est également connu. Après avoir travaillé sur la société italienne et ses contrastes entre le Nord et le Sud, encore dominé par des solidarités étroitement familiales, Putnam analyse la déperdition en capital social de la société américaine. Il tire ce constat de la diminution de la participation à la vie politique et civique, que Putnam associe à celle de l'associativité et de la confiance réciproque; il en souligne les rapports avec des évolutions sociales de fond comme la mobilité géographique intensifiée, la pression temporelle liée à l'activité féminine, le changement démographique, les réorganisations urbanistiques et l'individualisation des loisirs allant de pair avec les nouvelles technologies de communication. Comme le montre M. Lallement dans un passionnant chapitre où il en décortique les filiations et les proximités, la théorie du capital social apparaît ainsi intimement liée aux grands courants de la sociologie économique, mais trouve aussi des enracinements dans des travaux de nature culturaliste, tels que ceux de Tocqueville et de Max Weber. 
On y verra des liens évidents avec les notions d' $\mathrm{em}$ beddedness, d'encastrement et la théorie des liens faibles de Mark Granovetter, ou avec la théorie des réseaux. Mais le capital social fait aussi écho aux débats qu'animent des sociologues ou politologues américains aussi différents que Richard Sennett, critique de la flexibilisation du travail et de l'individualisation de la société américaine, ou que Francis Fukuyama, qui voit dans les sociétés à haut degré de confiance les sociétés les mieux armées pour la performance économique. Selon M. Lallement, trois dimensions principales organisent les diverses conceptions du capital social: la confiance, les réseaux ou la réciprocité. La plasticité de la notion renvoie à des usages bien différents : un souci économique de démontrer que le capital social conduit à la performance économique, un souci politique visant à démontrer l'amélioration de la vie démocratique, ou encore une préoccupation sociale visant à décortiquer les ressorts de certaines inégalités.

\section{... souffrant d'une certaine imprécision}

C'est bien à partir de cette plasticité et de ce côté «concept valise» que S. Ponthieux, économiste, organise une critique stimulante du «capital social». En définitive, n'y aurait-il pas imposture à présenter comme une nouvelle clé de lecture de l'évolution des sociétés un ensemble de phénomènes dont l'importance est reconnue depuis longtemps par les économistes? Sophie Ponthieux analyse de manière méthodique la dénomination même de «capital social», en insistant sur le flou qui l'entoure: doit-on entendre ce capital comme un facteur de production au sens économique du terme ou comme une ressource, dans une acception plus générique? Cela lui permet de contraster le recours fait à ce concept par P. Bourdieu, chez qui elle reconnaît un usage plus économique du terme de «capital social», et le «social capital» de R. Putnam, dans le sillage des travaux de J. Coleman, qui tend à «bâtir un pont théorique entre la conception "sursocialisée" de l'acteur des sociologues et la conception «sous-socialisée » de l'agent rationnel des économistes ». C'est alors l'imprécision même du social capital qui rend sa mesure complexe, les multiples variables qu'il est possible de retenir renvoyant à des dimensions différentes, qu'il s'agisse de pratiques, d'opinions, de perceptions, sans qu'il soit sérieux, pour S. Ponthieux, d'en tirer des conclusions solides en termes de causalité. L'auteur a alors une formule lapidaire: et si le social capital n'était autre que le résidu sur lequel achoppent bien des travaux économétriques? La critique de S. Ponthieux se fait alors plus politique: l'intérêt des institutions internationales pour les études mobilisant le social capital ne renverrait-il pas à l'émergence de compromis politiques du type troisième voie, détournant des analyses plus traditionnelles faites en termes d'inégalités et de dominations?

\section{Capital social et pratiques d'entraide}

La seconde partie de l'ouvrage sort de ces débats théoriques et présente les résultats de travaux inspirés de l'approche du capital social. Un cadre théorique qui voit dans les relations de coopération et de confiance entre les individus une «ressource» est évidemment attractif pour construire une analyse des pratiques d'entraide quotidienne développées à l'intérieur du voisinage ou de la parentèle, et pour examiner si la mobilisation de «réseaux » est à même de compenser d'autres inégalités économiques et sociales. La première étude est consacrée à un tel projet (A. Degenne, M.-O. Lebeaux, et Y. Lemel). Elle a été basée sur une exploitation secondaire de l'enquête Modes de vie de l'INSEE qui recensait sur la période 1988-1989 des informations sur les ressources financières, les budgets temps et les aides et relations familiales de près de 7000 ménages. Une des conclusions est bien que «l'apport des réseaux ... contribue bien à l'amélioration du bienêtre des plus jeunes et des plus âgés ». La seconde contribution s'intéresse à l'effet du capital social d'un point de vue plus macrosocial: il s'agit de tester économétriquement si l'intégration plus ou moins forte des ménages dans la structure sociale, mesurée par leur participation à différentes formes d'organisation collective de la vie rurale dans un territoire, va de pair avec l'amélioration relative de leurs conditions de vie, mesurée par le montant de leur consommation par tête. Là encore, une relation de corrélation est bien constatée.

\section{Deux champs d'application: l'école et l'entreprise}

La sociologie de l'éducation constitue un autre terrain où depuis longtemps sont décelés les effets sur la performance scolaire de la détention de différentes ressources en capital humain, culturel, et économique par les parents. Trois contributions vont s'intéresser à ce champ. Une équipe de chercheurs suisses (Favre et Jaeggi) va s'intéresser aux effets du capital social sur la réussite des jeunes enfants de quartiers défavorisés, en dépassant cette approche individuelle pour questionner l'influence de l'attitude des parents à l'égard de la sociabilité enfantine et de l'établissement de relations d'échanges avec les enseignants et autres personnels de l'école. Leurs travaux conduisent à des résultats en demiteinte: il faut ajouter à l'analyse la capacité des parents à s'inscrire dans la «culture» scolaire, et à ce titre à dépasser une conception seulement «utilitaire» des relations concrètes entre l'école et la famille. Antoine Bevort et Danielle Trancart aborde la question scolaire sous un autre angle, celle des inégalités territoriales en matière d'éducation, à la manière dont R. Putnam dans ses travaux sur l'Italie testait la performance institutionnelle de l'Italie du Nord et du Sud à partir des formes et de la densité des relations associatives et de réciprocité. Les auteurs vont mener une analyse factorielle de correspondances à partir de données tirées de l'enquête Conditions de vie des ménages et croiser les variables individuelles de formation, revenus, participation à la vie associative et la situation géographique, puis croiser le classement des régions obtenues avec les performances scolaires de ces régions. Si l'exer- 
cice économétrique est complexe et si les données restent fragiles et agrégées à des niveaux (les académies) dont on peut discuter la pertinence, il en ressort toutefois que le lien entre les inégalités économiques et les performances scolaires est redoublé par celui entre ces inégalités et la distribution du capital social. Pour ces auteurs, la problématique du capital social est féconde dans l'analyse des difficultés des politiques scolaires à assurer la démocratisation de l'école, mais ils s'accordent avec l'équipe suisse pour reconnaître l'importance de la construction du «sens » accordé à l'école chez les populations défavorisées. Enfin, la troisième contribution portant sur l'éducation s'intéresse à l'insertion professionnelle de diplômés de troisième cycle en gestion des institutions culturelles participant à différentes unités de formation. L'hypothèse est que le capital social, développé sous la forme d'un "système d'action» impliquant des étudiants mobilisés autour de l'insertion, des employeurs à la recherche de candidats et une équipe pédagogique, sera déterminant. Selon que les dispositifs de formation offrent un capital social plus ou moins important, sous la forme d'une articulation école/monde du travail plus ou moins serrée, les résultats en termes d'insertion professionnelle dans un premier emploi seront plus ou moins favorables. Ce capital social «éducatif» peut même, selon l'auteur, compenser les inégalités individuelles en termes de capital social d'origine familiale ou d'expérience antérieure.

L'entreprise, vue comme un «monde social», constitue un autre champ où une analyse en termes de capital social ouvre des possibilités nouvelles, en permettant de l'interroger comme «forme sociale». L'ambition d'Antoine Bevort, dans cette contribution, est de montrer que le capital social constitue aussi un instrument microsociologique pour étudier l'entreprise. À partir des données de l'enquête Réponse de la DARES, l'auteur teste l'influence du capital social sur les pratiques innovantes de gestion ou d'organisation du travail, à partir des variables décrivant l'intensité des relations sociales, le climat social et l'implication des acteurs des relations professionnelles dans leurs réseaux organisationnels respectifs syndicaux et patronaux. Si l'effet taille joue sur les pratiques innovantes dans les grandes entreprises, en revanche, pour Antoine Bevort, le capital social ainsi défini semble bien, au moins indirectement, influent dans l'aire des entreprises de moins de 200 personnes.

\section{Capital social et action collective}

Enfin, la troisième partie s'intéresse à la manière dont capital social et action collective sont liés. La première contribution (E. Lazega), se place sur un terrain théorique. Elle plaide pour que la sociologie et la modélisation n'abandonne pas aux marketers et aux administrations publiques le terrain de l'exploitation secondaire des fichiers divers retraçant les affiliations multiples des individus; ils constituent une formidable base de données méso-sociale «des interdépendances économiques et sociales entre acteurs individuels et/ou organisationnels», enrichissant la connaissance des « réseaux complets de relations entre participants». La seconde (J.-P. Worms), plus descriptive, fournit quelques clés de lecture de l'évolution de l'univers associatif. Elle commence par donner quelques repères historiques du dynamisme de création des associations, mais montre aussi l'évolution de la nature de celles-ci, parmi lesquelles la part des associations sportives et culturelles recule au profit tout d'abord d'associations d'intérêts catégoriels (décennie 70) puis de l'apparition d'engagements altruistes, et enfin d'associations à vocation sociale au fur et à mesure du désengagement de l'État-providence. Les formes d'engagement associatif évoluent aussi au profit d'engagements à tonalité plus individuelle que collective, dans une relation plus coopérative qu'identitaire, et dans un zapping très éloigné de l'engagement associatif traditionnel, difficile à gérer par les responsables. Changement d'époque, changement de modèle, les avantages de la visibilité et de la stabilité de l'ancien modèle associatif disparaissent au profit d'une plus grande sensibilité associative aux courants sociaux émergents, dans ce que l'auteur appelle un "capital social effervescent». Enfin, une troisième contribution de Mohamed Madoui éclaire le fonctionnement de réseaux ethniques familiaux et communautaires maghrébins soutenant l'installation comme petits entrepreneurs indépendants d'immigrés d'Afrique du Nord, en butte aux difficultés d'insertion professionnelle liées notamment à la discrimination. Un travail d'enquête qualitative conduit en Île-de-France et dans la région marseillaise a permis à l'auteur de mettre à jour les multiples «encastrements» familiaux, claniques et de réseaux qui structurent des relations d'entraide et de réciprocité basées sur la confiance et sur l'honneur; ces liens constituent un capital social qui soutient l'accès à l'entrepreneuriat et contribue à la coordination des activités économiques et à la circulation des biens.

\section{Capital social et fonctionnement démocratique}

Les trois dernières contributions de l'ouvrage, centrées sur l'apport du concept de capital social au fonctionnement démocratique des sociétés, ont une tonalité plus théorique. On ne sera pas étonné de trouver dans l'ouvrage une contribution consacrée au secteur de l'économie solidaire ou du tiers secteur, sous la plume de E. Buccolo et J.-L. Laville. Les auteurs y déclinent l'analyse du capital social que constituent les multiples formes de l'économie solidaire, organisées vers «la recherche de bénéfices collectifs », dans « un espace public de proximité», et mettent en avant sa dimension de «composante d'une démocratie forte», plutôt que de ressource essentiellement économique. Dans une contribution plutôt normative, le chercheur Vando Borghi fait appel au concept de capital social pour étudier les tensions entre processus de globalisation et dynamiques locales, d'encastrement local dans les territoires et de désencastrement des rapports sociaux liés aux changements d'échelle de la circulation de l'information, des capitaux, des biens et des 
personnes. Pour lui, le capital social est un «patrimoine local de réflexivité», constitué d'éléments concrets et abstraits tenant au vivre ensemble d'une société; ce patrimoine «fournit aux acteurs locaux les archétypes et les modèles de l'interaction coopérative», menacés par la globalisation et l'influence grandissante des entreprises et de la financiarisation. Ce capital social, dans une perspective politique, débouche sur l'émergence de formes d'un «agir public», où interagissent pratiques des individus et fonctionnement des institutions. Il rendra visibles les problèmes auxquels on s'affronte, permettra les apprentissages collectifs, sera guidé par le souci du bien collectif et poussera les communautés à ne pas se refermer sur elles-mêmes. La reconnaissance de la contribution de la sphère reproductive au capital social, en d'autres termes, de la prise en charge du caring work dans une société constitue aussi un aspect original de l'analyse de Vando Borghi, alors même que l'analyse en termes de rapports de genre n'est guère présente dans les travaux présentés dans cet ouvrage. On peut regretter par ailleurs que les auteurs n'aient pas évoqué au fil du texte quelques exemples donnant chair à leurs analyses.

\section{La dimension symbolique du monde social}

Bernard Perret, dans la dernière contribution de cette troisième partie, à la tonalité quasi-philosophique, s'interroge sur la capacité du concept de capital social à rendre compte des dynamiques macrosociales à l'œuvre dans une société, plus complexes que la seule dynamique associative. Il met en garde contre le risque de réduire le capital social à un instrument de mesure économique utilitariste qui ferait abstraction de la dimension symbolique de la constitution du «monde social». Mais il voit aussi la possibilité d'inclure dans la notion, qu'il interpréterait d'ailleurs plus en termes de patrimoine, la dimension politico-économique d'un développement durable: notion qui ferait place, à côté de la préservation de l'environnement pour les générations futures, à celle de la «durabilité de la société» comme préservation de «significations symboliques» et transmission «d'une capacité d'agir ensemble dans le registre du politique».

$\mathrm{Au}$ final, on retire de cet ouvrage une impression de foisonnement et de stimulation intellectuelle. Le lecteur, sociologue, politologue ou économiste, y trouvera un panorama étendu des horizons ouverts pour l'analyse sociologique par le «capital social», tant pour la comparaison internationale que pour l'étude des questions les plus classiques de la sociologie: il est également averti de ses limites et de la difficulté de son maniement. Mais au gré d'une sélection parmi les différentes contributions, l'ouvrage s'adresse à un plus large public: militants associatifs ou citoyens y trouveront matière à réflexion sur leurs engagements et sur la cohésion des collectifs ou sociétés auxquels ils participent.

\section{Le travail intenable. Résister collectivement à I'intensification du travail}

\author{
Sous la direction de Laurence \\ Théry(1)
}

La Découverte, Paris, 2006

Lu par Jean Bastien, Syndex (**)

L'intensification du travail prend des formes diverses selon les secteurs et selon les situations; elle n'en constitue pas moins une menace pour la santé de millions de travailleurs. En beaucoup d'endroits, les contraintes de productivité se cumulent en effet avec les contraintes exercées par les clients, auxquelles s'ajoutent désormais les contraintes psychosociales, liées aux modes de gestion et de management actuels, avec comme conséquence une forte dégradation des situations de travail. Dans ce contexte, et dans la foulée des enquêtes menées dans le cadre du chantier Le Travail en questions, la CFDT a décidé de faire de la santé au travail un axe de revendications nouvelles. Et elle a alors, parmi d'autres actions, entrepris de former des militants dans ce domaine. La méthode - originale - utilisée a consisté dans une formation à la recherche-action à laquelle ont participé une soixantaine d'entre eux, issus d'une vingtaine de sections syndicales d'entreprises ou d'administrations, qui ont été formés sur dix-huit mois en 2004-2005, encadrés par des formateurs syndicaux, des ergonomes, des médecins du travail et des sociologues. Elle a conduit les militants concernés à choisir un secteur de leur entreprise sur lequel mener l'enquête en allant interroger les salariés, pour partager ensuite avec eux les résultats de leurs analyses. Au passage, ils ont ainsi expérimenté une posture relativement nouvelle pour des représentants du personnel, privilégiant une élaboration collective du diagnostic et des revendications, elle-même basée sur une analyse de détail du travail, qui visait à prendre en compte la variabilité des conditions et le vécu des salariés. Ce livre rend compte de cette expérience originale de formation à travers la recherche-action.

\section{Des formes d'intensification du travail très diverses}

La première partie, rédigée par François Daniellou(2), donne à voir, à partir des situations analysées par les militants, les formes diverses que

(**) Syndex, cabinet d'expertise comptable spécialisé dans l'assistance aux comités d'entreprise.

(1) Inspectrice du travail, chargée de la santé au travail à la CFDT.

(2) Professeur d'ergonomie à l'université de Bordeaux, directeur du Laboratoire d'ergonomie des systèmes complexes. 
prend l'intensification du travail. Elle convoque ainsi successivement: la lingère qui assure, outre le repassage, la veille de nuit dans un foyer logement pour personnes âgées, le monteur sur chaîne automobile, soumis à des gestes répétitifs et au respect des cadences, l'ouvrière qui replace les biscuits sur une chaîne de conditionnement, le soudeur de pots d'échappement aux prises avec les méthodes d'organisation de production japonaises, la téléopératrice d'un centre d'appel qui n'en peut plus d'essayer de se conformer à tout ce qu'on lui demande, le conseiller de clientèle d'une compagnie d'assurance, spectateur malgré lui de la division du travail qui dégrade le service au client, la conductrice de ligne d'embouteillage, contrainte de jongler entre les trois lignes qu'elle est censée piloter en même temps, l'ouvrier de l'usine d'accessoires automobile qui fait le travail que l'automate n'arrive pas à faire, les fraiseurs de moules pour la verrerie qui ont dû renoncer à anticiper les bris d'outils et la non-qualité, les aides soignantes dans un service hospitalier de long séjour, confrontées à la situation des personnes dépendantes, les techniciennes d'intervention sociale et familiale qui peinent sur les rapports qu'on leur demande, l'employée de la société de nettoyage, gênée par les occupants et sanctionnée à cause de cela, les ouvriers sur une chaîne de découpe de volailles qui doivent s'accommoder du manque de place, et, pour finir, les ouvrières d'une exploitation viticole, auxquelles échoit le travail le moins qualifié.

C'est également l'occasion pour l'auteur de se pencher sur la situation faite aux femmes au travail (Laurence Théry y reviendra dans la seconde partie), qui, bien souvent, sous le poids des stéréotypes, concentre les causes d'intensification: le faible soutien du collectif, voire l'isolement au travail, la moindre autonomie, les contraintes et les pressions extérieures, le travail répétitif, dont les effets sur la santé sont particulièrement marqués. La situation des cadres n'est pas toujours meilleure, comme le note François Daniellou, pris entre le flux d'informations descendantes qu'ils doivent traduire et faire passer auprès de leurs subordonnés et le flux remontant du terrain et de la réalité quotidienne.

Dans tous les cas, «la flexibilité et la souplesse sont les maîtres-mots », que doivent permettre d'atteindre des réorganisations qui sont presque toujours «top-down», alors que la connaissance de la réalité $\mathrm{du}$ travail et la mobilisation de l'intelligence des salariés sont le plus souvent minimales. Dans ces conditions, faire ce qu'on leur demande de faire, sans leur mettre à disposition les moyens nécessaires et sans l'organisation adéquate, ne peut que constituer pour les salariés une source de frustration infinie, aux effets dommageables sur leur santé.

\section{Les pistes de transformation passent par la mise en discussion et l'implication des salariés}

La deuxième partie consiste dans une série de contributions de formateurs et de chercheurs qui présentent des éléments de connaissance ou de réflexion. Laurence Théry revient sur la démarche d'enquête approfondie et de restitution-discussion avec les salariés. La référence principale ici mobilisée est l'ergonomie, dans la mesure où c'est la mise en évidence de la variabilité des conditions du travail qui doit ouvrir des pistes de transformation. Mais la prise de conscience et la formulation collectives des problèmes sont tout aussi essentielles, dans la mesure où elles permettent de recréer du collectif et de donner de la voix à ces analyses, dans un domaine - 1'organisation du travail - que les directions ont tendance à considérer comme leur chasse gardée.

Bernard Dugué(3) se penche quant à lui, en sociologue et ergonome, sur les déterminants de l'intensification du travail. Celle-ci résulte le plus souvent, explique-t-il, d'une combinaison de décisions, faiblement coordonnées entre elles, généralement prises de surcroît sous l'influence d'une vision très péjorative de l'homme au travail, qui privilégie de fait des choix qui excluent tant la subjectivité des salariés que la réalité du travail, cela jusqu'aux systèmes de contrôle mis en place. Ce qui finit par démotiver complètement les salariés. À côté de cela, les changements sont systématiquement survalorisés par les directions; ils sont souvent mis en œuvre dans la précipitation et sans débat véritable concernant les choix effectués. «Ces évolutions, ces modes de fonctionnement et ces façons de conduire le changement doivent faire l'objet d'un regard critique. Il en va de la santé des entreprises comme de celle des salariés » (p. 113), explique l'auteur. Ce qui repose principalement sur la capacité des acteurs sociaux de l'entreprise 1) à interroger les projets pour chercher à favoriser la prise en compte des différentes logiques et la participation des salariés concernés et 2) à repérer et anticiper les effets des petites décisions, ce qui suppose qu'ils réussissent à dégager le temps nécessaire, mais aussi à retisser des liens avec les salariés (ce qui renvoie alors au travail de représentant du personnel, auquel l'auteur a consacré une thèse et un livre qui est paru l'an dernier chez Octarès) (4).

Corinne Gaudart(5) interroge quant à elle les évolutions du travail et leurs conséquences sur les travailleurs suivant leur âge et leur expérience (celles-ci prennent en effet une importance particulière dans la période de transition démographique et d'évolution technologique rapide que l'on connaît actuellement). L'empilement des contraintes se traduit par une pression temporelle croissante, or on sait que celle-ci est moins bien tolérée quand l'âge s'élève car elle réduit la possibilité pour les opérateurs de construire des compromis entre les exigences de la tâche et leurs capacités. Les coupures

(3) Docteur en sociologie et ergonome européen, chargé de recherches au Laboratoire d'ergonomie des systèmes complexes.

(4) Le travail de négociation - Regards sur la négociation collective d'entreprise, Octarès, 2005.

(5) Ergonome et chercheuse au CREAPT/CNRS. 
entre générations, liées notamment aux difficultés d'emploi, ont compromis les aides réciproques qui pouvaient exister entre classes d'âge. «Mais en nous désignant les dégâts qu'elle engendre, l'intensification nous montre aussi les moyens de lutter. Recréer des espaces de transmission dans l'entreprise permettrait de mettre du liant entre les âges et ainsi de débattre du sens du travail» (p. 137), conclut l'auteur.

La contribution de Philippe Davezies(6) creuse plus profond (on pourra également consulter les documents accessibles sur son site web). Comprendre le travail, explique-t-il, implique d'accorder un intérêt à l'activité, à la façon dont s'y construit le rapport aux objets et finalement à l'investissement subjectif de celui qui le réalise. Pour le faire bien, il faut sentir le travail, et donc payer de sa personne. Configurer des ressources, que l'on tire de soi (c'est ce qui confère à mon activité son style propre, mais également constitue fondamentalement ma capacité d'agir). Ce qui en retour contribue à former mon identité. C'est tout cela qu'un changement d'organisation peut alors mettre à mal, au risque de mettre en péril ma santé, comme on le comprend sans difficulté, quand celui-ci «tranche les liens sensibles qui, d'un côté, donnent chair à l'organisation du travail et, de l'autre, alimentent la vie psychique du sujet» (p. 148). Mais l'auteur va plus loin en expliquant qu'au-delà des objets sur lesquels j'investis, c'est l'activité d'autrui que je vise à travers mon travail, ou plus exactement mon articulation avec elle (même si cela n'exclut pas les conflits liés aux différences de perspectives). C'est ce qui explique du reste que le collectif ait alors une double fonction au regard du développement de l'activité, de défense de l'autonomie du métier contre les pressions qui tendent à le rabattre sur les seules dimensions instrumentales, et une fonction de transmission aux individus de l'expérience accumulée pour leur éviter les erreurs et leur permettre de progresser. On en tire également l'idée que pour transformer l'organisation, il faut procurer aux acteurs la possibilité de jouer un rôle actif dans la transformation. En attendant, «Ramener ainsi dans le débat social les conflits que les salariés portent comme des questions personnelles constitue un premier niveau de protection contre le sentiment de dévalorisation et contre les pathologies du stress » (p. 165), souligne 1'auteur, avant celui qui consisterait à transformer les situations.

\section{Interroger les pratiques syndicales?}

La troisième partie vise à faire le bilan de cette formation par la recherche-action, plutôt que des actions proprement dites menées par les équipes. On regrettera toutefois que les améliorations obtenues ne soient pas décrites, mais il était sans doute difficile d'en rendre compte, sauf à entrer dans un grand

(6) Enseignant et chercheur en médecine et santé au travail à l'université de Lyon 1. détail. Les deux premiers chapitres sont rédigés par de jeunes sociologues. Dans le premier, Cécile Guillaume (7) dresse les profils des militants et les conditions de leur engagement (la plupart sont élus de CHSCT), avant de se centrer sur les bénéfices individuels qu'ils ont pu en retirer. Une première catégorie de militants, eux-mêmes exposés à des situations de travail très difficiles, a vécu la formation, tout au moins la première étape, comme une sorte de thérapie individuelle, explique-t-elle, qui leur a permis de reconsidérer ce qu'ils vivaient sous l'éclairage d'une grille d'interprétation plus large. Malgré une certaine amélioration de leurs conditions de travail, ils «semblent aujourd'hui partagés entre un accablement devant l'ampleur du process d'intensification en marche - et le sentiment qu'il faut sans cesse recommencer le travail sur le plan syndical - et une prise de conscience de la nécessité de porter le débat à un niveau plus politique» (p. 183). Une seconde catégorie de militants, plus nombreux, moins directement concernés par l'intensification du travail, a mis à profit la formation pour renouer avec l'écoute des salariés et leur ressenti subjectif. Pour tous, l'expérience a permis la construction d'un sentiment d'appartenance plus fort à l'organisation. La plupart en ont profité pour se forger des compétences nouvelles en matière de santé au travail, et certains ont renforcé ainsi leur légitimité syndicale.

Le second chapitre, rédigé par Nadine Olivier(8), explore les fonctionnements collectifs. Pour la plupart des équipes, la démarche d'enquête n'a, au départ, pas été évidente, et il a souvent été difficile pour les militants de trouver la bonne distance. La rapidité d'évolution des structures d'entreprise a parfois compliqué les choses. En interne, l'équipe n'a pas toujours bénéficié du soutien du reste de la section syndicale (la répartition des rôles entre CHSCT et CE n'a pas facilité les choses). Mais les sections en question n'étaient pas non plus toutes dans la même situation en termes de poids ou d'influence dans l'entreprise. Finalement, les salariés ont été diversement associés à la restitution, même si certaines équipes ont réussi à impliquer les salariés tout au long du processus.

Le chapitre suivant, rédigé par Francis Bourdon et Danielle Mezzarobba, ergonomes et formateurs syndicaux, dresse enfin un bilan de l'expérience en tant que formation syndicale, soulignant tout son intérêt notamment pour permettre à des représentants du personnel de mieux saisir leur rôle et leur permettre de dépasser une vision a minima du travail syndical. Sur un sujet aussi complexe que la santé au travail, la démarche de recherche-action permet aux militants de comprendre tout l'intérêt d'alterner phases d'observation, de questionnement et de restitution pour une analyse objective de la situation et d'impliquer les salariés à la fois dans

(7) Sociologue, maître de conférence à l'université Lille 1.

(8) Sociologue, doctorante au Laboratoire interdisciplinaire pour la sociologie économique (CNRS/CNAM). 
le diagnostic et dans l'action collective destinée à remédier aux problèmes rencontrés. Ce qui appelle alors un travail collectif des militants et qui soit poursuivi sur la durée.

La conclusion revient à Laurence Théry, qui pointe notamment, après plusieurs auteurs, comme Marie-Anne Dujarier (9) ou d'autres regroupés autour de Yves Clot et Dominique L'Huillier (10), que «nous assistons aujourd'hui à une crise de l'organisation; et parce qu'on ne sait pas organiser, et parce que l'on fait fi de l'intelligence des salariés, les "organisateurs" reportent sur ces mêmes salariés la responsabilité de la réactivité, de la rapidité et le charge de la qualité. Certaines entreprises, certains services ou ateliers totalement désorganisés ne sont rentables que parce que les salariés sont mobilisés à $200 \%$ \% (pp. 227, 228), avec le risque d'atteintes graves à la santé que cela suppose. "Il est bon de le rappeler: le travail, ce n'est pas seulement l'aliénation. Il est bon d'être fier de son travail et, à l'inverse, il est douloureux de ne pas pouvoir le faire correctement. Le travail mobilise une bonne partie de notre vie, il engage notre identité et nous permet de nous réaliser: faire du bon travail, c'est d'abord trouver un espace où ce que l'on est va s'exprimer. C'est pouvoir renvoyer au collectif une expérience singulière qui n'est pas la simple répétition du travail des autres, mais une façon d'imprimer sa patte $[\ldots] »$ (p. 228). A contrario, «Quand on coince les gens dans des situations où tout est décidé sans eux, où tout est décidé au mépris de leur travail, alors apparaissent les atteintes à la santé. Nous en sommes là $[\ldots] »$ (p. 228).

On aura compris qu'il s'agit d'un livre engagé sur un sujet probablement appelé à connaître des développements importants au cours des prochaines années.

\section{Au risque de l'évaluation}

Salariés et candidats à l'emploi soumis aux aléas du jugement

\section{Marie-Christine Bureau et Emmanuelle Marchal (eds)}

\section{Presses universitaires du Septentrion}

\section{Lu par Hélène Garner(***)}

Ce livre rend compte d'un séminaire du Centre d'études de l'emploi organisé en 2003 autour de la question de l'évaluation du travail. L'objet de cet ouvrage est de mettre en exergue les enjeux de

(9) Marie-Anne Dujarier, L'idéal au travail, PUF, 2006; «La division sociale du travail d'organisation dans les services», Nouvelle revue de psychosociologie, no 1, 2006/1.

(10) Yves Clot, Dominique L'Huillier, «Perspectives en clinique du travail», Nouvelle revue de psychosociologie, no 1, 2006/1. $(* * *)$ Mission animation de la recherche - Dares. l'évaluation sur le marché du travail (des candidats à l'emploi mais aussi des salariés) dans un contexte dynamique d'évolution des compétences tout au long de la vie, mais aussi d'incertitude autour de la définition, et donc de l'évaluation, des compétences nécessaires pour occuper un poste.

Cet ouvrage collectif, composé de neuf contributions, est construit en deux parties; la première, intitulée «Les itinéraires de l'évaluation», met l'accent sur les modalités d'évaluation des personnes dans des contextes différents (en intérim et en entreprise) et selon des profils particuliers (jeunes et seniors). La seconde partie s'intéresse aux cadres de l'évaluation et analyse concrètement certaines pratiques d'évaluation; cette partie souligne la tendance à privilégier l'écrit dans l'évaluation et le renouvellement des pratiques d'évaluation en vigueur du fait des compétences nouvelles valorisées sur le marché du travail (celles relatives au comportement et à la personnalité des individus notamment) qui doivent être évaluées.

Le modèle d'évaluation actuel est caractérisé par une déconnexion à l'égard des situations de travail, par de nombreux outils disponibles dont la validité et la fiabilité restent à prouver, et la valorisation de qualités personnelles, relatives à la personnalité de l'évalué, dont l'évaluation est encore mal maîtrisée. Ces éléments concourent à accroître la subjectivité entourant l'évaluation et à renforcer les aléas du jugement; l'évaluation dans un contexte incertain est donc une opération risquée et périlleuse pour l'évalué comme pour l'évaluateur et nécessite une réflexion commune sur les modalités d'exercice. L'évaluation est risquée, car elle doit allier deux exigences difficilement compatibles: pouvoir prendre en compte les évolutions des compétences individuelles et assurer une relative stabilité du jugement à l'égard de la valeur de la personne.

L'approche multidisciplinaire proposée dans l'ouvrage (essentiellement économie et sociologie mais également ergonomie et psychologie) permet de «nourrir la réflexion sur les précautions à prendre pour éviter que ne se diffusent des pratiques génératrices d'exclusion et d'insécurité professionnelle». C'est un des enjeux de cet ouvrage: montrer que les pratiques d'évaluation actuelles sont susceptibles d'engendrer de l'exclusion sociale à l'encontre de certaines populations.

L'instabilité de l'emploi caractérisée par l'enchaînement de périodes d'emploi courtes conduit à une évaluation permanente des compétences des individus, qui peut être vécue comme une source d'insécurité supplémentaire. L'intérim est considéré comme représentatif de cette situation dans le sens où l'individu fait l'objet d'une évaluation permanente par l'entreprise de travail temporaire comme par les entreprises pour lesquelles il effectue des missions et qu'il se retrouve dans une situation de «mise à l'épreuve constante» (chapitre 1).

L'évaluation est un moment de tension car évaluateur et évalué sont dans l'incertitude, le premier est incertain quant à la qualité de son outillage et à la validité et la fiabilité de son jugement, et le second 
est incertain sur sa valeur personnelle et sur la capacité de l'évaluation subie à détecter ses compétences. Utilisée pour «situer» les individus les uns par rapport aux autres dans le système scolaire (chapitre 3), l'évaluation est un mode de sélection puis de management des salariés dans le milieu professionnel (chapitre 2) dont l'impact peut être source de stress et de mal-être au travail quand elle agit comme une sentence, ou valorisante quand elle s'adapte et intègre les caractéristiques des individus (cas des seniors dans les PME, chapitre 4).

Les alternatives proposées à ce modèle dans l'ouvrage et destinées à réduire les aléas du jugement consécutifs à l'évaluation tournent autour de deux idées: accroître la confiance entre évaluateurs et évalués afin de réduire l'incertitude sur la nature des compétences des évalués comme le permet la méthode IOD développée dans les structures d'insertion type mission locale (chapitre 6); «répartir» l'évaluation dans le temps mais aussi entre les individus afin de réduire l'arbitraire qui peut naître d'une évaluation individuelle.

Un autre enjeu de cet ouvrage est de faire de l'évaluation un objet d'étude en ce qu'il s'apparente à un jugement dont l'élaboration, qui oscille entre objectivité et subjectivité, mérite d'être approfondie; l'outillage utilisé pour évaluer les individus, le plus souvent sous couvert de recherche d'objectivité, s'apparente en réalité à de nouveaux modes de sélection et d'évaluation des individus toujours plus ou moins fondés sur l'arbitraire.
Plusieurs contributions dans l'ouvrage soulignent la tendance actuelle à la «reformalisation » des standards d'évaluation et à une montée en puissance de l'impact de l'écrit dans l'activité d'évaluation (chapitre 7). Deux exemples illustrent ces tendances; la graphologie constitue aujourd'hui, malgré les critiques sur sa faible valeur prédictive et sa fiabilité, un mode d'évaluation toujours utilisé pour évaluer la personnalité des candidats (chapitre 5). Le développement d'un marché de l'offre d'emploi sur internet favorise également un nouveau mode de codification et de filtrage des compétences demandées aux candidats qui «[...] conduit à valoriser les compétences les plus aisément codifiables $[\ldots] »$ ce qui n'est pas sans risque d'exclusion pour certaines personnes ne correspondant pas à ce profilage d'un type nouveau (chapitre 8).

Le dernier chapitre tente de répondre à la question capitale qui est celle des conditions d'une évaluation juste. Les grandes lignes d'une autorité du marché du travail qui serait garante de procédures justes d'évaluation sont esquissées (sur le modèle de l'Autorité de régulation des télécommunications). Fondée sur l'idée qu'une évaluation est d'autant plus juste qu'elle est plurielle (en termes d'intervenants comme d'outils) et soumise à une discussion publique, cette autorité serait composée de représentants d'entreprises, d'intermédiaires publics et privés du marché du travail, de salariés et de demandeurs d'emploi, d'acteurs associatifs et d'experts. 


\section{LABOUR MARKET}

\section{The emerging Sector of Web Sites Creation and the Scarcity and the low Visibility of Skills Yan Dalla Pria}

The objective of this article is to show that the scarcity and the low visibility of skills on the labour market of the emerging sector of web sites creation strongly influed on the functioning of the organisations specialised in this activity in France and in the United States in the late nineties. Relying on the case of a Parisian start-up called Babylone Connection, we propose to bring to light the richness and originality of the strategies developed by the pioneer start-up of this sector of activity regarding recruitment, employees loyalty and organisation of internal productive activities in order to adapt themselves to this very special situation of the labour market.

Keywords: labour market, skill, start-up, recruitment, control, carrier, staffing.

JEL: D210, M540, C800

\section{INTERNATIONAL}

\section{Employment Categories in Japanese Society}

Kurumi Sugita

This article analyses the employment categories used in the Japanese system of statistics. Through the analysis of questionnaires of principal labour force surveys, the author demonstrates the successive stratifications of signification in the course of history, which blur the contour of certain employment categories. More generally, the ambiguous character of employment categories seems to come from the adoption and utilisation in the statistic system of different forms of employment as expressed through ordinary denominations used in firms. The fact that the scientific classification system is based on a practice in the workplace suggests the important place occupied by the firm in Japanese society. However, an examination of the recent changes in the questionnaires reveals the tensions between the two different languages belonging to the fields of law, a very recent introduction, and of the firm. These tensions seem to increase in the present crisis where the State attempts to take up the functions abandoned by declining firms.

Keywords: Japan, employment, category, statistics

JEL: C190, J230, M510

\section{THEATRICAL FUSIONS IN EAST GERMANY}

\section{Laure de Verdalle}

In 1990, the differences between the theatrical landscapes of East Germany and West Germany are obvious. As a result, the consequences of the reunification onto the East German territory (where theatres are numerous and concentrated into small towns) are very strong. This restructuration process implies, among other things, the organisation of theatrical fusions that bring together different public institutions. These fusions straighten the rivalries between municipalities and cause a disorganization of the theatrical work. Through case analysis, I will show that this movement of concentration and rationalisation constitutes an interesting case to study the growing influence of management models in the cultural field. Because of the human and financial difficulties experienced through these fusions, I will also question the possibility of a feed-back impact of the reunification that could weaken the West German pattern of professional relationships.

Keywords: fusion, theatrical firms, West-Germany, Est.-Germany, professional relationships

JEL: D9120, J440, M500, L830 


\title{
EMPLOYMENT MARKET
}

\section{The relationship between innovation and employment in service}

\author{
Faridah Dajellal, Faïz Gallouj
}

This article is devoted to the difficult question of the relationship between innovation and employment. Its main objective is to re-examine the literature on innovation in services in the light of the employment issue. In particular, it attempts to assess to what extent and in what way this question is implicitly or explicitly addressed in the literature or deserves to be introduced into it. In pursuit of these objectives, the national and international literature is reviewed and a research agenda proposed. The following topics are explored in succession: technologist approaches and the employment question; the question of employment in service-based approaches.

Keywords: services, innovation, employment, scientific literature

JEL: M510, 0320, L840

\section{Public Employment Services contracting-out in France and the Australian, Dutch and British experiences}

\section{Céline Gratadour, Michèle Mansuy}

The contracting-out of reintegration services is a common trend among national Public Employment Services. It has been recently extended in France, under the guidance of both ANPE and Unédic. Australia and the Netherlands have gone the farthest towards contracting out all reintegration services. In the British Employment Zones, private providers are also contracted to take over long-term jobseekers completely. In order to enlighten the French present situation, this paper will explore the experiences of the three countries. First, we will consider which functions of the PES can be contracted out, and the way private providers are involved. In a second part, using the principal-agent theory, we will discuss the problems related to the privatised regimes, mainly a moral hazard problem. It consists in the private providers behaving opportunistically, while the government cannot perfectly observe their results. To overcome this problem, the government needs to use suitable governance mechanisms, ex ante (the new market has to be organised, the public employment agency needs to be reformed) and ex post (expected outcomes have to be clearly defined, incentive mechanisms to be employed, as well as information and control tools- as performance benchmarking and monitoring -).

Keywords: contracting out, reintegration services, unemployment, governance

JEL: H530, J680, 0570 


\section{TRAVAIL, GENRE ET SOCIÉTÉS}
L $\quad \mathbf{a}$
$r$ e $v$ u e
d u
M a g e

\section{$16 / 2006$}

HOMMAGE

NOVEMBRE 2006

\section{Madeleine Guilbert}

présenté par Margaret Maruani et Chantal Rogerat

\section{DOSSIER}

Les dégâts de la violence économique

Christian Trotzier

Isabelle Puech

Gao Yun, Florence Lévy et Véronique Poisson

\section{MUTATIONS}

Blanche Le Bihan-Youinou et Claude Martin

Stéphanie Gallioz

\section{RETOUR SUR...}

Colloque à Rabat

Danièle Meulders et Isabelle Puech

\section{CONTROVERSE}

Le "genre" interdit ?

Claudie Baudino, Huguette Dagenais,

Elisabeth Hofmann, Michel Bozon

\section{CRITIQUES}

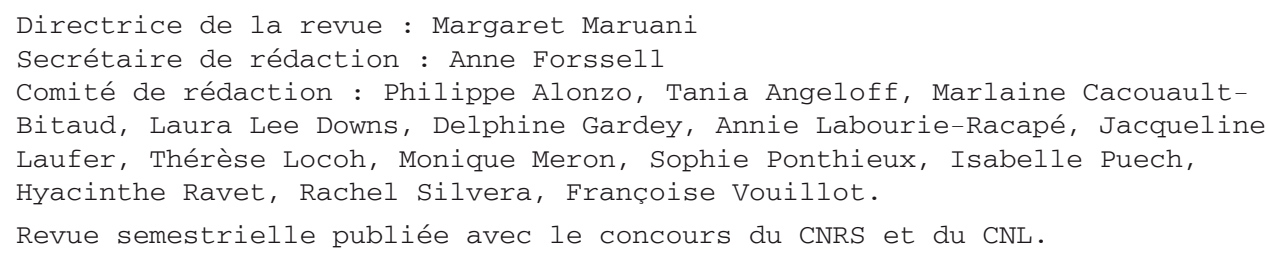

Abonnements :

Revues Armand Colin - Service Abonnements - 5, rue Laromiguière

75240 Paris cedex 05 - tel 33140648993

info@editions-sedes.com

http: //www.editions-sedes.com/ 
